\title{
Códigos cíclicos sobre anéis de cadeia
}

Anderson Tiago da Silva

\author{
TESE APRESENTADA \\ $\mathrm{AO}$ \\ Instituto De MatemÁticA E Estatística \\ DA \\ Universidade De SÃo Paulo \\ PARA \\ OBTENÇÃ̃O DO TÍTULO \\ DE \\ Doutor EM CIÊNCIAS
}

\author{
Programa: Matemática \\ Orientador: Prof. Dr. Francisco César Polcino Milies
}

Durante o desenvolvimento deste trabalho o autor recebeu auxílio financeiro da CAPES e do CNPq

São Paulo, 03 de fevereiro de 2012 


\section{Códigos cíclicos sobre anéis de cadeia}

Esta tese trata-se da versão original do aluno Anderson Tiago da Silva. 


\title{
Códigos Cíclicos sobre Anéis de Cadeia
}

\author{
Esta tese/dissertação contém as correções e alterações \\ sugeridas pela Comissão Julgadora durante a defesa \\ realizada por Anderson Tiago da Silva em 05/03/2012. \\ O original encontra-se disponível no Instituto de \\ Matemática e Estatística da Universidade de São Paulo.
}

Banca Examinadora:

- Prof. Dr. Francisco César Polcino Milies (orientador) - IME-USP

- Prof. Dr. Raul Antonio Ferraz - IME-USP

- Prof. Dr.Thierry Corrêa Petit Lobão - UFBA

- Profa. Dra. Sueli Irene Rodrigues Costa - UNICAMP

- Profa. Dra. Marinês Guerreiro - UFV 


\section{Agradecimentos}

Agradeço primeiramente a USP pela oportunidade e a CAPES e CNPq pelo suporte financeiro.

Aos membros da banca por aceitarem o convite, pelas dicas e correções.

A Deus, por ter me iluminado neste período da minha vida.

Aos meus pais, Marinalva e Rosino, pelo apoio nos momentos que mais precisei e por estarem sempre presentes, apesar da distância.

Aos meus irmãos, Alexsandro, Andreza e Andreia pela força.

Ao Robert pelo auxílio no programa MATLAB.

A minha mulher Daniela, pelos momentos felizes, pelo apoio, compreensão e pela paciência que teve comigo quando eu não estava bem.

Ao Prof. Dr. Francisco César Polcino Milies, que me orientou neste trabalho com paciência e me proporcionou grandes oportunidades acadêmicas durante o doutorado.

Aos professores do IME-USP, em especial ao prof. Dr. Raul Ferraz, por estar sempre disponível para me ouvir( sempre que eu descobria alguma coisa ia falar com ele antes de apresentar ao Polcino).

Aos amigos do IME, Alexander, John, Renata, Patricia, Fernanda, César e Ulisses, pelos momentos de descontração e pela companhia nos momentos de estudo.

A todos que contribuíram diretamente ou indiretamente com este trabalho. 


\section{Resumo}

\section{Códigos cíclicos sobre anéis de cadeia}

Neste trabalho, usamos uma abordagem de anéis de grupo para caracterizar códigos cíclicos sobre anéis de cadeia, seus duais e algumas condições sobre códigos auto-duais. Caracterizamos também os códigos cíclicos livres sobre anéis de cadeia e por fim exibimos uma fórmula para o peso de qualquer código cíclico sobre anéis de cadeia de comprimento $p^{n}$ e $2 p^{n}$.

Palavras-chave: anéis de grupo, anéis de cadeia, códigos cíclicos, peso de códigos cíclicos. 


\section{Abstract}

\section{Cyclic codes over chain rings}

In this thesis, we use an approach of group rings to characterize cyclic codes over chain rings, their duals and some conditions on self-dual codes. It also features free cyclic codes over chain rings and finally we show a formula for the weight of any cyclic code over chain rings of length $p^{n}$ and $2 p^{n}$.

Keywords: group rings, chain rings, cyclic codes, weight of cyclic codes. 


\section{Sumário}

1 Conceitos Preliminares $\quad 7$

1.1 Resultados sobre anéis e anéis de grupos . . . . . . . . . . . . . . . . 7

1.2 Códigos cíclicos . . . . . . . . . . . . . . . . . . . . . . . . . . . . . . . . . . . . . . . . . . .

1.3 Códigos cíclicos como ideais de $R G \ldots \ldots$. . . . . . . . . . . . . 16

1.4 Alguns Resultados sobre Resíduos Quadráticos . . . . . . . . . . . . . 19

2 Caracterização de Códigos Cíclicos sobre Anéis de Cadeia 23

2.1 Códigos Cíclicos sobre Anéis de Cadeia . . . . . . . . . . . . . . 24

2.2 Códigos duais e auto duais . . . . . . . . . . . . . . . . . . . . . . 29

3 Códigos de Comprimento $p^{n}$ sobre Anéis de Cadeia 37

3.1 Códigos que são livres como $R$-submódulos de $R G$. . . . . . . . . . . . 45

3.2 Códigos MDS de Comprimento $p^{n} \ldots \ldots$. . . . . . . . . . . . 50

3.3 Códigos MDS de Comprimento $2^{n} \ldots \ldots \ldots$. . . . . . . . . . . . 54

3.3.1 Resultados Sobre Códigos MDS de comprimento $p^{n}$. . . . . . . . 55

4 Códigos sobre Anéis de Cadeia de Comprimento $2 p^{n} \quad 57$

4.1 Peso Mínimo de Códigos de Comprimento $2 p^{n} \ldots$. . . . . . . . . . . 58

4.2 Códigos Livres de Comprimento $2 p^{n}$ Sobre Anéis de Cadeia . . . . . . . . . . 82

5 Conclusões Finais $\quad 85$

Referências Bibliográficas $\quad 89$

$\begin{array}{ll}\text { Bibliografia } & 91\end{array}$ 


\section{Introdução}

O avanço e a necessidade do uso de computadores e a utilização de qualquer aparelho ou atividade que envolve códigos tem levado ao crescente estudo de uma parte importante da Teoria da Informação, que é a Teoria dos Códigos Corretores de Erros, que lida com o problema geral da transmissão de mensagens de forma confiável. O marco inicial da teoria dos códigos corretores de erros é o trabalho de C.E. Shannon, "A Mathematical Theory of Communication", publicado em 1948.

A Teoria dos Códigos Corretores de Erros é um campo de pesquisa muito atual, tanto do ponto de vista científico quanto tecnológico. A junção da teoria e de suas aplicações vem tornando cada vez mais próximas a Matemática Pura e a Aplicada.

Descobertas recentes de que bons códigos binários não lineares estão relacionados com códigos lineares sobre $\mathbb{Z}_{4}$ (veja em [5], [8], [16], [30]) têm motivado os estudos de códigos sobre anéis em geral. Pesquisas mais recentes podem ser vistas em [9], [11], [20], [25].

Como uma extensão natural de $\mathbb{Z}_{4}$, em [6], Carlderbank e Sloane determinaram a estrutura de códigos cíclicos sobre $\mathbb{Z}_{p^{m}}$, depois, Kanwar e López-Permouth em [19] fizeram o mesmo, mas com diferentes demonstrações. Usando as mesmas técnicas que em [19], Wan em [37] estendeu os resultados de Kanwar e López-Permouth para códigos cíclicos sobre anéis de Galois. Em 1999, Norton e Sălăgean-Mandache em [28] estenderam os resultados 
de [6] e [19] para códigos cíclicos sobre anéis de cadeia finitos. Mais adiante, em 2004, Dinh e López-Permouth em [10], demonstraram os mesmos resultados de [28] de uma forma diferente.

O peso de um código é uma informação fundamental, juntamente com um método de decodificação. Em se tratando de códigos cíclicos sobre anéis, tanto o peso, quanto um método de decodificação são difíceis de determinar e isto vem sendo alvo de estudos na atualidade. Em [7] Campello, Jorge e Costa desenvolveram um método de decodificação de códigos q-ários utilizando a métrica de Lee. Vale ressaltar que códigos q-ários são exemplos de códigos cíclicos sobre anéis de cadeia. Em [2], Babu e Zimmermann exibem um algorítimo para decodificação de códigos sobre anéis de Galois, que também são exemplos de anéis de cadeia.

O objetivo principal deste trabalho é utilizar uma abordagem via anéis de grupo para provar de diferente forma os resultados de Dinh e López-Permouth em [10], onde as demonstrações de todos os resultados são feitas de forma mais simples e diretas e em alguns resultados, de forma mais geral. Como contribuição original, vamos determinar o peso de qualquer código cíclico sobre anéis de cadeia finitos de comprimento $p^{n}$ e $2 p^{n}$, com algumas hipóteses adicionais e iremos caracterizar também os códigos cíclicos livres sobre anéis de cadeia, exibindo duas bases distintas para qualquer somando de um código cíclico livre.

O Capítulo 1 consiste de uma revisão de conceitos preliminares de anéis, anéis de grupo, códigos corretores de erros, códigos cíclicos e uma conexão entre o estudo de ideais em álgebras de grupo e códigos cíclicos.

No Capítulo 2, usando uma abordagem de anéis de grupo, provamos os resultados de Dinh e López-Permouth [10] sobre códigos cíclicos sobre anéis de cadeia de comprimento $n$ e caracterizamos o código dual a um dado código e também os códigos auto-duais.

No Capítulo 3, consideramos o caso particular de códigos de comprimento $p^{n}$ com algumas hipóteses sobre a relação entre $q$ e $p^{n}$, onde $|R|=q^{l}$. Neste caso, a determinação dos códigos minimais depende unicamente da estrutura de subgrupos de $C_{p^{n}}$. Calculamos o peso de todos 
os possíveis códigos cíclicos sobre anéis de cadeia finitos (ou uniseriais), caracterizamos os códigos cíclicos livres sobre anéis de cadeia, exibindo duas bases distintas para um dado código e por fim exibimos alguns resultados para códigos MDS.

No Capítulo 4, restringindo o comprimento para $2 p^{n}$, calculamos o peso de todos os possíveis códigos cíclicos sobre anéis de cadeia e caracterizamos os códigos cíclicos livres sobre anéis de cadeia de forma análoga ao feito no Capítulo 3. 


\section{CAPÍTULO 1}

\section{Conceitos Preliminares}

Neste capítulo apresentaremos os conceitos preliminares necessários para o entendimento e boa compreensão do texto. Não será apresentada a demonstração dos resultados.

No que segue, estaremos sempre considerando anéis comutativos com unidade e assim, não faremos distinção entre ideais a direita e a esquerda tanto em definições quanto nos resultados.

\subsection{Resultados sobre anéis e anéis de grupos}

Os resultados nesta seção podem ser encontrados em [1], [18], [21], [26], [31]. Admitimos conhecidos os conceitos básicos da teoria de Anéis e listamos alguns deles para estabelecer a notação do trabalho.

Definição 1.1.1 Um anel $R$ é chamado anel de cadeia, ou uniserial, se o conjunto de todos os ideais formam uma cadeia com a relação de inclusão.

Definição 1.1.2 Seja $R$ um anel finito comutativo. Um ideal I de $R$ é chamado principal 
se ele é gerado por um único elemento. Um anel $R$ é chamado anel de ideais principais se todo ideal de $R$ é principal.

Definição 1.1.3 Um anel $R$ é chamado local se possui um único ideal maximal.

Teorema 1.1.4 Para um anel comutativo $R$ as seguintes condições são equivalentes:

(i) $R$ é um anel local e o ideal maximal $M$ de $R$ é principal.

(ii) $R$ é um anel local de ideais principais.

(iii) $R$ é um anel de cadeia.

Definição 1.1.5 Um anel $R$ é chamado anel serial, se $R$ é uma soma direta de anéis de cadeia.

Proposição 1.1.6 Sejam $R$ um anel de cadeia finito e comutativo com unidade, com ideal maximal $M=\langle a\rangle, t$ o indice de nilpotência de a em $R$ e $\bar{R}=\frac{R}{M}$. Então:

(a) Para algum primo $q$ e inteiros positivos $k$ e $l(k \geq l),|R|=q^{k},|\bar{R}|=q^{l}$ e a característica de $R$ e $\bar{R}$ são potências de $q$.

(b) Para $i=0,1,2, \ldots, t,\left|\left\langle a^{i}\right\rangle\right|=|\bar{R}|^{t-i}$. Em particular, $|R|=|\bar{R}|^{t}$, isto é, $k=l t$.

Definição 1.1.7 Um elemento e de um anel $R$ é dito idempotente se $e^{2}=e$. Dois idempotentes $e_{i}$ e $e_{j}$ são chamados ortogonais se $e_{i} \cdot e_{j}=0$. Um idempotente e é central se $e \cdot r=r \cdot e$, para todo $r \in R$. Um idempotente e é chamado primitivo se sempre que escrevermos $e=e_{1}+e_{2}$, com $e_{1}$ e $e_{2}$ idempotentes ortogonais, então ou $e_{1}=0$ ou $e_{2}=0$.

Proposição 1.1.8 Se $R=I_{0} \oplus \ldots \oplus I_{j}$ para alguns ideais $I_{0}, \ldots, I_{j}$, então existem idempotentes ortogonais $e_{0}, e_{1}, \ldots, e_{j}$ tais que $1=e_{0}+e_{1}+\ldots+e_{j}$ e $e_{k} R=I_{k}$, para cada $k \in\{0,1, \ldots, j\}$. 
Definição 1.1.9 Um ideal I de $R$ é chamado nil, se para cada $x \in I$, existe um inteiro $n$, tal que $x^{n}=0$.

Um ideal I de um anel $R$ é chamado nilpotente se existe um inteiro positivo $n$, tal que $I^{n}=0$, onde $I^{n}$ é o conjunto de todas as somas finitas da forma $\sum_{i=1}^{n} x_{1} x_{2} \ldots x_{n}$, com $x_{i} \in I, 1 \leq i \leq n$.

Definição 1.1.10 Seja $R$ um anel. O radical de Jacobson de $R$, denotado por $J(R)$ é a intersecção de todos os ideais maximais de $R$.

Proposição 1.1.11 Seja $R$ um anel. Todo ideal nil de $R$ está contido em $J(R)$.

É claro que se um ideal $I$ de $R$ é nilpotente, então $I$ é nil. Logo, todo ideal nilpotente esta contido em $J(R)$.

Proposição 1.1.12 ( [18], Proposição 7.14) Seja $R$ anel comutativo e $N$ um nil ideal em $R$, e $\bar{f}=u+N$ um idempotente de $\bar{R}=\frac{R}{N}$. Então existe um único idempotente e em $R$ tal que $\bar{e}=\bar{f}$.

Definição 1.1.13 Dois ideais $I_{1}$ e $I_{2}$ são chamados coprimos (ou comaximais) se $I_{1}+I_{2}=\langle 1\rangle$.

Claramente dois ideais $I_{1}$ e $I_{2}$ são coprimos se, e somente se, existem $x \in I_{1}$ e $y \in I_{2}$ tais que $x+y=1$.

Proposição 1.1.14 Se $I_{1}, \ldots, I_{n}$ são ideais coprimos aos pares, então

$$
I_{1} I_{2} \ldots I_{n}=I_{1} \cap I_{2} \cap \ldots \cap I_{n}
$$

Teorema 1.1.15 (Teorema Chinês do Resto) Se $I_{1}, \ldots, I_{n}$ são ideais de um anel $R$ coprimos dois a dois, então

$$
\frac{R}{I_{1} \ldots I_{n}} \simeq \frac{R}{I_{1}} \times \ldots \times \frac{R}{I_{n}}
$$


Definição 1.1.16 Seja $R$ um anel, $M$ um ideal de $R$ e $\bar{R}=\frac{R}{M}$. Considere a aplicação

$$
\begin{aligned}
-: R[x] & \rightarrow \bar{R}[x] \\
f(x)=\sum_{i=0}^{s} r_{i} x^{i} & \mapsto f(x)=\sum_{i=0}^{s} \bar{r}_{i} x^{i},
\end{aligned}
$$

onde $\bar{r}_{i}=r_{i}+M$, para todo $r_{i} \in R$. Um polinômio $f \in R[x]$ é chamado básico irredutivel sobre $R$ se $\bar{f}$ é irredutivel em $\bar{R}[x]$ e regular sobre $R$ se ele não é um divisor de zero.

Proposição 1.1.17 ( [21], Teorema XIII.2(c)) Seja $f=a_{0}+a_{1} x+\ldots+a_{n} x^{n} \in R[x]$. Então são equivalentes:

i) f é regular,

ii) $<a_{0}, \ldots, a_{n}>=R$,

iii) $a_{i}$ é uma unidade para algum $i, 0 \leq i \leq n$,

iv) $\bar{f} \neq 0$.

O próximo teorema é chamado de Lema de Hensel e pode ser encontrado em [21], Teorema XIII.4

Lema 1.1.18 (Lema de Hensel) Seja $f$ um polinômio sobre $R$ e assuma $\bar{f}=g_{1} g_{2} \ldots g_{r}$, onde $g_{1}, g_{2}, \ldots, g_{r}$ são polinômios coprimos dois a dois sobre $\bar{R}$. Então existem polinômios $f_{1}, f_{2}, \ldots, f_{r}$ coprimos dois a dois sobre $R$ tais que $f=f_{1} f_{2} \ldots f_{r}$ e $\bar{f}_{i}=g_{i}$, para $i=1,2, \ldots, r$. Proposição 1.1.19 ( [21], Teorema XIII.7) Seja $f$ um polinômio regular sobre $R[x]$. Se $f$ é básico irredutível, então $f$ é irredutível.

Definição 1.1.20 Seja $G$ um grupo e $R$ um anel. O anel de grupo de $G$ sobre $R$, denotado por $R G$, é o conjunto dos elementos da forma

$$
\alpha=\sum_{g \in G} x_{g} g
$$


onde $x_{g} \in R$ e $x_{g}=0$ quase sempre, ou seja, somente um número finito de coeficientes são diferentes de zero.

Definição 1.1.21 Dado um elemento $\alpha=\sum_{g \in G} a_{g} g \in R G$, definimos o suporte de $\alpha$, denotado por $\operatorname{supp}(\alpha)$ como sendo

$$
\operatorname{supp}(\alpha)=\left\{g \in G: a_{g} \neq 0\right\}
$$

Observe que, dados dois elementos $\alpha=\sum_{g \in G} a_{g} g$ e $\beta=\sum_{g \in G} b_{g} g \in R G$, temos $\alpha=\beta$ se, e somente se, $a_{g}=b_{g}$, para todo $g \in G$.

Com as seguintes definições de soma e produto de dois elementos em $R G$ e produto de um elemento de $R G$ por um elemento $\lambda \in R$, o anel de grupo $R G$ possui estrutura de anel e de $R$-módulo.

- $\left(\sum_{g \in G} a_{g} g\right)+\left(\sum_{g \in G} b_{g} g\right)=\sum_{g \in G}\left(a_{g}+b_{g}\right) g$

- $\alpha \cdot \beta=\sum_{g, h \in G} a_{g} b_{h} g h$, onde $\alpha=\sum_{g \in G} a_{g} g$ e $\beta=\sum_{h \in G} b_{h} h$;

- $\lambda\left(\sum_{g \in G} a_{g} g\right)=\sum_{g \in G} \lambda a_{g} g$

Definição 1.1.22 O homomorfismo

$$
\begin{aligned}
\xi: R G & \rightarrow \quad R \\
\left(\sum_{g \in G} a_{g} g\right) & \mapsto \sum_{g \in G} a_{g}
\end{aligned}
$$

é chamado aplicação de aumento de $R G$ e seu núcleo, denotado por $\triangle(G)=\left\{\sum_{g \in G} a_{g}(g-1): g \in G, g \neq 1\right\}$, é chamado ideal de aumento de $R G$.

Proposição 1.1.23 Se $H$ é um subgrupo de um grupo $G$, então

$$
\triangle(G: H)=\left\{\sum_{h t} \alpha_{h t} t(h-1): h \in H, h \neq 1, t \in \tau\right\}
$$


onde $\tau$ é um transversal de $H$ em $G$, é um ideal de $R G$.

Corolário 1.1.24 Se $H$ é um subgrupo normal de um grupo $G$, então

$$
\frac{R G}{\triangle(G: H)} \simeq R\left(\frac{G}{H}\right)
$$

Proposição 1.1.25 Se I é um ideal bilateral de um anel $R$ e $G$ é um grupo comutativo, então $I G=\left\{\sum_{g \in G} a_{g} g \in R G: a_{g} \in I\right\}$ é um ideal bilateral de $R G$ e $\frac{R G}{I G} \simeq\left(\frac{R}{I}\right) G$.

Proposição 1.1.26 Seja $\left\{R_{i}\right\}_{i \in I}$ uma família de anéis e seja $R=\oplus_{i \in I} R_{i}$. Então para qualquer grupo $G, R G \simeq \sum_{i \in I} R_{i} G$.

Teorema 1.1.27 (Teorema de Maschke) Seja G um grupo. O anel de grupo RG é semisimples se, e somente se, as seguintes condições são satisfeitas:

(i) $R$ é um anel semisimples.

(ii) G é finito.

(iii) $|G|$ é inversível em $R$.

Corolário 1.1.28 Sejam $G$ um grupo finito e $K$ um corpo. A álgebra $K G$ é semisimples se, e somente se, $\operatorname{car}(K)$ não divide $|G|$.

Corolário 1.1.29 Se $G$ é um grupo abeliano finito e $K$ um corpo tal que car $(K)$ não divide $|G|$, então $K G$ é uma soma direta de corpos.

Teorema 1.1.30 ( [21], Teorema VII.8) Seja e um idempotente não nulo de um anel $R$. As seguintes condições são equivalentes:

(1) e é primitivo.

(2) eRe é um anel local. 
(3) Re é indecomponível.

Note que, no caso particular em que $R$ e $G$ são comutativos, temos o seguinte.

Corolário 1.1.31 Seja e um idempotente não nulo de um anel RG. As seguintes condições são equivalentes:

(1) e é primitivo.

(2) RGe é um anel local.

(3) RGe é indecomponível.

Teorema 1.1.32 Sejam $R$ um anel com unidade e $H$ um subgrupo de um grupo $G$. Se $|H|$ é inversível em $R$, então $e_{H}=\widehat{H}$ é um idempotente em $R G$, onde $\widehat{H}=\frac{1}{|H|} \sum_{h \in H} h$. Além disso, se $H \triangleleft G$, então $e_{H}$ é central e

$$
R G e_{H} \simeq R\left(\frac{G}{H}\right)
$$

\subsection{Códigos cíclicos}

Nesta seção, abordaremos sem muitos detalhes conceitos de códigos corretores de erros. Os resultados e definições apresentados nesta seção podem ser encontrados em [6], [30].

Definição 1.2.1 Um conjunto finito A será chamado de alfabeto e o número de elementos de $A$ será denotado por $|A|$.

Definição 1.2.2 Um código corretor de erros é um subconjunto próprio qualquer de $A^{n}$, para algum número natural $n$. Os elementos de um código corretor de erros serão chamados de palavras.

A fim de tornar possível medir a distância entre palavras de um dado código em $A^{n}$, definiremos a seguir a distância de Hamming. 
Definição 1.2.3 Dados dois elementos $u=\left(u_{1}, \ldots, u_{n}\right)$ e $v=\left(v_{1}, \ldots, v_{n}\right) \in A^{n}$, a distância de Hamming entre $u$ e $v$ é definida por

$$
d(u, v)=\left|\left\{i ; u_{i} \neq v_{i}, 1 \leq i \leq n\right\}\right|
$$

Observe que a distância de Hamming é simétrica, satisfaz a desigualdade triangular e $d(u, v) \geq 0$, para todo $u, v \in A^{n}$. Por isso, é também chamada de métrica de Hamming.

Definição 1.2.4 A distância mínima de um código $C$ é o inteiro

$$
d(C):=\min \{d(x, y) ; x, y \in C \text { e } x \neq y,
$$

onde $d(x, y)$ é a distância de Hamming entre $x$ e $y$.

Uma classe de códigos muito utilizada na prática é a chamada classe dos códigos lineares. Para definir esta classe de códigos, iremos considerar um anel finito $R$ como sendo o alfabeto e $R^{n}$ um conjunto de $n$-uplas de elementos de $R$ como um módulo sobre $R$ de maneira usual.

Definição 1.2.5 Um subconjunto $C \subset R^{n}$ é chamado um código linear de comprimento $n$ sobre $R$, se $C$ é um $R$-submódulo próprio de $R^{n}$.

Definição 1.2.6 Dado $\beta=\left(r_{1}, \ldots, r_{n}\right) \in R^{n}$, defini-se o peso de $\beta$ como sendo o número inteiro

$$
w(\beta)=\left|\left\{i ; r_{i} \neq 0\right\}\right|
$$

Observe que o peso de um elemento $\beta$ é a distância de Hamming entre $\beta$ e zero.

Definição 1.2.7 O peso mínimo de um código linear $C \subset R^{n}$ é o inteiro

$$
w(C):=\min \{w(\beta) ; \beta \in C \backslash\{0\}\} .
$$

Proposição 1.2.8 Seja $C \subset R^{n}$ um código linear com distância d. Então, temos: 
(i) $d\left(\beta_{1}, \beta_{2}\right)=w\left(\beta_{1}-\beta_{2}\right)$, para todo $\beta_{1}, \beta_{2} \in R^{n}$.

(ii) $d(C)=w(C)$

Definição 1.2.9 Para um código linear $C$ de comprimento $n$ sobre $R$, definimos o posto de $C$, denotado por posto $(C)$, como sendo o número mínimo de geradores de $C$. Definimos o posto livre de $C$, denotado por postolivre $(C)$, como sendo o máximo dos postos de $R$ submódulos livres de $C$.

Definição 1.2.10 Dizemos que um código linear $C \subset R^{n}$ é livre se postolivre $(C)=$ $\operatorname{posto}(C)$.

Dentro da classe dos códigos lineares, existe uma importante subclasse de códigos, conhecida como classe dos códigos cíclicos, que será nosso principal interesse. Códigos cíclicos são importantes na prática, devido aos eficientes métodos de codificação e decodificação existentes quando tomamos o anel como sendo um corpo. Não entraremos em detalhes sobre codificação e decodificação, mas estes podem ser estudados em [14], onde o alfabeto em questão é um corpo.

Definição 1.2.11 Um código linear $C$ é dito cíclico se para toda palavra $\beta=\left(r_{1}, \ldots, r_{n}\right) \in$ $C$, sua troca cíclica também está em $C$, ou seja, a palavra $\beta^{\prime}=\left(r_{n}, r_{1}, \ldots, r_{n-1}\right) \in C$.

Sejam $u=\left(u_{1}, \ldots, u_{n}\right)$ e $v=\left(v_{1}, \ldots, v_{n}\right)$ elementos de $R^{n}$. Define-se o produto escalar de $u$ e $v$ por

$$
u \cdot v=u_{1} v_{1}+\ldots+u_{n} v_{n}
$$

O produto escalar possui as propriedades usuais de um produto interno.

Definição 1.2.12 Sejam $u$ e $v \in R^{n}$. Dizemos que $u$ e $v$ são ortogonais se $u \cdot v=0$.

Definição 1.2.13 Seja $C \subset R^{n}$ um código linear, define-se

$$
C^{\perp}=\left\{\beta \in R^{n} ; \beta \cdot u=0, \text { para todo } u \in C\right\} .
$$


Proposição 1.2.14 Se $C \subset R^{n}$ é um código linear, então $C^{\perp}$ é um $R$-submódulo de $R^{n}$.

Definição 1.2.15 Dado um código linear $C$, chamaremos de $C^{\perp}$ de código dual de $\boldsymbol{C}$.

Lema 1.2.16 Seja $R$ um anel finito de ordem $q^{\beta}$, $q$ primo. O número de palavras em qualquer código linear de comprimento $n$ sobre $R$ é $q^{k}$, para algum inteiro $k \in\{0, \ldots, \beta n\}$. Além disso, o código dual $C^{\perp}$ tem $q^{l}$ palavras, onde $k+l=\beta n$.

\subsection{Códigos cíclicos como ideais de $R G$}

Nesta seção exibiremos uma conexão entre códigos cíclicos e ideais em anéis de grupo. Os resultados aqui apresentados, podem ser encontrados em [24], [34].

Seja $R$ um anel comutativo com unidade e $G$ um grupo cíclico de ordem $n$ gerado por $g$. Seja $C$ um $R$-submódulo de $R^{n}$. Considere agora o homomorfismo de módulos $\psi$ dado por

$$
\begin{aligned}
\psi: R^{n} & \rightarrow R G \\
\beta=\left(r_{0}, \ldots, r_{n-1}\right) & \mapsto r_{0}+r_{1} g+r_{2} g^{2}+\ldots+r_{n-1} g^{n-1}
\end{aligned}
$$

Observe que $C$ é um código cíclico em $R^{n}$ se, e somente se, $\psi(C)$ é um ideal em $R G$. De fato, seja $C$ um código cíclico em $R^{n}$. Como $\psi$ é um $R$-homomorfismo, para todo $r \in R$ e $x \in C$, temos $r \cdot \psi(x)=\psi(r \cdot x)$. Como $C$ é um $R$-submódulo de $R^{n}$, temos $r \cdot x \in C$ e portanto $r \cdot \psi(x) \in \psi(C)$. Para provarmos que $\psi(C)$ é um ideal em $R G$ é suficiente provar que $g \cdot \psi(x) \in \psi(C)$, para todo $x \in C$. Seja $x=\left(x_{0}, \ldots, x_{n-1}\right) \in C$, $g \cdot \psi(x)=g \cdot\left(x_{0}+x_{1} g+\ldots+x_{n-1} g^{n-1}\right)=x_{n-1}+x_{0} g+x_{1} g^{2}+\ldots+x_{n-2} g^{n-1}$. Como $x=\left(x_{0}, \ldots, x_{n-1}\right) \in C$ e $C$ é cíclico, então $\left(x_{n-1}, x_{0}, \ldots, x_{n-2}\right) \in C$. Portanto,

$$
\begin{aligned}
\psi\left(\left(x_{n-1}, x_{0}, \ldots, x_{n-2}\right)\right) & =x_{n-1}+x_{0} g+x_{1} g^{2}+\ldots+x_{n-2} g^{n-1} \\
& =g \cdot \psi(x) \in \psi(C) .
\end{aligned}
$$

Agora suponhamos que $\psi(C)$ seja um ideal em $R G$. Devemos provar que $C$ é um código 
cíclico em $R^{n}$. Para isso, basta provar que se $x=\left(x_{0}, \ldots, x_{n-1}\right) \in C$, então $\left(x_{n-1}, x_{0}, \ldots, x_{n-2}\right) \in$ $C$. Como $x=\left(x_{0}, \ldots, x_{n-1}\right) \in C$, então $x_{0}+x_{1} g+\ldots+x_{n-1} g^{n-1} \in \psi(C)$. Como $\psi(C)$ é ideal, temos $g \cdot \psi(x) \in C . \quad \operatorname{Logo} x_{n-1}+x_{0} g+x_{1} g^{2}+\ldots+x_{n-2} g^{n-1} \in \psi(C)$. Assim, $\left(x_{n-1}, \ldots, x_{n-2}\right) \in C$.

Definição 1.3.1 A palavra $\left(a_{0}, a_{1}, \ldots, a_{n-1}\right) \in R^{n}$ é definida como palavra associada a $\alpha=a_{0}+a_{1} g+\ldots+a_{n-1} g^{n-1} \in R G$.

Uma outra abordagem muito utilizada no estudo de códigos é a de anéis polinomiais. É fácil ver que $R G \simeq \frac{R[x]}{\left(x^{n}-1\right)}$, onde $G$ é um grupo cíclico de ordem $n$ e $R$ anel comutativo com unidade.

Seja $x^{n}-1=\bar{g}_{0} \ldots \bar{g}_{m}$ a decomposição de $x^{n}-1$ em polinômios irredutíveis coprimos dois a dois em $\bar{R}[x]$. Pelo Lema de Hensel 1.1.18, sabemos que existem polinômios $f_{0}, \ldots, f_{m}$, coprimos dois a dois em $R[x]$ tais que $x^{n}-1=f_{0} \ldots f_{m}$. Como $f_{i}$ é regular, para $0 \leq i \leq m$, pela proposição 1.1.19, temos $f_{i}$ irredutível.

Sabemos que

$$
R G \simeq \frac{R[x]}{\left(x^{n}-1\right)}
$$

onde $G$ é um grupo cíclico de ordem $n$ e $x^{n}-1=f_{0} \cdot f_{1} \ldots f_{m}$ é a decomposição de $x^{n}-1$ como produto de polinômios irredutíveis coprimos dois a dois em $R[x]$. Pelo Teorema Chinês do Resto (1.1.15), temos

$$
\frac{R[x]}{\left(x^{n}-1\right)} \simeq \frac{R[x]}{\left(f_{0}\right)} \oplus \ldots \oplus \frac{R[x]}{\left(f_{m}\right)}
$$

Assim, informações importantes sobre os códigos cíclicos sobre $R$ podem ser obtidas através dos anéis $\frac{R[x]}{\left(f_{i}\right)}$. Observe também que devido ao isomorfismo podemos afirmar que existe um conjunto de idempotentes primitivos ortogonais com exatamente $m+1$ elementos, tal que

$$
R G=R G e_{0} \oplus \ldots \oplus R G e_{m}
$$


onde, reordenando as parcelas se preciso, temos $R G e_{r} \simeq \frac{R[x]}{\left(f_{r}\right)}$ e assim,

$$
\left|R G e_{r}\right|=|R|^{w_{r}} \text {, onde } w_{r}=\operatorname{grau}\left(f_{r}\right) .
$$

A fim de simplificar a notação, denotaremos $\frac{R[x]}{\left(x^{n}-1\right)}$ por $R_{n}$.

Teorema 1.3.2 Um polinômio mônico $p(x)$ em $R_{n}$ é um polinômio gerador para um código cíclico $C$, isto é, $C=<p(x)>$ se, e somente se, $p(x)$ divide $x^{n}-1$.

Teorema 1.3.3 Sejam $C_{1}=<g_{1}(x)>e C_{2}=<g_{2}(x)>$ códigos cíclicos em $R_{n}$. Então, $C_{1} \subset C_{2}$ se, e somente se, $g_{2}(x)$ divide $g_{1}(x)$.

Consideremos a seguinte caracterização para os idempotentes primitivos $e_{s}$ 's que pode ser encontrada em [34] ou [22]. Seja $C_{s}$ a classe ciclotômica módulo $n$ que contêm $s$ e $\alpha$ uma raiz $n$-ésima primitiva da unidade. Então,

$$
e_{s}=\frac{1}{n} \sum_{i=0}^{n-1} \sum_{j \in C_{s}} \alpha^{-i j} g^{i}
$$

Considere a aplicação

$$
\begin{array}{cl}
*: R G & \rightarrow R G \\
\alpha=a_{0}+a_{1} g+\ldots+a_{n-1} g^{n-1} & \mapsto \alpha^{*}=a_{0}+a_{1} g^{-1}+a_{2} g^{-2}+\ldots a_{n-1} g^{1}
\end{array},
$$

chamada involução clássica de $R G$. Vejamos algumas propriedades de *. Sejam $\alpha$, $\beta \in R G$ e $r \in R$. Temos

- $(\alpha+\beta)^{*}=\alpha^{*}+\beta^{*}$.

- $(r \cdot \alpha)^{*}=r(\alpha)^{*}$.

- $(\alpha \beta)^{*}=\beta^{*} \alpha^{*}=\alpha^{*} \beta^{*}$, pois $R G$ é comutativo. 
É fácil ver que * é um isomorfismo de anéis. Observe também que ${ }^{*}: R G e_{i} \rightarrow R G e_{i}^{*}$ é isomorfismo de anéis. Portanto, $\left|R G e_{i}\right|=\left|R G e_{i}^{*}\right|$.

Teorema 1.3.4 Sejam $\alpha=a_{0}+a_{1} g+\ldots+a_{n-1} g^{n-1}$ e $\beta=b_{0}+b_{1} g+\ldots+b_{n-1} g^{n-1}$. Então, $\alpha \cdot \beta=0$ em RG se, e somente se, $\left(a_{0}, a_{1}, \ldots, a_{n-1}\right)$ é ortogonal em $R^{n} a\left(b_{n-1}, b_{n-2}, \ldots, b_{1}, b_{0}\right)$ e todas as suas trocas cíclicas.

Definição 1.3.5 Dizemos que duas palavras $\alpha$ e $\beta \in R G$ são ortogonais se suas palavras associadas em $R^{n}$ são ortogonais.

\subsection{Alguns Resultados sobre Resíduos Quadráticos}

Nesta seção, apresentaremos alguns resultados sobre resíduos quadráticos, que serão necessários em resultados no próximo capítulo sobre códigos auto-duais. O conteúdo desta seção pode ser encontrado em [4], [27], [33], [35], [36].

Definição 1.4.1 Sejam a e $m$ inteiros relativamente primos, isto é, $\operatorname{mdc}(a, m)=1$. Se a congruência quadrática $x^{2} \equiv a(\operatorname{modm})$ tem uma solução, então a dito ser um resíduo quadrático de $m$, caso contrário a é chamado de não resíduo quadrático de $m$.

Euler deu um critério simples para saber quando um inteiro a é um resíduo quadrático de um dado primo $q$.

Teorema 1.4.2 Seja q um primo ímpar e $\operatorname{mdc}(a, q)=1$. Então a é um resíduo quadrático ou não resíduo quadrático de q quando $a^{\frac{q-1}{2}} \equiv 1(\operatorname{modq})$ ou $a^{\frac{q-1}{2}} \equiv-1(\operatorname{modq})$, respectivamente.

Para simplificar a notação, definiremos a seguir o símbolo de Legendre, introduzido pelo matemático Adrien Marie Legendre(1752-1833), em 1798. 
Definição 1.4.3 Seja q um primo ímpar e mdc(a,q)=1. O símbolo de Legendre $(a \mid q)$ é definido por:

$$
(a \mid q)=\left\{\begin{array}{c}
1, \quad \text { se a é um resíduo quadrático de } q . \\
-1, \quad \text { se a é um não resíduo quadrático de } q .
\end{array}\right.
$$

No símbolo de Legendre $(a \mid q)$, a e q são chamados numerador e denominador, respectivamente.

Observação 1.4.4 Pelo Teorema 1.4.2 e pela definição do símbolo de Legendre, para um inteiro impar q e um inteiro a com $\operatorname{mdc}(a, q)=1$, temos

$$
(a \mid q)=\left\{\begin{array}{c}
1, \quad \text { se e somente se, } a^{\frac{q-1}{2}} \equiv 1(\bmod q) . \\
-1, \quad \text { se e somente se, } a^{\frac{q-1}{2}} \equiv-1(\bmod q) .
\end{array}\right.
$$

A seguir, exibiremos algumas propriedades elementares do símbolo de Legendre.

Proposição 1.4.5 Sejam q um primo impar, a e b inteiros relativamente primos com $q$, Então:

(i) Se $a \equiv b(\bmod q)$, então $(a \mid q)=(b \mid q)$;

(ii) $(a \mid q) \equiv a^{\frac{q-1}{2}}(\bmod q)$;

$($ iii $)(a b \mid q)=(a \mid q)(b \mid q)$

(iv) $\left(a^{2} \mid q\right)=1$;

$(v)\left(a b^{2} \mid q\right)=(a \mid q)\left(b^{2} \mid q\right)=(a \mid q)$.

Teorema 1.4.6 (Lema de Gauss) Seja q um primo ímpar e mdc $(a, q)=1$. Se $k$ denota o número de inteiros no conjunto $S=\left\{a, 2 a, 3 a, \ldots, \frac{q-1}{2} a\right\}$ cujo resto da divisão por $q$ excede $\frac{q}{2}$, ent $\tilde{a} o(a \mid q)=(-1)^{k}$. 
Teorema 1.4.7 (Lei da Reciprocidade Quadrática) Se p e q são primos ímpares distintos, então

$$
(p \mid q)(q \mid p)=-1^{\left(\frac{p-1}{2} \frac{q-1}{2}\right)}
$$

Teorema 1.4.8 Se p e q são primos ímpares distintos, então

$(i)(p \mid q)(q \mid p)=\left\{\begin{array}{cc}1, & \text { se } p \equiv 1(\bmod 4) \text { ou } q \equiv 1(\bmod 4) \\ -1 & \text { se } p \equiv q \equiv 3(\bmod 4)\end{array}\right.$

$($ ii $)(p \mid q)=\left\{\begin{array}{cc}(q \mid p), & \text { se } p \equiv 1(\bmod 4) \text { ou } q \equiv 1(\bmod 4) \\ -(q \mid p) & \text { se } p \equiv q \equiv 3(\bmod 4)\end{array}\right.$

O Lema de Gauss 1.4.6 e a Lei da Reciprocidade Quadrática 1.4.7 nos permitem calcular $(a \mid q)$ para valores específicos de $a$. A proposição seguinte nos fornece uma lista de alguns destes cálculos.

Proposição 1.4.9 Seja q um primo ímpar. Então:

(i) $(1 \mid q)=1 e(-1 \mid q)=(-1)^{\frac{q-1}{2}}$;

(ii) $(-1 \mid q)=\left\{\begin{array}{cc}1, & \text { se } q \equiv 1(\bmod 4) \\ -1 & \text { se } q \equiv 3(\bmod 4)\end{array}\right.$

(iii) $(2 \mid q)=\left\{\begin{array}{c}1, \quad \text { se } q \equiv 1(\bmod 8) \text { ou } q \equiv 7(\bmod 8) \\ -1 \quad \text { se } q \equiv 3(\bmod 8) \text { ou } q \equiv 5(\bmod 8)\end{array}\right.$

$(i v)(-2 \mid q)=\left\{\begin{array}{c}1, \quad \text { se } q \equiv 1(\bmod 8) \text { ou } q \equiv 3(\bmod 8) \\ -1 \quad \text { se } q \equiv 5(\bmod 8) \text { ou } q \equiv 7(\bmod 8)\end{array}\right.$

$(v)(5 \mid q)=\left\{\begin{array}{c}1, \quad \text { se } q \equiv 1,9,11,19(\bmod 20) \\ -1 \quad \text { se } q \equiv 3,7,13,17(\bmod 20)\end{array}\right.$

$(v i)(6 \mid q)=\left\{\begin{array}{c}1, \quad \text { se } q \equiv 1,5,19,23(\bmod 24) \\ -1 \quad \text { se } q \equiv 7,11,13,17(\bmod 24)\end{array}\right.$ 
(vii) $(7 \mid q)=\left\{\begin{array}{r}1, \quad \text { se } q \equiv 1,3,9,19,25,27(\bmod 28) \\ -1 \quad \text { se } q \equiv 5,11,13,15,17,23(\bmod 28)\end{array}\right.$

(viii) $(11 \mid q)=\left\{\begin{array}{c}1, \quad \text { se } q \equiv 1,5,7,9,19,25,35,37,39,43(\bmod 44) \\ -1 \quad \text { se } q \equiv 3,13,15,17,21,23,27,29,31,41(\bmod 44) .\end{array}\right.$

Se além disso o primo ímpar q é $>3$, então,

$(i x)(3 \mid q)=\left\{\begin{array}{c}1, \quad \text { se } q \equiv 1(\bmod 12) \text { ou } q \equiv 11(\bmod 12) \\ -1 \quad \text { se } q \equiv 5(\bmod 12) \text { ou } q \equiv 7(\bmod 12) .\end{array}\right.$

$(x)(-3 \mid q)=\left\{\begin{aligned} 1, & \text { se } q \equiv 1(\bmod 6) \\ -1 & \text { se } q \equiv 5(\bmod 6)\end{aligned}\right.$

Proposição 1.4.10 Seja q um primo ímpar, $k$ um inteiro positivo e $\operatorname{mdc}(a, q)=1$. Então a é um resíduo quadrático de $q^{k}$ se, e somente se, $(a \mid q)=1$.

Teorema 1.4.11 Seja $m=2^{k_{0}} p_{1}^{k_{1}} \ldots p_{r}^{k_{r}}$ a fatorização de $m$, com $p_{i}$ primo e $m d c(a, m)=1$. Então a é um resíduo quadrático de $m$ se, e somente se,

(i) $\operatorname{para} i=1, \ldots, r,\left(a \mid p_{i}\right)=1$;

(ii) $a \equiv\left\{\begin{array}{cc}1(\bmod 4), & \text { se } 4 \mid m \text { mas } 8 \nmid m \\ 1(\bmod 8), & \text { se } 8 \mid \mathrm{m} .\end{array}\right.$ 


\section{CAPÍTULO 2}

\section{Caracterização de Códigos Cíclicos}

\section{sobre Anéis de Cadeia}

Neste capítulo iremos identificar todos os possíveis códigos cíclicos $C$ em anéis de grupo sobre um anel de cadeia finito $R$, comutativo com unidade, com $|R|=q^{k} \operatorname{com} q$ primo e $G$ um grupo cíclico finito de ordem $n$, onde $q$ não divide $n$. Determinaremos o número de códigos existentes sobre um dado anel, provaremos que $R G$ é um anel de ideais principal e para finalizar, iremos caracterizar o código dual a um dado código $C$ e também alguns resultados sobre códigos auto-duais.

Em [10], López-Permouth e Dinh caracterizam códigos sobre anéis de cadeia para um comprimento $n$ onde a característica do anel $R$ é $q^{m}$ e $q$ não divide $n$; determinam o número de elementos dos mesmo e exibem o dual de um dado código utilizando linguagem de anéis polinomiais. Aqui, faremos o mesmo utilizando uma abordagem de anéis de grupo, porém, de maneira mais simples. No próximo capítulo apresentaremos resultados novos, utilizando a abordagem de anéis de grupo. 


\subsection{Códigos Cíclicos sobre Anéis de Cadeia}

A seguir exibiremos alguns resultados para o desenvolvimento da teoria.

Observe que se $R$ é anel de cadeia comutativo com unidade e $M$ é o ideal maximal de $R$, então $\bar{R}=\frac{R}{M}$ é um corpo. Daqui em diante estaremos sempre nos referindo a anéis de cadeia onde $q \nmid|G|$.

Pela Proposição 1.1.6, sabemos que, $|R|=q^{k}$, então $\operatorname{car}(R)$ é uma potência de $q$.

A seguir, temos um teorema usado como ferramenta na caracterização dos códigos sobre anéis de cadeia, que é um caso particular da Proposição 1.1.25.

Teorema 2.1.1 Seja $R$ anel de cadeia e $M$ o ideal maximal de $R$. Então

$$
\frac{R G}{M G} \cong\left(\frac{R}{M}\right) G
$$

Como estamos considerando que $q \nmid n$, onde $|R|=q^{k}$ e $|G|=n$, pela Proposição 1.1.6, $\operatorname{car}\left(\frac{R}{M}\right) \nmid|G|$. Agora, pelo Corolário 1.1.28, $\left(\frac{R}{M}\right) G$ é semisimples e, pela Proposição 1.1.8, existem idempotentes primitivos ortogonais $\bar{e}_{0}, \ldots, \bar{e}_{m}$ tais que $\bar{R} G=\bar{R} G \bar{e}_{0} \oplus \ldots \oplus \bar{R} G \bar{e}_{m}$. Pelo Teorema 2.1.1 e pela Proposição 1.1.12, existe uma única família de idempotentes ortogonais $\left\{e_{0}, \ldots, e_{m}\right\}$ em $R G$ tal que $R G=R G e_{0} \oplus \ldots \oplus R G e_{m}$. Nosso objetivo agora é garantir que $\left\{e_{0}, \ldots, e_{m}\right\}$ é um conjunto de idempotentes primitivos ortogonais em $R G$.

Teorema 2.1.2 Sejam $R$ um anel local com ideal maximal $M=<a>$, com $|R|=q^{k} e$ $G$ um grupo cíclico de ordem $n$, onde $q \nmid n . S e\left\{\bar{e}_{o}, \ldots, \bar{e}_{m}\right\}$ é o conjunto de idempotentes primitivos ortogonais em $\bar{R} G$, então $\left\{e_{o}, \ldots, e_{m}\right\}$ é o conjunto de idempotentes primitivos ortogonais em $R G$.

Prova: Considere

$$
\begin{aligned}
& \phi: R G e_{k} \quad \rightarrow \quad \frac{R G}{M G} \bar{e}_{k} \\
& \sum_{i=0}^{n} r_{i} g^{i} e_{k} \mapsto \sum_{i=0}^{n} \bar{r}_{i} g^{i} \bar{e}_{k}
\end{aligned}
$$


Suponhamos $e_{k}=b_{k}+c_{k}$, onde $b_{k}$ e $c_{k}$ são idempotentes ortogonais. Então $\bar{e}_{k}=\bar{b}_{k}+\bar{c}_{k}$. Como $\bar{e}_{k}$ é um idempotente primitivo, temos $\bar{b}_{k}=0$ ou $\bar{c}_{k}=0$. Logo, $b_{k} \in M G$ ou $c_{k} \in M G$. Como $M G$ é nilpotente, temos $b_{k}=0$ ou $c_{k}=0$. Portanto, $e_{k}$ é idempotente primitivo.

No teorema a seguir, iremos caracterizar todos os códigos cíclicos de comprimento $n$ sobre $R G e_{i}$, onde $R$ é um anel de cadeia e $e_{i}$ é um idempotente primitivo ortogonal.

Sabemos pelo Corolário 1.1.31, que $R G e_{i}$ é um anel local. No que segue, para simplificar a notação, escreveremos os ideais da forma $(R G) a^{j} e_{i}$ como $\left\langle a^{j} e_{i}\right\rangle$.

Teorema 2.1.3 Seja $R$ um anel de cadeia finito, comutativo com unidade, com $|R|=q^{k}$, $M=\langle a\rangle$ ideal maximal de $R$ e $t$ o indice de nilpotência de a em $R$. Seja $G=C_{n}$, onde $q \nmid n$. Se I é um ideal de $R G e_{i}$, então I é da forma $I=\left\langle a^{k_{i}} e_{i}\right\rangle$, com $0 \leq k_{i} \leq t$.

Prova: Seja $I$ um ideal não nulo de $R G e_{i}$, com $I \neq(R G) e_{i}$. Seja $\zeta \neq 0$ um elemento de $I, \operatorname{logo} \zeta=x e_{i}, \operatorname{com} x \in R G$. Como $M$ é ideal maximal de $R,\left(\frac{R}{M}\right) G e_{i}$ é corpo e, como $\left(\frac{R}{M}\right) G e_{i} \simeq \frac{R G e_{i}}{M G e_{i}}$ e $\left(\frac{R}{M}\right) G e_{i}$ é uma componente simples de $\left(\frac{R}{M}\right) G$, pelo Corolário 1.1.29, $\frac{R G e_{i}}{M G e_{i}}$ é um corpo. Assim, concluímos que $M G e_{i}$ é ideal maximal de $R G e_{i}$. Pelo Corolário 1.1.31, sabemos que $R G e_{i}$ é anel local. Como $\zeta \in M G e_{i}$, podemos escrever $\zeta=\sum \alpha_{g} g e_{i}$, $\operatorname{com} \alpha_{g} \in M$. Então $\alpha_{g}=r_{g} a, r_{g} \in R$. Assim, $\zeta=\sum r_{g} a g e_{i}=\left(\sum r_{g} g\right) a e_{i} \in\left\langle a e_{i}\right\rangle$ e, portanto, $I \subset\left\langle a e_{i}\right\rangle$.

Agora seja $k$ o maior inteiro positivo tal que $I \subset\left\langle a^{k} e_{i}\right\rangle$. Com isso, existe $\zeta \in I$ tal que $\zeta$ não pertence a $\left\langle a^{k+1} e_{i}\right\rangle$. Provemos que $\zeta=a^{k} \beta e_{i}$, com $\beta \in R G e_{i}$ inversível. De fato, suponhamos que $\beta$ não seja inversível. Como $\left(\frac{R}{M}\right) G e_{i}$ é corpo, temos que $\beta \in M G e_{i}$; com isso, $\beta=a \beta^{\prime}, \beta^{\prime} \in R G e_{i}$ e assim temos $\zeta=a^{k} . a \beta^{\prime} e_{i} \in\left\langle a^{k+1} e_{i}\right\rangle$, o que é uma contradição. Logo, existe $\gamma \in R G e_{i}$ tal que $\beta \gamma=e_{i}$. Portanto, $a^{k} e_{i}=a^{k} \beta \gamma e_{i}=a^{k} \beta e_{i} \cdot \gamma e_{i}=(\zeta) \cdot \gamma e_{i} \in I$ e com isso concluímos que $I=\left\langle a^{k} e_{i}\right\rangle$. 
Corolário 2.1.4 Com as mesmas hipóteses do Teorema anterior, o ideal $R G e_{i}$ é indecomponível em $R G$ e o código gerado por $\left\langle a^{t-1} e_{i}\right\rangle$ é minimal.

Observe que como qualquer ideal $I$ de $R G e_{i}$ é da forma $I=\left\langle a^{k_{i}} e_{i}\right\rangle$, então $R G e_{i}$ é anel de cadeia.

Seja $\left\{e_{0}, \ldots, e_{m}\right\}$ o conjunto dos idempotentes primitivos de $R G$. No teorema a seguir iremos caracterizar todos os códigos cíclicos de comprimento $n$ sobre anéis de cadeia.

Teorema 2.1.5 Seja $R$ um anel de cadeia finito e comutativo, com ideal maximal $M=\langle a\rangle$, $t$ o indice de nilpotência de a em $R, \operatorname{com}|R|=q^{k}$ e $G=\left\langle g_{0} ; g_{0}^{n}=1\right\rangle$ tal que $q \nmid n$. Se $I$ é um ideal de $R G$, então $I$ é da forma $I=I_{0} \oplus \ldots \oplus I_{m}$, onde $I_{i}=\left\langle a^{k_{i}} e_{i}\right\rangle$, com $0 \leq k_{i} \leq t$.

Prova: Escrevemos $R G=R G e_{o} \oplus \ldots \oplus R G e_{m}$. Do Teorema 2.1.3, $R G e_{i}$ é um anel de cadeia. Falta provar apenas que se $I$ é um ideal de $R G$, então existem ideais $I_{0}, \ldots, I_{m}$ de $R G e_{0}, \ldots, R G e_{m}$ respectivamente, tais que $I=I_{0}+\ldots+I_{m}$. De fato, seja $I$ um ideal próprio não nulo de $R G$. Como $R G$ é finito, então $I$ é finito. Podemos listar os elementos de $I$ na forma $I=\left\{\alpha_{1}, \ldots, \alpha_{k}\right\}$. Como $\left\{e_{0}, \ldots, e_{m}\right\}$ é um conjunto de idempotentes ortogonais primitivos, com $1=e_{0}+\ldots+e_{m}$, temos :

$$
\begin{array}{ccc}
\alpha_{1}= & \alpha_{1} e_{0}+\ldots+\alpha_{1} e_{m} \\
\vdots & \vdots & \vdots \\
\alpha_{k} & = & \alpha_{k} e_{0}+\ldots+\alpha_{k} e_{m}
\end{array}
$$

Como $R G e_{0}$ é um anel de cadeia, existe $j_{0} \in\{1, \ldots, k\}$ tal que $\left\langle\alpha_{i} e_{0}\right\rangle \subset\left\langle\alpha_{j_{0}} e_{0}\right\rangle$, para todo $i \in\{1, \ldots, k\}$. Pelo Teorema 2.1.3, existe $k_{0}$ tal que $<\alpha_{j_{0}} e_{0}>=<a^{k_{0}} e_{0}>$. De forma análoga, para cada índice $\rho, 1 \leq \rho \leq m$, existe $j_{\rho}$ tal que $\left\langle\alpha_{i} e_{\rho}\right\rangle \subset\left\langle\alpha_{j_{\rho}} e_{\rho}\right\rangle$. Novamente pelo Teorema 2.1.3, existe $k_{\rho}$ tal que $\left\langle\alpha_{j_{\rho}} e_{\rho}\right\rangle=\left\langle a^{k_{\rho}} e_{\rho}\right\rangle=I_{\rho}$. Assim, dado $\alpha_{i} \in I$,

$$
\alpha_{i}=\alpha_{i} e_{0}+\ldots+\alpha_{i} e_{m} \in\left\langle a^{k_{0}} e_{0}\right\rangle \oplus \ldots \oplus\left\langle a^{k_{m}} e_{m}\right\rangle=I_{0} \oplus \ldots \oplus I_{m}
$$

Portanto $I \subset I_{0} \oplus \ldots \oplus I_{m}$. Segue imediatamente que $I_{0} \oplus \ldots \oplus I_{j} \subset I$. 
A seguir, iremos demonstrar um resultado que será necessário mais adiante.

Lema 2.1.6 Seja $R$ um anel local com ideal maximal $M=\langle a\rangle$ e $t$ o índice de nilpotência de a. Então $x \cdot a^{t-k}=0$ se, e somente se, $x \in\left\langle a^{k}\right\rangle$, onde $x \in R e 0<k<t$.

\section{Prova:}

Como $x$ não é inversível, temos que $x \in\langle a\rangle$. Seja $r<k$ o maior índice tal que $x \in\left\langle a^{r}\right\rangle$. Assim, $x=x_{1} \cdot a^{r}$. Mostremos que $x_{1}$ é inversível. De fato, se $x_{1}$ não é inversível, então $x_{1} \in\langle a\rangle$ e assim, $x \in\left\langle a^{r+1}\right\rangle$, o que é uma contradição. Como $x \cdot a^{t-k}=0$, temos que $x_{1} \cdot a^{t-k+r}=0$, onde $t-k+r<t$. Como $x_{1}$ é inversível, temos que $a^{t-k+r}=0$, o que contradiz o índice de nilpotência de $a$. Portanto, $x \in\left\langle a^{k}\right\rangle$.

Já vimos no capítulo anterior que

$$
R G=R G e_{0} \oplus \ldots \oplus R G e_{m} \simeq \frac{R[x]}{\left(x^{n}-1\right)} \simeq \frac{R[x]}{\left(f_{0}\right)} \oplus \ldots \oplus \frac{R[x]}{\left(f_{m}\right)}
$$

onde, reordenando as parcelas se necessário, temos $R G e_{i} \simeq \frac{R[x]}{\left(f_{i}\right)}$. Portanto, $\left|R G e_{i}\right|=|R|^{w_{i}}$, onde $w_{i}=\operatorname{grau}\left(f_{i}\right)$.

Teorema 2.1.7 Seja $C$ um código cíclico da forma $C=\left\langle a^{k_{i_{1}}} e_{i_{1}}\right\rangle \oplus \ldots \oplus\left\langle a^{k_{i_{r}}} e_{i_{r}}\right\rangle$. Então, o número de palavras de $C$ é $|C|=|\bar{R}|_{s=1}^{r}\left(t-k_{i_{s}}\right) w_{i_{s}}$.

Prova: Como $C$ é uma soma direta, temos $|C|=\left|\left\langle a^{k_{i_{1}}} e_{i_{1}}\right\rangle\right| \ldots\left|\left\langle a^{k_{i_{l}}} e_{i_{l}}\right\rangle\right|$. Devemos então determinar $\left|\left\langle a^{k_{i}} e_{i}\right\rangle\right|$.

Considere

$$
\begin{array}{cl}
\psi: R G & \rightarrow R G a^{k} . \\
\alpha & \mapsto \alpha a^{k}
\end{array}
$$


Claramente $\psi$ é um epimorfismo de grupos aditivos. O núcleo de $\psi$ é dado por

$$
\operatorname{ker}(\psi)=\left\{\alpha \in R G ; \alpha a^{k}=0\right\} .
$$

Pelo Lema 2.1.6, temos $\alpha a^{k}=0$ se, e somente se, $\alpha \in\left\langle a^{t-k}\right\rangle G$. Portanto,

$$
R G a^{k} \simeq \frac{R G}{\left\langle a^{t-k}\right\rangle G}
$$

Como $\frac{R G}{\left\langle a^{t-k}\right\rangle G} \simeq\left(\frac{R}{<a^{t-k}>}\right) G$, temos

$$
\left|R G a^{k_{i}} e_{i}\right|=\left|\left(\frac{R}{\left.<a^{t-k_{i}}\right\rangle}\right) G e_{i}\right|=\left|\frac{R}{\left\langle a^{\left.t-k_{i}\right\rangle}\right.}\right|^{w_{i}}=\left(\frac{|R|}{\left|\left\langle a^{t-k_{i}}\right\rangle\right|}\right)^{w_{i}}=|\bar{R}|^{\left(t-k_{i}\right) w_{i}} .
$$

$\operatorname{Logo,|C|=|\overline {R}|s=1} \sum_{s=1}^{r}\left(t-k_{i_{s}}\right) w_{i_{s}}$.

Seja $\left\{e_{0}, \ldots, e_{m}\right\}$ o conjunto dos idempotentes primitivos de $R G$. No teorema a seguir, iremos calcular o número de todos os códigos cíclicos possíveis sobre um anel de cadeia.

Teorema 2.1.8 Sejam $R$ um anel de cadeia finito e comutativo, com ideal maximal $M=<a>$, $t$ o indice de nilpotência de $a$, com $|R|=q^{k}$ e $G=<g_{0} ; g_{0}^{n}=1>$, onde $q \nmid n$. Então o número de códigos cíclicos de comprimento $n$ sobre $R$ é $(t+1)^{m+1}$.

Prova: Sabemos, do teorema anterior, que se $I$ é um ideal de $R G$, então $I$ é da forma $I=\left\langle a^{k_{0}} e_{0}\right\rangle \oplus \ldots \oplus\left\langle a^{k_{m}} e_{m}\right\rangle, \operatorname{com} 0 \leq k_{j} \leq t$.

Suponhamos $\left\langle a^{k} e_{i}\right\rangle=\left\langle a^{l} e_{i}\right\rangle$, com $k<l$. Assim, existe $\zeta \in R G$ tal que $a^{k} e_{i}=a^{l} e_{i} \zeta$. Multiplicando por $a^{t-l}$ em ambos os lados temos $a^{k+t-l} e_{i}=0$. Como $k<l$, temos que $k+t-l<t$ e, portanto, $a^{k+t-l} \neq 0$. Pelo Lema 2.1.6, $e_{i} \in M G$. Como $M G$ é nilpotente, existe $m$ tal que $e_{i}^{m}=0$. Como $e_{i}^{2}=e_{i}$, temos $e_{i}=0$, o que é uma contradição. Logo, $k=l$. Assim, para cada par de índice diferentes, teremos códigos diferentes, portanto o número de 
códigos será $(t+1)^{m+1}$.

Teorema 2.1.9 Para RG nas mesmas condições do Teorema 2.1.8, temos que RG é um anel de ideais principais.

Prova: Seja $I$ um ideal de $R G$. Então, pelo Teorema 2.1.5, $I=\left\langle a^{k_{0}} e_{0}\right\rangle+\ldots+\left\langle a^{k_{m}} e_{m}\right\rangle$.

É claro que $\left\langle a^{k_{0}} e_{0}+\ldots+a^{k_{m}} e_{m}\right\rangle \subset\left\langle a^{k_{0}} e_{0}\right\rangle+\ldots+\left\langle a^{k_{m}} e_{m}\right\rangle$. Provemos então que $\left\langle a^{k_{i}} e_{i}\right\rangle \subset\left\langle a^{k_{0}} e_{0}+\ldots+a^{k_{m}} e_{m}\right\rangle$. Seja $x \in\left\langle a^{k_{i}} e_{i}\right\rangle$, temos então que $x=\alpha a^{k_{i}} e_{i}$, onde $\alpha \in R G$. Como $e_{i} \cdot e_{k}=0$, para $i \neq k$ e $e_{i}^{2}=e_{i}$, temos $x=\alpha a^{k_{i}} e_{i}=\alpha e_{i}\left(a^{k_{0}} e_{0}+\ldots+a^{k_{m}} e_{m}\right)$.

Assim, $\left\langle a^{k_{i}} e_{i}\right\rangle \subset\left\langle a^{k_{0}} e_{0}+\ldots+a^{k_{m}} e_{m}\right\rangle$ e, portanto,

$$
\left\langle a^{k_{0}} e_{0}\right\rangle+\ldots+\left\langle a^{k_{m}} e_{m}\right\rangle=\left\langle a^{k_{0}} e_{0}+\ldots+a^{k_{m}} e_{m}\right\rangle
$$

Logo $R G$ é um anel de ideais principais.

\subsection{Códigos duais e auto duais}

Como vimos no capítulo anterior, os códigos duais de códigos cíclicos também são códigos cíclicos e nesta seção caracterizaremos os códigos duais de um dado código e os códigos auto duais.

Sejam $\left\{e_{0}, e_{1}, \ldots, e_{m}\right\}$ o conjunto dos idempotentes primitivos ortogonais de $R G$ tal que

$$
R G=R G e_{0} \oplus R G e_{1} \oplus \ldots \oplus R G e_{m}
$$

Pelo Teorema 2.1.7 temos que $\left|R G e_{i}\right|=|R|^{w_{i}}$. Como * denota a involução clássica, temos $\left|R G e_{i}\right|=\left|R G e_{i}^{*}\right|$. 
Proposição 2.2.1 Sejam $\alpha=a_{0}+a_{1} g+\ldots+a_{n-1} g^{n-1}$ e $\beta=b_{0}+b_{1} g+\ldots+b_{n-1} g^{n-1} \in R G$. Se $\alpha \cdot \beta^{*}=0$, então $\alpha$ é ortogonal a $\beta$.

Prova: Temos que $\beta^{*}=b_{0}+b_{n-1} g+b_{n-2} g^{2}+\ldots+b_{2} g^{n-2}+b_{1} g^{n-1}$. Como $\alpha \cdot \beta^{*}=0$, por 1.3.4, $\left(a_{0}, a_{1}, \ldots, a_{n-1}\right)$ é ortogonal a $\left(b_{1}, b_{2}, \ldots, b_{n-1}, b_{0}\right)$ em $R^{n}$ e todas as suas trocas cíclicas. Portanto, $\left(a_{0}, a_{1}, \ldots, a_{n-1}\right)$ é ortogonal a $\left(b_{0}, b_{1}, \ldots, b_{n-1}\right)$ e assim $\alpha$ é ortogonal a $\beta$.

- O resultado a seguir será necessário nos próximos teoremas.

Proposição 2.2.2 Seja $\left\{e_{0}, \ldots, e_{m}\right\}$ o conjunto dos idempotentes ortogonais, primitivos de RG. Então $\left\{e_{0}{ }^{*}, \ldots, e_{m}{ }^{*}\right\}$ é também o conjunto dos idempotentes ortogonais primitivos de $R G$.

Prova: Como ${ }^{*}: R G \rightarrow R G$ é um isomorfismo, segue que

(i) $e_{i}^{*} \cdot e_{i}^{*}=e_{i}^{*}$

(ii) $e_{i}^{*} \cdot e_{k}^{*}=0$, se $i \neq k$.

(iii) $1=e_{0}^{*}+\ldots+e_{m}^{*}$.

(iv) Cada $e_{i}^{*}$ é primitivo.

Consequentemente, $\left\{e_{0}^{*}, \ldots, e_{m}^{*}\right\}$ é o conjunto dos idempotentes primitivos de $R G$.

No próximo teorema iremos caracterizar o código dual a um dado código cíclico.

Teorema 2.2.3 Se um código cíclico $C$ é da forma $C=\left\langle a^{k_{0}} e_{0}\right\rangle \oplus \ldots \oplus\left\langle a^{k_{m}} e_{m}\right\rangle$, onde $0 \leq k_{i} \leq t$, então o código dual a $C$ é da forma $C^{\perp}=\oplus \sum_{r=0}^{m}\left\langle a^{t-k_{r}} e_{r}{ }^{*}\right\rangle$.

Prova: Seja

$$
\beta=\oplus \sum_{r=0}^{m}<a^{t-k_{r}} e_{r}^{*}>
$$

Assim,

$$
\left.|\beta|=|\bar{R}| \sum_{l=0}^{m} k_{l} w_{l}\right) .
$$


Como

$$
a^{k_{r}} e_{r} \cdot\left(a^{t-k_{s}} e_{s}^{*}\right)^{*}=0, \quad \forall 0 \leq r, s \leq m
$$

temos que $\beta \subset C^{\perp}$. Como $C=<a^{k_{0}} e_{0}>\oplus \ldots \oplus<a^{k_{m}} e_{m}>$, temos que

$$
|C|=|\bar{R}|^{m} \sum_{l=0}^{m}\left(t-k_{l}\right) w_{l}
$$

Suponha $|\bar{R}|=q^{\alpha}$. Assim, $|R|=q^{\alpha t}$.

Pelo Lema 1.2.16, temos $\left|C^{\perp}\right|=q^{l}$, onde

$$
\begin{aligned}
l & =\alpha t n-\alpha\left(\sum_{l=0}^{m}\left(t-k_{l}\right) w_{l}\right) \\
& =\alpha t n-\alpha \sum_{l=0}^{m}\left(t w_{l}\right)+\alpha \sum_{l=0}^{m} k_{l} w_{l} \\
& =\alpha t\left(n-\sum_{l=0}^{m} w_{l}\right)+\alpha \sum_{l=0}^{m} k_{l} w_{l} \\
& =\alpha\left(\sum_{l=0}^{m} k_{l} w_{l}\right) .
\end{aligned}
$$

Portanto, $\left|C^{\perp}\right|=|\beta|$ e $\operatorname{assim} C^{\perp}=\beta$.

Definição 2.2.4 Um código $C$ é chamado auto ortogonal se $C \subset C^{\perp}$ e auto-dual se $C=C^{\perp}$.

Nos teoremas a seguir, abordaremos os códigos auto-duais.

Agora seja $C=\left\langle a^{k_{0}} e_{0}\right\rangle \oplus \ldots \oplus\left\langle a^{k_{m}} e_{m}\right\rangle$.

Reordenando as parcelas se necessário, podemos escrever $C$ na forma

$$
C=\left\langle a^{r_{0}} e_{k_{01}}\right\rangle \oplus \ldots \oplus\left\langle a^{r_{0}} e_{k_{0 s_{0}}}\right\rangle \oplus \ldots \oplus\left\langle a^{r_{l}} e_{k_{l 1}}\right\rangle \oplus \ldots \oplus\left\langle a^{r_{l}} e_{k_{l s_{l}}}\right\rangle
$$

$\operatorname{com} 0 \leq r_{0}<r_{1}<\ldots<r_{l} \leq t$ 
Sejam

$$
\begin{gathered}
f_{0}=e_{k_{01}}+\ldots+e_{k_{0 s_{0}}} \\
f_{1}=e_{k_{11}}+\ldots+e_{k_{1 s_{1}}} \\
\vdots \\
f_{l}=e_{k_{l 1}}+\ldots+e_{k_{l_{l}}} .
\end{gathered}
$$

Temos $1=f_{0}+\ldots+f_{l}$. Além disso, $f_{i}$ e $f_{k}$ são idempotentes ortogonais, para $i \neq k$. Assim,

$$
C=\left\langle a^{r_{0}} f_{0}\right\rangle \oplus \ldots \oplus\left\langle a^{r_{l}} f_{l}\right\rangle
$$

Usando o Teorema 2.2.3 para o código descrito em 2.2, temos

$$
C^{\perp}=\left\langle a^{t-r_{l}} f_{l}^{*}\right\rangle \oplus \ldots \oplus\left\langle a^{t-r_{0}} f_{0}^{*}\right\rangle
$$

Definição 2.2.5 Para um anel de cadeia $R$, com ideal maximal $M=\langle a\rangle$, onde $t$ o índice de nilpotência de a é par, o código $C=\left\langle a^{\frac{t}{2}}\right\rangle$ é chamado código auto-dual trivial.

Proposição 2.2.6 Seja $C=\left\langle a^{r_{0}} f_{0}\right\rangle \oplus \ldots \oplus\left\langle a^{r_{l}} f_{l}\right\rangle$ um código cíclico. Então $C$ é auto-dual se, e somente se, para cada par de indices $i, j$ tais que $i+j \equiv 0(\bmod l)$ tem-se que $r_{i}+r_{j}=t$ $e f_{i}=f_{j}^{*}$.

Prova: Por 2.3, temos

$$
C^{\perp}=\left\langle a^{t-r_{l}} f_{l}^{*}\right\rangle \oplus \ldots \oplus\left\langle a^{t-r_{0}} f_{0}^{*}\right\rangle
$$

Note que o menor expoente de $a$ em $C^{\perp}$ é $t-r_{l}$. Assim, se $C=C^{\perp}$ temos que $r_{0}=t-r_{l}$, donde $r_{0}+r_{l}=t$ e $f_{0}=f_{l}^{*}$.

Da mesma forma, se $i+j \equiv 0(\bmod l)$, deve-se ter $r_{i}=t-r_{j}$, donde $r_{i}+r_{j}=t$ e $f_{i}=f_{j}^{*}$. Reciprocamente, se valem estas condições, é fácil ver que $C=C^{\perp}$.

Observação 2.2.7 Em [10], a notação usada por Dinh e López-Permouth exclui vários casos de códigos cíclicos auto-duais, pois usam a notação $C=\left\langle\widehat{F}_{1}, a \widehat{F}_{2}, \ldots, a^{t-1} \widehat{F}_{t}\right\rangle$ para o 
código, onde $x^{n}-1=F_{0} F_{1} \ldots F_{t}$. Aqui, enunciamos e provamos os teoremas para $C$ da forma $\left\langle a^{r_{0}} f_{0}\right\rangle \oplus \ldots \oplus\left\langle a^{r_{l}} f_{l}\right\rangle$, onde a única imposição sobre os expoentes $r_{i}$ 's de a é que $0 \leq r_{0}<r_{1}<\ldots<r_{l} \leq t$, o que ocorre para todos os possiveis ideais. Entraremos em mais detalhes no Capítulo 5.

Teorema 2.2.8 Suponha quet é um inteiro par. Então códigos cíclicos auto-duais diferentes do auto-dual trivial existem se, e somente se, existe um idempotente $e_{i} \in R G$, tal que $e_{i} \neq e_{i}^{*}$.

Prova: Vamos supor que $C=C^{\perp}$ é não trivial e que $e_{i}=e_{i}^{*}$, para todo índice $0 \leq i \leq l$. Reordenando os idempotentes, temos

$C=<a^{r_{0}} f_{0}>\oplus \ldots \oplus<a^{r_{l}} f_{l}>=<a^{t-r_{0}} f_{0}^{*}>\oplus \ldots \oplus<a^{t-r_{l}} f_{l}^{*}>=<a^{t-r_{0}} f_{0}>\oplus \ldots \oplus<a^{t-r_{l}} f_{l}>$

Logo, $r_{i}=t-r_{i}$, pata todo $0 \leq i \leq l$ e assim, $r_{i}=\frac{t}{2}$. Portanto, $C=<a^{\frac{t}{2}}\left(f_{0}+\ldots+f_{l}\right)>=$ $=<a^{\frac{t}{2}}>$, o que é uma contradição.

Reciprocamente, seja $e \in R G$ tal que $e \neq e^{*}$. Então $e^{*}$ é também um idempotente primitivo e podemos escrever $1=e+e^{*}+e_{3}+\ldots+e_{m}$. Denotemos $\beta=e_{3}+\ldots+e_{m}$. Assim, $1=e+e^{*}+\beta$. Por outro lado, $1=\left(e+e^{*}+\beta\right)^{*}=e^{*}+e+\beta^{*}$. Logo, $\beta=\beta^{*}$.

Considere

$$
C=<a^{\frac{t}{2}+1} e>\oplus<a^{\frac{t}{2}-1} e^{*}>\oplus<a^{\frac{t}{2}} \beta>
$$

Pelo Teorema 2.2.3 $C^{\perp}=<a^{\left(t-\left(\frac{t}{2}+1\right)\right)} e^{*}>\oplus<a^{t-\left(\frac{t}{2}-1\right)} e^{* *}>\oplus<a^{\frac{t}{2}} \beta^{*}>=C$. 
Teorema 2.2.9 Sejam $R$ anel de cadeia finito com ideal maximal $\langle a\rangle,|R|=q^{l t}$, onde $|\bar{R}|=q^{l}$ e $t$ o índice de nilpotência de a e seja $G$ um grupo cíclico de ordem $n$, onde $q \nmid n$. Se t é par, então códigos cíclicos auto-duais não triviais sobre $R$ existem se, e somente se, $q^{i} \not \equiv-1(\operatorname{modn})$ para todo inteiro positivo $i$.

Prova: Sabemos por 1.1 que um idempotente primitivo é da forma

$$
e_{s}=\frac{1}{n} \sum_{i=0}^{n-1} \sum_{j \in C_{s}} \alpha^{-i j} g^{i}
$$

Assim,

$$
e_{s}^{*}=\frac{1}{n} \sum_{i=0}^{n-1} \sum_{j \in C_{s}} \alpha^{i j} g^{i}=\frac{1}{n} \sum_{i=0}^{n-1} \sum_{j \in C_{n-s}} \alpha^{-i j} g^{i} .
$$

Pelo Teorema 2.2.8, códigos auto-duais não triviais existem se, e somente se, existe um idempotente $e_{k}$, tal que $e_{k} \neq e_{k}^{*}$. Agora, para todo idempotente $e_{k}, e_{k}=e_{k}^{*}$ se, e somente se, $C_{s}=C_{n-s}$. Portanto, para todo $0 \leq s \leq m, \Omega_{s}=\Omega_{n-s}$, onde $\Omega_{s}$ denota a $q$ classe ciclotômica contendo $s$. Isto acontece se, e somente se, existe $i$ tal que $q^{i} s \equiv(n-s)(\bmod n)$ para todo $0 \leq s \leq m$, ou equivalentemente $q^{i} \equiv-1(\bmod n)$

Corolário 2.2.10 Se n é um número primo, então códigos cíclicos auto-duais de comprimento $n$ ñ̃o existem nos seguintes casos:

- $q=2, n \equiv 3,5(\bmod 8)$;

- $q=3, n \equiv 5,7(\bmod 12)$;

- $q=5, n \equiv 3,7,13,17(\bmod 20)$;

- $q=7, n \equiv 5,11,13,15,17,23(\bmod 28)$;

- $q=11, n \equiv 3,13,15,17,21,23,27,29,31,41(\bmod 44)$

Prova: Segue do Teorema 2.2.9, Observação1.4.4 e Proposição1.4.9. 
Corolário 2.2.11 Seja n um primo ímpar diferente de q, e q um não resíduo quadrático de $n^{k}$, onde $k \geq 1$. Então códigos cíclicos auto-duais de comprimento $n$ não existem.

Prova: Segue dos Teorema2.2.9, Observação1.4.4 e Proposição1.4.10.

Corolário 2.2.12 Se n é um primo ímpar, então códigos cíclicos auto-duais de comprimento $n$ não existem nos seguintes casos:

- $q \equiv 1(\bmod 4)$ e existe um inteiro $k$ tal que $\operatorname{mdc}\left(q, 4 n^{k}\right)=1$ e q é um não resíduo quadrático de $4 n^{k}$;

- $q \equiv 1(\bmod 8)$ e existem inteiros positivos $i, j$ tais que $i>2, \operatorname{mdc}\left(q, 2^{i} n^{j}\right)=1$ e q é um não resíduo quadrático de $2^{i} n^{j}$.

Prova: Segue do Teorema2.2.9, Observação1.4.4 e Proposição1.4.11. 


\section{CAPÍTULO 3}

\section{Códigos de Comprimento $p^{n}$ sobre}

\section{Anéis de Cadeia}

Neste capítulo vamos considerar o caso particular de códigos cíclicos de comprimento $p^{n}, \operatorname{com} p$ primo, sobre um anel de cadeia $R$, com ideal maximal $M=<a>$ tal que $|\bar{R}|=q^{l}$, $\operatorname{com} o(|\bar{R}|)=\phi\left(p^{n}\right)$ em $U\left(\mathbb{Z}_{p^{n}}\right)$ e $q \nmid p^{n}$. Neste caso, Ferraz e Polcino Milies em [15] provaram que os idempotentes primitivos de $\bar{R} G$ dependem unicamente da estrutura de subgrupos de $C_{p^{n}}$ e deram sua fórmula explícita.

Para um grupo cíclico $G$ de ordem $p^{n}$, o reticulado de subgrupos de $G$ formam uma cadeia:

$$
G=G_{o} \supset G_{1} \supset \ldots \supset G_{n}=1 .
$$

Neste caso, os elementos

$$
e_{0}=\widehat{G} \quad \text { e } \quad e_{i}=\widehat{G}_{i}-\widehat{G}_{i-1}, \text { para } 1 \leq i \leq n,
$$


formam o conjunto dos idempotentes ortogonais tal que

$$
e_{0}+e_{1}+\ldots+e_{n}=1
$$

Se $|\bar{R}|=q$ e $o(q)=\varphi\left(p^{n}\right)$ em $U\left(\mathbb{Z}_{p^{n}}\right)$, onde $\phi$ denota a função de Euler, temos o seguinte.

Teorema 3.0.13 ( [15], Teorema 3.1 ) Seja F um corpo com q elementos e G um grupo cíclico de ordem $p^{n}$ tal que o(q)= $\phi\left(p^{n}\right)$ em $U\left(\mathbb{Z}_{p^{n}}\right)$. Seja

$$
G=G_{0} \supset G_{1} \supset \ldots \supset G_{n}=\{1\}
$$

a cadeia descendente de todos os subgrupos de G. Então o conjunto dos idempotentes primitivos de FG é dado por

$$
\bar{e}_{o}=\frac{1}{p^{n}}\left(\sum_{g \in G} g\right) \quad e \quad \bar{e}_{i}=\widehat{G}_{i}-\widehat{G}_{i-1}, \quad 1 \leq i \leq n .
$$

A seguir, iremos estabelecer uma hipótese central que consideraremos ao longo do capítulo.

\section{Hipótese A}

Sejam $R$ anel de cadeia finito, comutativo com unidade, tal que $|R|=q^{k}, M=<a>$ o ideal maximal de $R,|\bar{R}|=q^{l}$, com $k=l t$, onde $t$ é o indice de nilpotência de a e $G$ um grupo cíclico de ordem $p^{n}$ com gerador $g_{0}$, tal que o $(|\bar{R}|)=\phi\left(p^{n}\right)$ em $U\left(\mathbb{Z}_{p^{n}}\right), G_{i}=<g_{0}^{p^{i}}>$ $e e_{i}=\widehat{G}_{i}-\widehat{G}_{i-1}$.

Daqui em diante, estaremos sempre nas condições da Hipótese A.

Pelo Teorema 3.0.13, $\overline{e_{0}}=\widehat{G}, \overline{e_{i}}=\widehat{G_{i}}-\widehat{G_{i-1}}$ formam o conjunto dos idempotentes primitivos ortogonais de $\left(\frac{R}{<a>}\right) G$. Pelo levantamento, $e_{0}=\widehat{G}$ e $e_{i}=\widehat{G_{i}}-\widehat{G_{i-1}}$ formam o conjunto dos idempotentes primitivos ortogonais de $R G$. 
Para este conjunto de idempotentes primitivos, o próximo teorema exibe uma fórmula do número de elementos nos códigos da forma $I=<a^{k_{0}} e_{0}>\oplus \ldots \oplus<a^{k_{n}} e_{n}>$.

Teorema 3.0.14 Para $R$ e $G$ nas condições da Hipótese A, se I é um ideal de RG da forma $I=<a^{k_{0}} e_{0}>\oplus \ldots \oplus<a^{k_{m}} e_{m}>$, com $0 \leq k_{i} \leq t$, ent $\tilde{a} O$

$$
|I|=|\bar{R}|^{\left[\sum_{j=1}^{m}\left(t-k_{j}\right)\left(p^{j}-p^{j-1}\right)+\left(t-k_{0}\right)\right]}
$$

Prova: Como $C$ é uma soma direta, temos $|C|=\left|<a^{k_{0}} e_{0}>\right| \ldots\left|<a^{k_{m}} e_{m}>\right|$. Devemos então determinar $\left|<a^{k_{i}} e_{i}>\right|$. Seja $i>0$, temos que $a^{k_{i}} e_{i}=a^{k_{i}} \widehat{G}_{i}-a^{k_{i}} \widehat{G}_{i-1}$, com $e_{i}+\widehat{G}_{i-1}=\widehat{G}_{i}$ e $e_{i} \cdot \widehat{G}_{i-1}=\left(\widehat{G}_{i}-\widehat{G}_{i-1}\right) \cdot \widehat{G}_{i-1}=0 . \log \mathrm{O}$

$$
R G a^{k_{i}} \widehat{G}_{i}=R G a^{k_{i}} e_{i} \oplus R G a^{k_{i}} \widehat{G}_{i-1}
$$

Assim, $\left|R G a^{k_{i}} e_{i}\right|=\frac{\left|R G a^{k_{i}} \widehat{G}_{i}\right|}{\left|R G a^{k_{i}} \widehat{G}_{i-1}\right|}$.

Considere

$$
\begin{array}{cc}
\psi: R G & \rightarrow R G a^{k_{i}} . \\
\alpha & \mapsto \alpha a^{k_{i}}
\end{array}
$$

Claramente $\psi$ é um epimorfismo de grupos aditivos. O núcleo de $\psi$ é dado por

$$
\operatorname{ker}(\psi)=\left\{\alpha \in R G ; \alpha a^{k_{i}}=0\right\}
$$

Pelo Lema 2.1.6, temos $\alpha a^{k_{i}}=0$ se, e somente se, $\alpha \in<a^{t-k_{i}}>G$. Portanto,

$$
R G a^{k_{i}} \simeq \frac{R G}{<a^{t-k_{i}}>G}
$$


Como $R G /<a^{t-k_{i}}>G \simeq\left(R /<a^{t-k_{i}}>\right) G$, temos

$$
\left|R G a^{k_{i}} \widehat{G}_{i}\right|=\left|\left(\frac{R}{<a^{t-k_{i}}>}\right) G \widehat{G}_{i}\right|
$$

Denotemos $R /<a^{t-k_{i}}>$ por $\bar{R}_{k_{i}}$. Lembremos que $\left|\bar{R}_{k_{i}}\right|=\frac{|\bar{R}|^{t}}{|\bar{R}|^{k_{i}}}$.

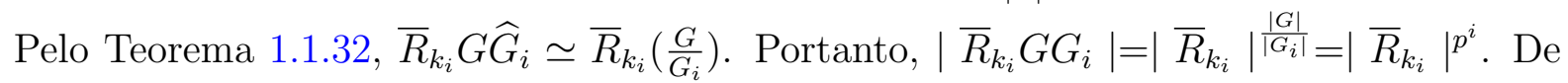
forma análoga, $\left|R G a^{k_{i}} \widehat{G}_{i-1}\right|=\left|\bar{R}_{k_{i}}\right|^{p^{i-1}}$. Logo,

$$
\left|R G a^{k_{i}} e_{i}\right|=\left(\frac{|\bar{R}|^{t}}{|\bar{R}|^{k_{i}}}\right)^{p^{i}-p^{i-1}}=|\bar{R}|^{\left(t-k_{i}\right)\left(p^{i}-p^{i-1}\right)} .
$$

Para finalizar, calculemos $\left|<a^{k_{0}} e_{0}>\right|$. Pelo Lema 3.1, temos

$$
\left|R G a^{k_{0}} e_{0}\right|=\left|\left(\frac{R}{<a^{t-k_{0}}>}\right) G \widehat{G}\right|
$$

Logo,

$$
\left|R G a^{k_{0}} e_{0}\right|=\left(\frac{|R|}{<a^{t-k_{0}}>}\right)^{\frac{|G|}{|G|}}=\frac{|\bar{R}|^{t}}{|\bar{R}|^{k_{0}}}=|\bar{R}|^{\left(t-k_{0}\right)} .
$$

Portanto, $|I|=|\bar{R}|^{\left[\sum_{j=1}^{m}\left(t-k_{j}\right)\left(p^{j}-p^{j-1}\right)+\left(t-k_{0}\right)\right]}$.

Tão importante quanto descrever todos os possíveis códigos cíclicos é saber o seu peso mínimo. Nos teoremas que seguem, determinaremos o peso mínimo de todos os códigos cíclicos nas condições das hipóteses anteriores. Os resultados seguintes são uma generalização de resultados semelhantes obtidos por F. Mello [23] para corpos, à situação que estamos considerando.

Teorema 3.0.15 Considere $R \quad$ e $G$ nas condições da Hipótese A. Então $w\left((R G) a^{k} e_{i}\right)=2\left|G_{i}\right|$, para $i \neq 0$ e $\left.w\left((R G) a^{k} e_{0}\right)\right)=|G|$, para $0 \leq k \leq t-1$. 


\section{Prova:}

Para $i \neq 0$, temos $e_{i} \widehat{G}_{i}=\left(\widehat{G}_{i}-\widehat{G}_{i-1}\right) \widehat{G}_{i}=\widehat{G}_{i}-\widehat{G}_{i-1}=e_{i} . \operatorname{Logo},(R G) e_{i} \subset(R G) \widehat{G}_{i}$. Seja $\Gamma$ um transversal de $G_{i}$ em $G$. Um elemento arbitrário $\alpha \in R G$ pode ser escrito na forma $\alpha=\left(\sum_{h \in \Gamma} \alpha_{h} h\right)$, com $\alpha_{h} \in R G_{i}$. Portanto, qualquer palavra sobre $(R G) \widehat{G}_{i}$ é da forma $\alpha=\left(\sum_{h \in \Gamma} x_{h} h\right) \widehat{G}_{i}, x_{h} \in R$.

Como $(R G) a^{k} e_{i} \subset(R G) a^{k} \widehat{G}_{i}$ qualquer palavra de $(R G) a^{k} e_{i}$ também é da forma $\alpha=\left(\sum_{h \in \Gamma} x_{h} a^{k} h\right) \widehat{G}_{i}, x_{h} \in R$.

Analisemos assim uma palavra de $(R G) a^{k} e_{i}$. Se apenas um coeficiente $x_{h} a^{k}$ desta palavra é diferente de 0 , para algum $h \in \Gamma$, teríamos $x_{h} a^{k} h \widehat{G}_{i} \in(R G) e_{i}$. Assim, existe $\beta \in R G$ tal que $x_{h} a^{k} h \widehat{G}_{i}=\beta a^{k} e_{i}$. Logo, $x_{h} a^{k} h \widehat{G}_{i} \cdot \widehat{G}_{i-1}=\beta a^{k} e_{i} \cdot \widehat{G}_{i-1}$. Como $e_{i} \cdot \widehat{G}_{i-1}=0$, temos que $x_{h} a^{k} h \widehat{G}_{i-1}=0$ e, assim, $x_{h} a^{k}=0$. Logo, $x_{h} a^{k} h \widehat{G}_{i}=0$, o que é uma contradição. Portanto, $w\left((R G) a^{k} e_{i}\right) \geq 2\left|G_{i}\right|$.

Agora, considere $\alpha \in G_{i-1} \backslash G_{i}$. Temos $(1-\alpha) a^{k} e_{i} \in(R G) a^{k} e_{i}$. Como

$$
\begin{aligned}
(1-\alpha) a^{k} e_{i} & =(1-\alpha) a^{k}\left(\widehat{G}_{i}-\widehat{G}_{i-1}\right) \\
& =(1-\alpha) a^{k} \widehat{G}_{i}-(1-\alpha) a^{k} \widehat{G}_{i-1} \\
& =(1-\alpha) a^{k} \widehat{G}_{i}
\end{aligned}
$$

e $\operatorname{supp}\left(a^{k} \widehat{G}_{i}\right) \cap \operatorname{supp}\left(a^{k} \alpha \widehat{G}_{i}\right)=\emptyset$, temos que $w\left((R G) a^{k} e_{i}\right) \leq 2\left|G_{i}\right|$. Portanto, $w\left((R G) a^{k} e_{i}\right)=2\left|G_{i}\right|$, para $i \neq 0$.

Consideremos agora o caso em que $i=0$. Temos

$$
\begin{aligned}
R G a^{k} e_{0} & =\left(R a^{k}\right)(G \widehat{G}) \\
& =\left(R a^{k}\right) \widehat{G} \\
& =\left\{r a^{k}\left(1+g_{0}+\ldots+g_{0}^{p^{n}-1}\right) \mid r \in R\right\}
\end{aligned}
$$

Assim, todos os elementos não nulos deste conjunto têm peso $p^{n}=|G|$. 
O próximo teorema é um caso particular do resultado a seguir. Foi incluído apenas porque, nesta situação mais simples, é fácil ver a origem das ideias envolvidas.

Teorema 3.0.16 Considere $R$ e $G$ nas condições da Hipótese A. Sejam $I_{r}=(R G) a^{k_{r}} e_{r}$, com $0 \leq k_{r} \leq t-1$. Considere $I_{i} \oplus I_{j}$, com $i<j$. Então $w\left(I_{i} \oplus I_{j}\right)=2\left|G_{j}\right|$ ou $\left|G_{1}\right|$.

Prova: Consideremos inicialmente o caso em que $i \neq 0$. Temos $G_{j} \subset G_{i}$. Seja $\alpha \in\left(I_{i} \oplus I_{j}\right)$. Assim, $\alpha=\alpha_{i} a^{k_{1}} e_{i}+\alpha_{j} a^{k_{2}} e_{j}$, onde $\alpha_{i}, \alpha_{j} \in R G$. Portanto,

$$
\alpha . \widehat{G}_{j}=\alpha_{i} a^{k_{1}}\left(\widehat{G}_{i}-\widehat{G}_{i-1}\right) \widehat{G}_{j}+\alpha_{j} a^{k_{2}}\left(\widehat{G}_{j}-\widehat{G}_{j-1}\right) \widehat{G}_{j}=\alpha
$$

Logo, $\alpha \in(R G) \widehat{G}_{j}$. Considere agora $\Gamma_{j}$ um transversal de $G_{j}$ em $G$. Assim, $\alpha=\left(\sum_{h \in \Gamma} x_{h} h\right) \widehat{G}_{j}$, onde $x_{h} \in R$. Suponhamos que apenas um coeficiente $x_{h} \neq 0$. Assim, $\alpha=x_{h} h \widehat{G}_{j}$. Como $e_{i} \cdot \widehat{G}_{i-1}=e_{j} \cdot \widehat{G}_{i-1}=0$, temos que $\alpha \cdot \widehat{G}_{i_{1}}=x_{h} h \widehat{G}_{j} \cdot \widehat{G}_{i-1}$. Logo, $x_{h} h \widehat{G}_{i-1}=0$ e, com isso, temos $x_{h}=0$, o que é uma contradição. Portanto, $w\left(I_{i} \oplus I_{j}\right) \geq 2\left|G_{j}\right|$. Como $w(I) \leq$ $w\left(I_{j}\right)=2\left|G_{j}\right|$, temos que $w(I)=2\left|G_{j}\right|$.

Provemos agora, o caso em $i=0$ e $1<j$. Como $I_{j} \subset I_{0}+I_{j}$, temos que $2\left|G_{j}\right|=w\left(I_{j}\right) \geq w\left(I_{0}+I_{j}\right)$.

Se $\alpha \in I_{0}+I_{j}$, então $\alpha=\alpha_{1} a^{k_{1}} \widehat{G}+\alpha_{2} a^{k_{2}}\left(\widehat{G}_{j}-\widehat{G}_{j-1}\right)$, onde $\alpha_{1}, \alpha_{2} \in R G$. Assim, $\alpha . \widehat{G}_{j}=\alpha$ e, portanto, $I_{0}+I_{j} \subset(R G) \widehat{G}_{j}$.

Seja $\Gamma$ um transversal de $G_{j}$ em $G$. Assim, podemos escrever $\alpha$ como sendo $\alpha=\left(\sum_{h \in \Gamma} x_{h} h\right) \widehat{G}_{j}$. Suponhamos que apenas um coeficiente $x_{h}$ seja diferente de zero. Assim, $\alpha=x_{h} h \widehat{G}_{j}=\alpha_{1} a^{k_{1}} \widehat{G}+\alpha_{2} a^{k_{2}}\left(\widehat{G}_{j}-\widehat{G}_{j-1}\right)$.

Como $G_{j} \subset \widehat{G}_{j-1}$, temos que $\widehat{G}_{j} \cdot \widehat{G}_{j-1}=\widehat{G}_{j-1}$. Assim, $x_{h} h \widehat{G}_{j} \cdot \widehat{G}_{j-1}=\alpha_{1} a^{k_{1}} \widehat{G} \widehat{G}_{j-1}+$ $\alpha_{2} a^{k_{2}}\left(\widehat{G}_{j}-\widehat{G}_{j-1}\right) \widehat{G}_{j-1}$, ou seja, $x_{h} h \widehat{G}_{j-1}=\alpha_{1} a^{k_{1}} \widehat{G}$. Como $\alpha_{1}=\sum r_{g} g$, podemos reescrever a última igualdade como $x_{h} h \widehat{G}_{j-1}=\sum r_{g} a^{k_{1}} \widehat{G}$. Desenvolvendo a equação anterior temos

$$
\sum_{g \in\left\{h G_{j-1}\right\}}\left(x_{h}-\sum r_{g} a^{k_{1}}\right) g-\sum_{g \in\left\{G / h G_{j-1}\right\}}\left(\sum r_{g} a^{k_{1}}\right) g=0
$$


Assim, $\sum r_{g} a^{k_{1}}=0$ e daí $x_{h}=0$, o que contradiz nossa hipótese inicial sobre $x_{h}$.

Concluímos assim que, para toda palavra $\alpha \in I_{0}+I_{j}$, com $\alpha=\left(\sum_{h \in \Gamma} x_{h} h\right) \widehat{G}_{j}$, devem existir pelo menos dois coeficientes $x_{h_{0}}, x_{h_{1}}$, com $h_{0}, h_{1} \in \Gamma$ diferentes de zero. Portanto, $w\left(I_{0}+I_{j}\right) \geq 2\left|G_{j}\right|$, e assim, $w\left(I_{0}+I_{j}\right)=2\left|G_{j}\right|$.

Provemos agora o caso em que $i=0$ e $j=1$.

Seja $\alpha \in C$. Como $C=I_{0} \oplus I_{1}$, existem $\beta_{0}, \beta_{1} \in R G$, tais que $\alpha=\beta_{0} a^{k_{0}} e_{0}+\beta_{1} a^{k_{1}} e_{1}$. Como $\alpha \cdot \widehat{G}_{1}=\alpha$, temos $I \subset R G \widehat{G}_{1}$ e, assim, $w(I) \geq w\left(R G \widehat{G}_{1}\right)=\left|G_{1}\right|$.

Seja $k=\max \left\{k_{0}, k_{1}\right\}$. Temos

$$
R G a^{k} \widehat{G}_{1}=R G a^{k} \widehat{G} \oplus R G a^{k}\left(\widehat{G}_{1}-\widehat{G}\right) \subset R G a^{k_{0}} e_{0} \oplus R G a^{k_{1}} e_{1}=I
$$

$\operatorname{Logo}\left|G_{1}\right|=w\left(R G a^{k} \widehat{G}_{1}\right) \geq w(I)$. Portanto, $w(I)=\left|G_{1}\right|$.

Teorema 3.0.17 Considere $R$ e $G$ nas condições da Hipótese A. Sejam $I_{j}=(R G) a^{k_{j}} e_{j}$, $\operatorname{com} 0 \leq k_{j} \leq t-1$ e $I=I_{0} \oplus \ldots \oplus I_{j}$, com $0 \leq j \leq n-1$. Então $w\left(I_{0} \oplus I_{1} \oplus \ldots \oplus I_{j}\right)=\left|G_{j}\right|$.

Prova: Seja $\alpha \in I$. Como $I=I_{0} \oplus \ldots \oplus I_{j}$, existem $\beta_{0}, \ldots, \beta_{j} \in R G$ tais que $\alpha=\beta_{0} a^{k_{0}} \widehat{G}+$ $\ldots+\beta_{j} a^{k_{j}}\left(\widehat{G}_{j}-\widehat{G}_{j-1}\right)$. Como $G_{j} \subset G_{i}$, para $1 \leq i \leq j-1$, temos que $\widehat{G}_{j} \cdot e_{i}=e_{i}$, para $0 \leq i \leq j-1$ e assim, $\alpha \cdot \widehat{G}_{j}=\alpha$. Portanto, $I \subset(R G) \widehat{G}_{j}$ e $w(I) \geq w\left((R G) \widehat{G}_{j}\right)=\left|G_{j}\right|$.

Seja $k=\max \left\{k_{0}, \ldots, k_{j}\right\}$. Logo,

$$
(R G) a^{k} \widehat{G}_{j} \subset(R G) a^{k} \widehat{G} \oplus(R G) a^{k}\left(\widehat{G}_{1}-\widehat{G}\right) \oplus \ldots \oplus(R G) a^{k}\left(\widehat{G}_{j}-\widehat{G}_{j-1}\right) \subset I_{0} \oplus I_{1} \oplus \ldots \oplus I_{j}
$$

Portanto, $\left|G_{j}\right|=w\left((R G) a^{k} \widehat{G}_{j}\right) \geq w(I)$. Logo, $w(I)=\left|G_{j}\right|$.

Teorema 3.0.18 Considere $R$ e $G$ nas condições da Hipótese A. Sejam $I_{j}=(R G) a^{k_{j}} e_{j}$, com $0 \leq k_{j} \leq t-1$. Se $I=I_{j_{1}} \oplus \ldots \oplus I_{j_{l}}, j_{r}<j_{r+1}$, para $1 \leq r \leq l \operatorname{com}\left\{j_{1}, \ldots, j_{l}\right\} \varsubsetneqq$ $\left\{0,1, \ldots, j_{l}\right\}$, então $w(I)=2\left|G_{j_{l}}\right|$. 
Prova: Consideremos inicialmente

$$
I=I_{j_{1}} \oplus \ldots \oplus I_{j_{l}}, \text { com } j_{1} \neq 0 \text { e } j_{r}<j_{r+1} \text {, para } 1 \leq r \leq l
$$

Seja $\alpha \in I . \quad$ Como $I=I_{j_{1}} \oplus \ldots \oplus I_{j_{l}}$, existem $\beta_{j_{1}}, \ldots, \quad \beta_{j_{l}}$, tais que

$$
\alpha=\beta_{j_{1}} a^{k_{j_{1}}}\left(\widehat{G}_{j_{1}}-\widehat{G}_{j_{1}-1}\right)+\ldots+\beta_{j_{l}} a^{k_{j_{l}}}\left(\widehat{G}_{j_{l}}-\widehat{G}_{j_{l-1}}\right) .
$$

Como $G_{j_{l}} \subset G_{j_{i}}$, para $1 \leq i \leq l-1$, temos $\widehat{G}_{j_{l}} \cdot e_{j_{i}}=e_{j_{i}}$, para $1 \leq i \leq l$. Assim, $\alpha G_{j_{l}}=\alpha$ e, portanto, $I \subset(R G) \widehat{G}_{j_{l}}$. Seja $\Gamma$ um transversal de $G_{j_{l}}$ em $G$. Assim, podemos escrever $\alpha=\left(\sum_{h \in \Gamma} x_{h} h\right) \widehat{G}_{j_{l}}, x_{h} \in R$. Suponhamos agora que apenas um $x_{h_{0}}$ seja diferente de zero, para $\operatorname{algum} h_{0} \in \Gamma$. Assim,

$$
\alpha=x_{h_{0}} h_{0} \widehat{G}_{j_{l}}=\beta_{j_{1}} a^{k_{j_{1}}}\left(\widehat{G}_{j_{1}}-\widehat{G}_{j_{1}-1}\right)+\ldots+\beta_{j_{l}} a^{k_{j_{l}}}\left(\widehat{G}_{j_{l}}-\widehat{G}_{j_{l-1}}\right)
$$

Como $\widehat{G}_{j_{i}} \subset \widehat{G}_{j_{1}-1}$, para $1 \leq i \leq l$, temos que $\widehat{G}_{j_{i}} \cdot \widehat{G}_{j_{1}-1}=\widehat{G}_{j_{1}-1}$, para $1 \leq i \leq l$. Logo, $\widehat{G}_{j_{1}-1} \cdot e_{j_{i}}=0$, para $1 \leq i \leq l$. Multiplicando ambos os lados da igualdade por $\widehat{G}_{j_{1}-1}$, temos $x_{h_{0}} h_{0} \widehat{G}_{j_{1}-1}=0$. A partir daí, temos $x_{h_{0}}=0$, o que contradiz nossa escolha inicial de $x_{h_{0}} \neq 0$. Portanto, $w(I) \geq 2\left|G_{j_{l}}\right|$.

Como $I_{j_{l}} \subset I$, temos $2\left|G_{j_{l}}\right|=w\left(I_{j_{l}}\right) \geq w(I)$. Logo, $w(I)=2\left|G_{j_{l}}\right|$.

Agora considere

$$
I=I_{0} \oplus I_{j_{1}} \oplus \ldots \oplus I_{j_{l}}, \text { com } j_{r}<j_{r+1} \text { para } 1 \leq r \leq l \text { e }\left\{j_{1}, \ldots, j_{l}\right\} \varsubsetneqq\left\{1, \ldots, j_{l}\right\}
$$

Como $I_{j_{l}} \subset I$, temos que $2\left|G_{j_{l}}\right|=w\left(I_{j_{l}}\right) \geq w(I)$.

Seja $\alpha \in I$. Como $I=I_{0} \oplus I_{j_{1}} \oplus \ldots \oplus I_{j_{l}}$, existem $\beta_{0}, \beta_{j_{1}}, \ldots, \beta_{j_{l}} \in R G$ tais que $\alpha=\beta_{0} a^{k_{0}} \widehat{G}+\beta_{j_{1}} a^{k_{j_{1}}}\left(\widehat{G}_{j_{1}}-\widehat{G}_{j_{1}-1}\right)+\ldots+\beta_{j_{l}} a^{k_{j_{l}}}\left(\widehat{G}_{j_{l}}-\widehat{G}_{j_{l-1}}\right)$. Assim, $\alpha \cdot \widehat{G}_{j_{l}}=\alpha$. Logo, $I \subset(R G) \widehat{G}_{j_{l}}$. 
Seja $\Gamma$ um transversal de $G_{j_{l}}$ em $G$. Assim, podemos escrever $\alpha=\left(\sum_{h \in \Gamma} x_{h} h\right) \widehat{G}_{j_{l}}$, onde $x_{h} \in R$. Suponha que exista apenas um $h_{0} \in \Gamma$ tal que $x_{h_{0}} \neq 0$. Assim,

$$
\alpha=x_{h_{0}} t_{0} \widehat{G}_{j_{l}}=\beta_{0} a^{k_{0}} \widehat{G}+\beta_{j_{1}} a^{k_{j_{1}}}\left(\widehat{G}_{j_{1}}-\widehat{G}_{j_{1}-1}\right)+\ldots+\beta_{j_{l}} a^{k_{j_{l}}}\left(\widehat{G}_{j_{l}}-\widehat{G}_{j_{l-1}}\right) .
$$

Como $\left\{j_{1}, \ldots, j_{l}\right\} \varsubsetneqq\left\{1, \ldots, j_{l}\right\}$, existe $r \in\left\{1, \ldots, j_{l}\right\}$, tal que $r \notin\left\{j_{1}, \ldots, j_{l}\right\}$. Tome $r$ como sendo o menor índice pertencente à $\left\{1, \ldots, j_{l}\right\}$ e que não pertence à $\left\{j_{1}, \ldots, j_{l}\right\}$.

Multiplicando ambos os lados da igualdade acima por $\widehat{G}_{r}$, temos

$$
\left.x_{h_{0}} h_{0} \widehat{G}_{r}=\beta_{0} a^{k_{0}} \widehat{G}+\beta_{1} a^{k_{1}}\left(e_{1}\right)+\ldots+\beta_{r-1} a^{k_{r-1}}\left(e_{r-1}\right)\right)
$$

Portanto, $\alpha \widehat{G}_{r}$ pertence ao código $C^{\prime}$ gerado por

$$
<a^{k_{0}} \widehat{G}>\oplus<a^{k_{1}}\left(e_{1}\right)>\oplus \ldots \oplus<a^{k_{r-1}}\left(e_{r-1}\right)>
$$

Mas o peso de $\alpha \widehat{G}_{r}$ é dado por $w\left(\alpha \widehat{G}_{r}\right)=\left|G_{r}\right|$ e peso de $C^{\prime}$ é $w\left(C^{\prime}\right)=\left|G_{r-1}\right|$. Como $G_{r} \subset G_{r-1}$, temos que $\left|G_{r}\right|<\left|G_{r-1}\right|$, o que é uma contradição. Logo, $w(C) \geq 2\left|G_{j_{l}}\right| \mathrm{e}$ $\operatorname{assim} w(C)=2\left|G_{j_{l}}\right|$.

\subsection{Códigos que são livres como $R$-submódulos de $R G$}

Agora iremos caracterizar todos os códigos cíclicos de comprimento $p^{n}$ que são $R$ submódulos livres de $R G$. Para isso, lembremos os seguintes resultados.

Teorema 3.1.1 ( [18], Teorema 3.10) Um módulo $M$ é projetivo se, somente se, $M$ é um somando direto de um módulo livre.

Teorema 3.1.2 ( [18], Teorema 7.5) Se $R$ é um anel local, então todo módulo projetivo finitamente gerado sobre $R$ é livre. 
Como estamos trabalhando com módulos finitos, eles são finitamente gerados e assim qualquer módulo que for um somando de um módulo livre é projetivo e, portanto, livre.

O próximo resultado é semelhante a Proposição 2.1 provado por Dutra, Ferraz e Polcino Milies em [13] para algebras de grupo sobre corpos. Exibiremos uma base para $R G e_{i}$, provando assim que $R G e_{i}$ é um código livre. Mais adiante exibiremos outra base para $R G e_{i}$.

Teorema 3.1.3 Considere $R$ e $G$ nas condições da Hipótese A. Seja $\gamma$ um transversal de $G_{i-1}$ em $G$ e $\tau$ um transversal de $G_{i}$ em $G_{i-1}$. Então

$$
\mathcal{B}=\left\{c(1-h) \widehat{G}_{i} \mid c \in \gamma, h \in \tau \backslash\{1\}\right\}
$$

é uma base de $R G e_{i}$ sobre $R$.

Prova: Primeiramente provaremos que os elementos de $\mathcal{B}$ pertencem a $R G e_{i}$. Para isso, observe que:

1. Para $h \in \tau \backslash\{1\}$, temos $(1-h) \widehat{G}_{i-1}=0$, pois $h \widehat{G}_{i-1}=\widehat{G}_{i-1}$.

2. Para $c \in \gamma$ e $h \in \tau \backslash\{1\}$, temos $c(1-h) \widehat{G}_{i}=c(1-h) \widehat{G}_{i}\left(\widehat{G}_{i}-\widehat{G}_{i-1}\right)=c(1-h) \widehat{G}_{i} e_{i} \in$ $R G e_{i}$.

Mostremos agora que os elementos de $\mathcal{B}$ são linearmente independentes. Sejam $x_{c h} \in R$ tais que $0=\sum_{c, h} x_{c h}\left(c(1-h) \widehat{G}_{i}\right)$.

$$
0=\sum_{c, h} x_{c h}\left(c(1-h) \widehat{G}_{i}\right)=\sum_{c}\left(\sum_{h} x_{c h}\right) c \widehat{G}_{i}-\sum_{c, h} x_{c h} c h \widehat{G}_{i} .
$$

Agora observe que, para $c, h$ fixados, temos que o elemento $c h \widehat{G}_{i}$ tem suporte disjunto de qualquer outro elemento nesta combinação linear. De fato, como $\tau$ é um transversal de $G_{i}$ em $G_{i-1}$ e $h \in \tau \backslash\{1\}$, temos que $\widehat{G}_{i}$ e $h \widehat{G}_{i}$ têm suportes disjuntos. Como $\gamma$ é um transversal de $G_{i-1}$ em $G c \in \gamma$, temos que $c \widehat{G}_{i}$ e $c h \widehat{G}_{i}$ também têm suportes disjuntos. É 
claro que se $c_{j} \neq c_{k} \in \gamma$, então $c_{j} \widehat{G}_{i}$ e $c_{k} \widehat{G}_{i}$ têm suportes disjuntos. Como $\{c h, c \in \gamma$ e $h \in \tau\}$ formam um transversal de $G_{i}$ em $G$, temos que $c_{j} h_{j} \widehat{G}_{i}$ e $c_{k} h_{k} \widehat{G}_{i}$ têm suporte disjuntos, para $c_{j} \neq c_{k}$ ou $h_{j} \neq h_{k}$. Portanto, $x_{c h}=0$, para todo $c \in \gamma$ e $h \in \tau$. Devemos provar que o módulo livre sobre $R$ gerado por $\mathcal{B}$ é igual ao ideal gerado por $e_{i}$. Já provamos que todo elemento de $\mathcal{B}$ pertence a $R G e_{i}$. Provemos agora que os dois conjuntos têm o mesmo número de elementos. Pelo Teorema 3.0.14, $\left|R G e_{i}\right|=|\bar{R}|^{t\left(p^{i}-p^{i-1}\right)}=|R|^{\left(p^{i}-p^{i-1}\right)}$. Por outro lado, o número de elementos gerado pelo módulo livre sobre $R$ cuja base é $\mathcal{B}$ é dado por $|R|^{|\gamma|(|\tau|-1)}=|R|^{\left|\frac{G}{G_{i-1}}\right|\left(\left|\frac{G_{i-1}}{G_{i}}\right|-1\right)}=|R|^{p^{i}-p^{i-1}}$. Portanto, $R G e_{i}$ é livre e $\mathcal{B}$ é uma base de $R G e_{i}$

Corolário 3.1.4 O posto do código livre $R G e_{i}$ é $p^{i}-p^{i-1}$.

Observe que o ideal gerado por $a^{k} e_{i}$ não pode ser livre, pois $\left(a^{t-k} \alpha\right) \cdot\left(a^{k} e_{i}\right)=0$, para qualquer $\alpha \in R G e_{i}$.

Pelos Teoremas 3.1.1, 3.1.2 e 3.1.3, temos o seguinte corolário.

Corolário 3.1.5 Seja $G$ um grupo cíclico de ordem $p^{n}$ e $R$ um anel de cadeia com $|R|=q^{k}$, onde $q \nmid p^{n}$. Seja $e_{i}=\widehat{G}_{i}-\widehat{G}_{i-1}$. Então os possíveis códigos cíclicos livres de comprimento $p^{n}$ são da forma

$$
C=R G e_{i_{1}} \oplus \ldots \oplus R G e_{i_{k}}
$$

Corolário 3.1.6 Seja $G$ um grupo cíclico de ordem $p^{n}$ e $R$ um anel de cadeia com $|R|=q^{k}$, onde $q \nmid p^{n}$. O números de códigos cíclicos livres de comprimento $p^{n}$ sobre um anel de cadeia $R$ é $2^{n+1}$.

Pelo Corolário 3.1.4, posto $\left(R G e_{i}\right)=p^{i}-p^{i-1}$. Adiante exibiremos outra base para $R G e_{i}$, para a qual será necessária a seguinte proposição.

Proposição 3.1 .7 ( [18], Proposição 7.18 ) Seja $M$ um módulo livre sobre um anel comutativo de posto n. Então qualquer conjunto gerador de $n$ elementos é uma base de $M$. 
Teorema 3.1.8 Seja $G=<g \mid g^{p^{n}}=1>$. O conjunto $\mathcal{B}=\left\{e_{i}, g e_{i}, g^{2} e_{i}, \ldots ., g^{p^{i}-p^{i-1}-1} e_{i}\right\}$ é uma base de $R G e_{i}$.

Prova: Como o número de elementos de $\mathcal{B}$ é $p^{i}-p^{i-1}$, pela Proposição 3.1.7 basta provar que $\mathcal{B}$ gera $R G e_{i}$. Como um elemento de $R G e_{i}$ é dado por $r_{0} e_{i}+r_{1} g e_{i}+\ldots+r_{p^{n}-1} g^{p^{n}-1} e_{i}$, devemos provar então que os elementos da forma $r_{l} g^{l} e_{i}$, onde $p^{i}-p^{i-1} \leq l \leq p^{n-1}$, são combinações lineares de elementos de $\mathcal{B}$. Observe porém que $g^{p^{i}} \cdot e_{i}=e_{i}$, pois

$$
g^{p^{i}} e_{i}=g^{p^{i}} \widehat{G}_{i}-g^{p^{i}} \widehat{G}_{i-1}=\widehat{G}_{i}-\widehat{G}_{i-1} .
$$

Logo, $g^{p^{i}+k} e_{i}=g^{k} e_{i}, 0 \leq k \leq p^{n}-p^{i}+p^{i+1}-1$, donde um elemento $\alpha \in R G e_{i}$ é da forma $\alpha=\sum_{j=0}^{p^{i}} r g_{0}^{j} e_{i}$. Portanto, é suficiente provar que elementos da forma $g^{j} e_{i}$ com $p^{i}-p^{i-1} \leq j \leq p^{i}$ são combinações lineares dos elementos de $\mathcal{B}$.

Escrevendo de outra maneira, é suficiente provar que os elementos da forma $g^{(p-1) p^{i-1}+k} e_{i}$ onde $0 \leq k<p^{i-1}$ são combinações lineares dos elementos de $\mathcal{B}$.

Note que

$$
\begin{aligned}
e_{i}= & \frac{1}{p^{n-i+1}}\left((p-1)-g^{p^{i-1}}-g^{2 p^{i-1}}-\ldots+(p-1) g^{p \cdot p^{i-1}}-g^{(p+1) p^{i-1}}-g^{(p+2) p^{i-1}}-\ldots\right. \\
& \left.\ldots+(p-1) g^{2 p \cdot p^{i-1}}-\ldots-g^{\left(p^{n-i+1}-(p-1)\right) p^{i-1}}-g^{\left(p^{n-i+1}-1\right) p^{i-1}}\right) .
\end{aligned}
$$

Logo,

$$
\begin{aligned}
g^{(p-1) p^{i-1}+k} e_{i}= & \frac{1}{p^{n-i+1}}\left((p-1) g^{(p-1) p^{i-1}+k}-g^{p \cdot p^{i-1}+k}-g^{(p+1) p^{i-1}+k}-\ldots-g^{(2 p-2) \cdot p^{i-1}+k}+\right. \\
& +(p-1) g^{(2 p-1) p^{i-1}+k}-g^{(2 p) p^{i-1}+k}-g^{(2 p+1) p^{i-1}+k}-\ldots+ \\
& \left.+(p-1) g^{\left(p^{n-i+1}\right) \cdot p^{i-1+k}}-g^{k}-g^{p^{i-1}+k}-g^{2 p^{i-1}+k}+\ldots-g^{(p-2) p^{i-1}+k}\right) .
\end{aligned}
$$

Para cada índice $k$ escrito acima, temos que $g^{(p-1) p^{i-1}+k} e_{i}$ é combinação linear do conjunto $\mathcal{B}^{\prime}=\left\{g^{k} e_{i}, g^{p^{i-1}+k} e_{i}, \ldots, g^{(p-2) p^{i-1}+k} e_{i}\right\}$. Para isso, escrevemos:

$$
\begin{aligned}
g^{k} e_{i}= & \frac{1}{p^{n-i+1}}\left((p-1) g^{k}-g^{p^{i-1}+k}-g^{2 p^{i-1}+k}-\ldots-g^{(p-1) p^{i-1}+k}+(p-1) g^{p \cdot p^{i-1}+k}-\right. \\
& -g^{(p+1) p^{i-1}+k}-\ldots+(p-1) g^{(2 p) \cdot p^{i-1}+k}-\ldots-(p-1) g^{\left(p^{n-i+1}-p\right) p^{i-1}+k}- \\
& \left.-g^{\left(p^{n-i+1}-(p-1)\right) p^{i-1}+k}-g^{\left(p^{n-i+1}-(p-2)\right) p^{i-1}+k}-\ldots-g^{\left(p^{n-i+1}-1\right) p^{i-1}+k}\right) .
\end{aligned}
$$




$$
\begin{aligned}
& g^{\left(p^{i-1}+k\right)} e_{i}=\frac{1}{p^{n-i+1}}\left((p-1) g^{p^{i-1}+k}-g^{2 p^{i-1}+k}-g^{3 p^{i-1}+k}-\ldots-g^{p \cdot p^{i-1}+k}+\right. \\
& +(p-1) g^{(p+1) p^{i-1}+k}-g^{(p+2) p^{i-1}+k}-g^{(p+3) p^{i-1}+k}-\ldots+(p-1) g^{(2 p+1) p^{i-1}+k}- \\
& \left.\ldots+(p-1) g^{\left(p^{n-i+1}-(p-1)\right) p^{i-1}+k}-g^{\left(p^{n-i+1}-(p-2)\right) p^{i-1}+k}-\ldots .-g^{k}\right) . \\
& g^{\left(2 p^{i-1}+k\right)} e_{i}=\frac{1}{p^{n-i+1}}\left((p-1) g^{2 p^{i-1}+k}-g^{3 p^{i-1}+k}-g^{4 p^{i-1}+k}-\ldots-g^{(p+1) \cdot p^{i-1}+k}+\right. \\
& +(p-1) g^{(p+2) p^{i-1}+k}-g^{(p+3) p^{i-1}+k}-g^{(p+4) p^{i-1}+k}-\ldots+(p-1) g^{(2 p+2) p^{i-1}+k}- \\
& \left.\ldots+(p-1) g^{\left(p^{n-i+1}-(p-2)\right) p^{i-1}+k}-g^{\left(p^{n-i+1}-(p-3)\right) p^{i-1}+k}-\ldots . g^{k}-g^{p^{i-1}+k}\right) \\
& g^{(p-2) p^{i-1}+k} e_{i}=\frac{1}{p^{n-i+1}}\left((p-1) g^{(p-2) p^{i-1}+k}-g^{(p-1) p^{i-1}+k}-\ldots-g^{(2 p-3) p^{i-1}+k}\right. \\
& +(p-1) g^{(2 p-2) p^{i-1}+k}-g^{(2 p-1) p^{i-1}+k}-\ldots+(p-1) g^{(3 p-2) p^{i-1}+k}-\ldots \\
& +(p-1) g^{\left(p^{n-i+1}-2\right) p^{i-1}+k}-g^{\left(p^{n-i+1}-1\right) p^{i-1}+k}-g^{k}-g^{p^{i-1}+k}-\ldots \\
& \left.-g^{(p-3) p^{i-1}+k}\right) \text {. }
\end{aligned}
$$

Agora, pode se verificar diretamente a igualdade

$$
g^{(p-1) p^{i-1}+k} e_{i}=-\left(g^{k} e_{i}\right)-\left(g^{p^{i-1}+k} e_{i}\right)-\left(g^{2 p^{i-1}+k} e_{i}\right)-\ldots-\left(g^{(p-2) p^{i-1}+k} e_{i}\right) .
$$

Como $\mathcal{B}^{\prime} \subset \mathcal{B}$, segue que $\left\{e_{i}, g e_{i}, \ldots g^{(p-1) p^{i-1}-1} e_{i}\right\}$ é um conjunto gerador de $R G e_{i}$.

Exemplo 3.1.9 Seja $G$ um grupo cíclico de ordem 25. Neste caso,

$$
e_{2}=\frac{1}{5}\left(4-g^{5}-g^{10}-g^{15}-g^{20}\right)
$$

Considere a seguinte tabela com todos elementos da forma $g^{k} e_{2}$, com $0 \leq k \leq 24$. 


\begin{tabular}{|l|l|}
\hline$e_{2}=\frac{1}{5}\left(4-g^{5}-g^{10}-g^{15}-g^{20}\right)$ & $g^{13} e_{2}=\frac{1}{5}\left(4 g^{13}-g^{18}-g^{23}-g^{3}-g^{8}\right)$ \\
$g e_{2}=\frac{1}{5}\left(4 g-g^{6}-g^{11}-g^{16}-g^{21}\right)$ & $g^{14} e_{2}=\frac{1}{5}\left(4 g^{14}-g^{19}-g^{24}-g^{4}-g^{9}\right)$ \\
$g^{2} e_{2}=\frac{1}{5}\left(4 g^{2}-g^{7}-g^{12}-g^{17}-g^{22}\right)$ & $g^{15} e_{2}=\frac{1}{5}\left(4 g^{15}-g^{20}-1-g^{5}-g^{10}\right)$ \\
$g^{3} e_{2}=\frac{1}{5}\left(4 g^{3}-g^{8}-g^{13}-g^{18}-g^{23}\right)$ & $g^{16} e_{2}=\frac{1}{5}\left(4 g^{16}-g^{21}-g-g^{6}-g^{11}\right)$ \\
$g^{4} e_{2}=\frac{1}{5}\left(4 g^{4}-g^{9}-g^{14}-g^{19}-g^{24}\right)$ & $g^{17} e_{2}=\frac{1}{5}\left(4 g^{17}-g^{22}-g^{2}-g^{7}-g^{12}\right)$ \\
$g^{5} e_{2}=\frac{1}{5}\left(4 g^{5}-g^{10}-g^{15}-g^{20}-1\right)$ & $g^{18} e_{2}=\frac{1}{5}\left(4 g^{18}-g^{23}-g^{3}-g^{8}-g^{13}\right)$ \\
$g^{6} e_{2}=\frac{1}{5}\left(4 g^{6}-g^{11}-g^{16}-g^{21}-g\right)$ & $g^{19} e_{2}=\frac{1}{5}\left(4 g^{19}-g^{24}-g^{4}-g^{9}-g^{14}\right)$ \\
$g^{7} e_{2}=\frac{1}{5}\left(4 g^{7}-g^{12}-g^{17}-g^{22}-g^{2}\right)$ & $g^{20} e_{2}=\frac{1}{5}\left(4 g^{20}-1-g^{5}-g^{10}-g^{15}\right)$ \\
$g^{8} e_{2}=\frac{1}{5}\left(4 g^{8}-g^{13}-g^{18}-g^{23}-g^{3}\right)$ & $g^{21} e_{2}=\frac{1}{5}\left(4 g^{21}-g-g^{6}-g^{11}-g^{16}\right)$ \\
$g^{9} e_{2}=\frac{1}{5}\left(4 g^{9}-g^{14}-g^{19}-g^{24}-g^{4}\right)$ & $g^{22} e_{2}=\frac{1}{5}\left(4 g^{22}-g^{2}-g^{7}-g^{12}-g^{17}\right)$ \\
$g^{10} e_{2}=\frac{1}{5}\left(4 g^{10}-g^{15}-g^{20}-1-g^{5}\right)$ & $g^{23} e_{2}=\frac{1}{5}\left(4 g^{23}-g^{3}-g^{8}-g^{13}-g^{18}\right)$ \\
$g^{11} e_{2}=\frac{1}{5}\left(4 g^{11}-g^{16}-g^{21}-g-g^{6}\right)$ & $g^{24} e_{2}=\frac{1}{5}\left(4 g^{24}-g^{4}-g^{9}-g^{14}-g^{19}\right)$ \\
$g^{12} e_{2}=\frac{1}{5}\left(4 g^{12}-g^{17}-g^{22}-g^{2}-g^{7}\right)$ &
\end{tabular}

Temos que $\mathcal{B}=\left\{e_{2}, g e^{2}, \ldots, g^{19} e_{2}\right\}$ é uma base para $<e_{2}>$. Note que os elementos da forma $g^{k} e_{2}$, com $20 \leq k \leq 24$ são dados pelas seguintes combinações dos elementos de $\mathcal{B}$.

$$
\begin{aligned}
& g^{20} e_{2}=-e_{2}-g^{5} e_{2}-g^{10} e_{2}-g^{15} e_{2} \\
& g^{21} e_{2}=-g e_{2}-g^{6} e_{2}-g^{11} e_{2}-g^{16} e_{2} \\
& g^{22} e_{2}=-g^{2} e_{3}-g^{7} e_{2}-g^{12} e_{2}-g^{17} e_{2} \\
& g^{23} e_{2}=-g^{3} e_{2}-g^{8} e_{2}-g^{13} e_{2}-g^{18} e_{2} \\
& g^{24} e_{2}=g^{4} e_{2}-g^{9} e_{2}-g^{14} e_{2}-g^{19} e_{2}
\end{aligned}
$$

\subsection{Códigos MDS de Comprimento $p^{n}$}

Já é conhecido que, para códigos $C$ de comprimento $n$ sobre qualquer alfabeto de tamanho $m$, vale a seguinte desigualdade

$$
d_{H}(C) \leq n-\log _{m}(|C|)+1
$$


Definição 3.2.1 Dizemos que um código $C$ de comprimento $n$ sobre um alfabeto de tamanho $m$ é um código Separável pela Distância Máxima, ou código MDS, se

$$
d_{H}(C)=n-\log _{m}(|C|)+1
$$

Até aqui já caracterizamos os códigos de comprimento $p^{n}$ sobre anéis de cadeia, seus pesos, tamanhos e alguns resultados sobre posto. Vejamos alguns exemplos de códigos e uma comparação com o limitante antes mencionado.

Exemplo 3.2.2 Considere o anel $R G$, onde $R=\mathbb{Z}_{2^{4}}$ e $G=C_{5^{3}}$. Neste anel os idempotentes são $e_{0}=\widehat{G}, e_{1}=\widehat{G}_{1}-\widehat{G}, e_{2}=\widehat{G}_{2}-\widehat{G}_{1}$ e $e_{3}=\widehat{G}_{3}-\widehat{G}_{2}$, onde $G_{i}=<g^{5^{i}}>$.

Seja $C=R G e_{1} \oplus R G e_{2}$. Temos que

$$
\begin{gathered}
w(C)=2\left|G_{2}\right|=2 \cdot 5^{3-2}=10 \\
|C|=2^{4\left(5^{1}-5^{0}\right)+4\left(5^{2}-5^{1}\right)}=2^{96} .
\end{gathered}
$$

Pela desigualdade 3.2, para o código $C=<e_{1}>\oplus<e_{2}>$, temos:

$$
d_{H}(C) \leq 125-\log _{2^{4}}\left(2^{96}\right)+1=102 .
$$

Neste exemplo o peso está muito distante do seu limitante superior

Exemplo 3.2.3 Considere agora $G=<g>$ um grupo cíclico de ordem 9 e o anel $R=\mathbb{Z}_{4}$. Observe que $U\left(\mathbb{Z}_{9}\right)=\{\overline{1}, \overline{2}, \overline{4}, \overline{5}, \overline{7}, \overline{8}\}$ e $<\overline{2}>=U\left(\mathbb{Z}_{9}\right)$. Como $\frac{\left|\mathbb{Z}_{4}\right|}{|<2>|}=2$, temos que $e_{0}, e_{1}$ e $e_{2}$ são idempotentes primitivos ortogonais, onde $e_{0}=\widehat{G}, e_{1}=\widehat{G}_{1}-\widehat{G}_{0}$ e $e_{2}=\widehat{G}_{2}-\widehat{G}_{1}$, onde $G_{i}=<g^{3^{i}}>$.

Tomemos $C=<e_{1}>$. Assim,

$$
w(C)=2\left|G_{1}\right|=2.3=6
$$


e

$$
|C|=2^{2(3-1)}=2^{4}
$$

O limitante para $C$ é dado por

$$
d_{H}(C) \leq 9-\log _{4}\left(2^{4}\right)+1=8
$$

Neste caso, a distância do código está proxima da distância máxima do limitante.

Tomemos agora $G$ um grupo cíclico de ordem $4, R$ um anel de cadeia tal que $|\bar{R}|=q$, onde $q \equiv 3(\bmod 4)$ e o código gerado por $C=<e_{1}>$, onde $e_{1}=\widehat{G}_{1}-\widehat{G}_{0}$. Temos que

$$
\begin{gathered}
W(C)=2\left|G_{1}\right|=2 \cdot 2=4, \\
|C|=|\bar{R}|^{t\left(2^{1}-2^{1-1}\right)}=q^{t} .
\end{gathered}
$$

e

$$
d_{H}(C) \leq 4-\log _{|R|}\left(|\bar{R}|^{t}\right)+1=4-\log _{q^{t}}\left(q^{t}\right)=4=w(C)
$$

Observe que, para qualquer $q \equiv 3(\bmod 4)$, estes códigos tem a maior distância de Hamming possível, além disso, é possível aumentar o número de palavras do código aumentando $q$. Sendo assim, qualquer outro código de comprimento 4, com $q^{t}$ elementos possui distância de Hamming menor ou igual a deste código. Além disso, como $<\bar{q}>=U\left(\mathbb{Z}_{4}\right)$, temos que $\left\{e_{0}, e_{1}, e_{2}\right\}$ é o conjunto dos idempotentes primitivos ortogonais em $R G$.

Exemplo 3.2.4 Sejam $G$ um grupo cíclico de ordem $4, R=\mathbb{Z}_{11^{7}}$ um anel de cadeia e o código $C_{1}$ gerado por $e_{1}=\widehat{G}_{1}-\widehat{G}_{0}$. Temos que $\frac{\left|\mathbb{Z}_{117}\right|}{|<\overline{11}>|}=11$. Como $\overline{11}=\overline{3}$ em $\mathbb{Z}_{4}$, temos 
$q u e<\overline{11}>=U\left(\mathbb{Z}_{4}\right)$. Logo,

$$
w\left(C_{1}\right)=2\left|G_{1}\right|=4
$$

e

$$
\left|C_{1}\right|=11^{7}
$$

O limitante da distância de Hamming é dado por

$$
d_{H}\left(C_{1}\right) \leq 4-\log _{11^{7}}\left(11^{7}\right)+1=4=w(C)
$$

Observe que $C_{2}=<e_{2}>$ não é um código MDS. De fato,

$$
w\left(R G e_{2}\right)=2.2^{2-2}=2 \text { e } d_{H}\left(R G e_{2}\right) \leq 4-2^{2}-2^{1}+1=3 .
$$

Observe que o código $C_{3}=R G e_{0} \oplus R G e_{1}$ também não é $M D S$. De fato, $w\left(C_{3}\right)=\left|G_{1}\right|=2^{2-1}=2$. O limitante de Hamming neste caso é dado por

$$
d_{H}\left(C_{3}\right) \leq 4-\log _{|R|}(|\bar{R}|)^{(t(2-1)+t)}+1=5-2=3 .
$$

Agora, porém, o código $C_{4}=R G e_{0} \oplus R G e_{2}$ é um código MDS. De fato, $w\left(C_{4}\right)=2 \cdot\left|G_{2}\right|=2 \cdot 2^{(2-2)}=2$. O limitante de Hamming é dado por

$$
d_{H}\left(C_{4}\right)=5-\log _{|R|}(|\bar{R}|)^{t\left(2^{2}-2^{1}\right)+t}=5-3=2 .
$$

Novamente, o código $C_{5}=R G e_{1} \oplus R G e_{2}$ é um código MDS. De fato, $w\left(C_{5}\right)=2 .\left|G_{2}\right|=2.2^{(2-2)}=2$. O limitante de Hamming é dado por

$$
d_{H}\left(C_{5}\right) \leq 5-\log _{|R|}(|\bar{R}|)^{t\left(2^{1}-2^{0}\right)+t\left(2^{2}-2^{1}\right)}=5-3=2
$$




\subsection{Códigos MDS de Comprimento $2^{n}$}

Análogo ao que foi feito anteriormente, iremos analisar códigos de comprimento $2^{n}$.

Quando tomamos $|\bar{R}|=q$, onde $\bar{q}$ gera $U\left(\mathbb{Z}_{p^{n}}\right)$, para códigos de comprimento $p^{n}$, sabemos que $\left\{e_{0}, \ldots, e_{n}\right\}$ é o conjunto de idempotentes primitivos ortogonais em $R G$. Quando tomamos $|G|=2^{n}$, temos um problema, pois o grupo das unidade de $Z_{2^{n}}$, para $m \geq 2$ não é cíclico e, assim, para qualquer $|\bar{R}|=q$, não teríamos a condição necessária dita anteriormente.

Agora, estamos interessados na construção de códigos que possuam distância de Hamming máxima. Para isso, tomaremos o comprimento $2^{n}$ e iremos focar nos códigos gerados pelo idempotente $e_{1}$.

No teorema a seguir provaremos que $e_{1}$ é um idempotente primitivo.

Teorema 3.3.1 Se $G=<g>$ é um grupo cíclico de ordem $|G|=2^{n}$, $R$ é um anel de cadeia tal que $|R|$ não divide 2 , então $e_{1}=\widehat{G}_{1}-\widehat{G}_{0}$ é um idempotente primitivo.

Prova: Como $e_{1}=\widehat{G}_{1}-\widehat{G}_{0}$, temos $\widehat{G}_{1}=e_{1}+\widehat{G}_{0}$ e daí $e_{1} \cdot \widehat{G}_{0}=\left(\widehat{G}_{1}-\widehat{G}_{0}\right) \cdot \widehat{G}_{0}=\widehat{G}_{0}-\widehat{G}_{0}=0$. Logo, $R G \widehat{G}_{1}=R G e_{1} \oplus R G \widehat{G}_{0}$ e, com isso, temos

$$
\left|R G \widehat{G}_{1}\right|=\left|R G e_{1}\right| \cdot\left|R G \widehat{G}_{0}\right|
$$

Assim,

$$
\left|R G e_{1}\right|=\frac{\left|R G \widehat{G}_{1}\right|}{\left|R G \widehat{G}_{0}\right|}=\frac{|R|^{\frac{|G|}{\left|G_{1}\right|}}}{|R|^{|G|}}=\frac{|R|^{2^{1}}}{|R|}=|R| .
$$

Como $\left|R G e_{1}\right|=|R|$, então $R G e_{1} \cong R$. Como $R$ é anel local, temos que $R$ é indecomponível. Assim, $e_{1}$ é um idempotente primitivo, como queríamos demonstrar.

Neste caso, como $|G|=2^{n}$, que é o comprimento do código e $w\left(R G e_{1}\right)=2 \cdot 2^{n-1}=2^{n}$, e pelo Teorema 3.3.1, $\left|R G e_{1}\right|=|R|$, o código gerado por $e_{1}$ têm distância de Hamming máxima. 


\subsubsection{Resultados Sobre Códigos MDS de comprimento $p^{n}$}

Consideremos agora $G$ um grupo cíclico de ordem $p^{n} \operatorname{com} p>2$ e $|R|=q^{k}$, com $q \nmid|G|$ tal que $o(|\bar{R}|)=\phi\left(p^{n}\right)$ em $U\left(\mathbb{Z}_{p^{n}}\right)$. Apresentaremos agora dois resultados sobre códigos MDS.

Teorema 3.3.2 Considere $R$ e $G$ nas condições da Hipótese A. O código gerado por $R G e_{i}$ onde $i>\frac{n+1}{2} n \tilde{a} o$ é $M D S$.

Prova: Como $i>\frac{n+1}{2}$, temos que $-n+2 i-1>0$. Como $p>2$, então $p^{(-n+2 i-1)}>2$, e daí $p^{i-1}>2 p^{n-i}$. Como $p^{n} \geq p^{i}$, temos $p^{n}+p^{i-1}>p^{i}+2 p^{n-i}, \log \mathrm{O}$

$$
p^{n}-p^{i}+p^{i-1}-2 p^{n-i}>0
$$

e, assim, $p^{n}-p^{i}+p^{i-1}-2 p^{n-i}+1>0$ o que resulta

$$
p^{n}-p^{i}+p^{i-1}+1>2 p^{n-i}
$$

onde $2 \cdot p^{n-i}=w\left(R G e_{i}\right)$.

Teorema 3.3.3 $S e C=R G e_{0} \oplus R G e_{1} \oplus R G e_{2} \oplus \ldots \oplus R G e_{k}$, então $C$ não é um código $M D S$.

Prova: Se $C=R G e_{0} \oplus R G e_{1} \oplus R G e_{2} \oplus \ldots \oplus R G e_{k}$, então $w(C)=2\left|G_{k}\right|=2 p^{n-k}$. Calculando o limitante 3.2, temos que

$$
d_{H}(C) \leq p^{n}+\log _{|R|}(|\bar{R}|)^{t\left(\left(p^{1}-p^{0}\right)+\left(p^{2}-p^{1}\right)+\left(p^{3}-p^{2}\right)+\ldots+\left(p^{k}-p^{k-1}\right)+1\right)}+1
$$

Desenvolvendo a desigualdade acima, temos

$$
d_{H}(C) \leq p^{n}-p^{k}+1=p^{k}\left(p^{n-k}+1\right)+1
$$


Como $p^{k}>2$, temos $p^{k}\left(p^{n-k}+1\right)+1>2 p^{n-k}$. Portanto, $C$ não é MDS.

Uma pergunta interessante é: existem códigos MDS da forma $R G e_{i}$, onde $G$ é um grupo cíclico de ordem $p^{n}$ ( $p$ primo ímpar) e $R$ um anel de cadeia? Esta pergunta ainda não possui uma resposta concreta, mas devido a algumas simulações computacionais, suspeitamos que os únicos códigos MDS da forma $R G e_{i}$ onde $G$ é um grupo cíclico de ordem $p^{n}$ sejam os triviais. 


\section{CAPÍTULO 4}

\section{Códigos sobre Anéis de Cadeia de}

\section{Comprimento $2 p^{n}$}

No Capítulo 3 calculamos o peso de todos os códigos cíclicos de comprimento $p^{n}$ sobre anéis de cadeia. Neste capítulo, calcularemos o peso de todos os possíveis códigos cíclicos sobre anéis de cadeia de comprimento $2 p^{n}$ e caracterizaremos todos os códigos livres de comprimento $2 p^{n}$, exibindo uma base para estes códigos.

A seguir, descreveremos uma hipótese central que será utilizada ao longo deste capítulo.

\section{Hipótese $B$}

Sejam $R$ um anel de cadeia finito, comutativo com unidade, tal que $|R|=q^{k}, M=\langle a\rangle$ o ideal maximal de $R,|\bar{R}|=q^{l}$, com $k=l t$, onde $t$ é o indice de nilpotência de a e $G$ um grupo cíclico com gerador $g_{0}$, de ordem $2 p^{n}$, com p primo ímpar, tal que o $(|\bar{R}|)=\phi\left(p^{n}\right)$ em $U\left(\mathbb{Z}_{2 p^{n}}\right)$, onde $q \nmid 2 p^{n}, G_{i}=<g_{0}^{p^{i}}>e e_{i}=\widehat{G}_{i}-\widehat{G}_{i-1}$. 


\subsection{Peso Mínimo de Códigos de Comprimento $2 p^{n}$}

Nesta seção, calcularemos o peso de todos os códigos cíclicos de comprimento $2 p^{n}$ sobre anéis de cadeia.

Em [15], Ferraz e Polcino Milies provaram o seguinte teorema:

Teorema 4.1.1 ( [15], Teorema 3.2) Sejam $K$ um corpo com q elementos e $A$ um grupo cíclico de ordem $2 p^{n}$, p um primo impar tal que o $(q)=\phi\left(p^{n}\right)$ em $U\left(Z_{2 p^{n}}\right)$. Escreva $A=B \times G$ onde $G$ é um p-subgrupo de Sylow e $B=\{1, d\}$ é um 2-subgrupo de Sylow. Se $e_{i}, 0 \leq i \leq n$, denota um idempotente primitivo de $K G$, então os idempotentes primitivos de $K A$ são

$$
\frac{1+d}{2} e_{i} \quad e \quad \frac{1-d}{2} e_{i}, \quad 0 \leq i \leq n
$$

Considere $K=\frac{R}{M}$. Fazendo o levantamento dos idempotentes de $K$ para $R$, temos que

$$
\left\{\frac{1+d}{2} e_{i} \quad \text { e } \quad \frac{1-d}{2} e_{i}, \quad 0 \leq i \leq n\right\}
$$

é um conjunto de idempotentes primitivos ortogonais em $R A$, onde $R$ é um anel de cadeia, $\operatorname{com}|R|=q^{k}$ e $q \nmid|A|$.

Em [15], Ferraz e Polcino Milies provaram que o peso do código $K A\left(\frac{1 \pm d}{2} e_{i}\right)$ é dado por

$$
w\left(K A\left(\frac{1 \pm d}{2} e_{i}\right)\right)=\left\{\begin{array}{l}
4\left|G_{i}\right|, \text { se } 0<i \leq n \\
|A|, \text { se } i=0
\end{array}\right.
$$

Provaremos agora um resultado semelhante para $R A\left(\frac{1 \pm d}{2} e_{i}\right)$.

Teorema 4.1.2 Considere $R$ e $G$ nas condições da Hipótese B. Seja $C=R A\left(a^{k}\left(\frac{1 \pm d}{2}\right) e_{i}\right)$, com $0 \leq k<t$. Então

$$
w(C)=\left\{\begin{array}{l}
4\left|G_{i}\right|, \text { se } 0<i \leq n \\
|A|, \text { se } i=0
\end{array}\right.
$$


Prova: Temos que $e_{i}=\widehat{G}_{i}-\widehat{G}_{i-1}$, para $i \neq 0$. Seja $\Gamma=\left\{\tau_{1}, \ldots, \tau_{n}\right\}$ um transversal de $G_{i}$ em $G$. Como $\left(a^{k}\left(\frac{1 \pm d}{2}\right) e_{i}\right) \cdot \widehat{G}_{i}=\left(a^{k}\left(\frac{1 \pm d}{2}\right) e_{i}\right)$, temos $C \subset\left(a^{k}\left(\frac{1 \pm d}{2}\right) \widehat{G}_{i}\right)$. Seja $\alpha \neq 0 \in C$, logo $\alpha$ pode ser escrito como

$$
\alpha=\left(x_{1} \tau_{1}+\ldots+x_{n} \tau_{n}\right)\left(a^{k}\left(\frac{1 \pm d}{2}\right) \widehat{G}_{i}\right)
$$

Suponhamos que exista apenas um $x_{i} a^{k} \neq 0$ tal que $\alpha=\left(x_{i} \tau_{i}\right)\left(a^{k}\left(\frac{1 \pm d}{2}\right) \widehat{G}_{i}\right)$. Então existe $\beta_{i} \in R A$ tal que

$$
\alpha=\left(x_{i} \tau_{i}\right)\left(a^{k}\left(\frac{1 \pm d}{2}\right) \widehat{G}_{i}\right)=\beta_{i} \cdot\left(a^{k}\left(\frac{1 \pm d}{2}\right) e_{i}\right)
$$

Como $\widehat{G}_{i-1} \cdot G_{i}=\widehat{G}_{i-1}$ e $e_{i} \cdot G_{i-1}=0$, pois $G_{i} \subset G_{i-1}$, temos $\left(x_{i} \tau_{i}\right)\left(a^{k}\left(\frac{1 \pm d}{2}\right) \widehat{G}_{i-1}\right)=0$. Observe que $\left(x_{i} a^{k} \tau_{i}\right) \cdot \widehat{G}_{i-1}$ e $\left(x_{i} a^{k} \tau_{i}\right) \cdot d \widehat{G}_{i-1}$ têm suportes disjuntos. Logo, $\left(x_{i} a^{k} \tau_{i}\right) \cdot \widehat{G}_{i-1}=0$ e, assim, $x_{i} a^{k} \widehat{G}_{i-1}=0$. Portanto, $x_{i} a^{k}=0$, o que contradiz nossa hipótese inicial. Logo existem pelo menos dois coeficientes diferentes de zero e, portanto, $w(C) \geq 4\left|G_{j}\right|$.

Seja $g_{0} \in G_{i-1} / G_{i}$. Temos que

$$
\begin{aligned}
\alpha & =\left(1-g_{0}\right)\left(a^{k}\left(\frac{1 \pm d}{2}\right) \widehat{G}_{i}\right) \\
& =\left(1-g_{0}\right)\left(a^{k} \frac{1 \pm d}{2}\right)\left(\widehat{G}_{i}-\widehat{G}_{i-1}+G_{i-1}\right) \\
& =\left(1-g_{0}\right)\left(a^{k} \frac{1 \pm d}{2}\right)\left(e_{i}+\widehat{G}_{i-1}\right) \\
& =\left(1-g_{0}\right)\left(a^{k} \frac{1 \pm d}{2}\right) e_{i},
\end{aligned}
$$

pois $\left(1-g_{0}\right) \cdot \widehat{G}_{i-1}=0$. Logo $\alpha=\left(1-g_{0}\right)\left(a^{k} \frac{1 \pm d}{2}\right) \widehat{G}_{i} \in C$ e, como $w(\alpha)=4\left|G_{i}\right|$, temos que $w(C) \leq 4\left|G_{i}\right|$. Portanto, $w(C)=4\left|G_{i}\right|$.

Vamos supor agora $C=\left\langle a^{k}\left(\frac{1 \pm d}{2} e_{0}\right)\right\rangle$. Provemos que $w\left(\left\langle a^{k}\left(\frac{1 \pm d}{2} e_{0}\right)\right\rangle\right)=|A|$. Seja $\alpha \neq 0 \in C$. Temos $\alpha=\sum_{g \in G} x_{g} d^{\zeta_{g}} g\left(a^{k}\left(\frac{1 \pm d}{2}\right) e_{0}\right)$, onde $\zeta_{g}=0$ ou $1, x_{g} \in R$. Como $g \cdot e_{o}=e_{0}=\widehat{G}$ e $d \cdot\left(\frac{1 \pm d}{2}\right)= \pm\left(\frac{1 \pm d}{2}\right)$, temos que $\alpha=\left(\sum a^{k} x_{g}^{\prime}\right)\left(\frac{1 \pm d}{2} e_{0}\right)$. Portanto, $w(C)=2|G|=|A|$.

Agora calcularemos os pesos de todos os possíveis códigos cíclicos de comprimento $2 p^{n}$ 
sobre um anel de cadeia $R$, e um grupo $G$ nas condições da Hipótese B. Iremos calcular os resultados de acordo com a soma dos geradores dos códigos. Nos teoremas que seguem consideraremos apenas o caso de somas de idempotentes da forma $a^{k_{i}}\left(\frac{1+d}{2}\right) e_{i}$, sendo a demonstração análoga no caso $a^{k_{i}}\left(\frac{1-d}{2}\right) e_{i}$.

Teorema 4.1.3 Considere $R$ e $G$ nas condições da Hipótese B. Se o código $C$ é da forma $C=<a^{k_{i_{1}}}\left(\frac{1+d}{2}\right) e_{i_{1}}>\oplus \ldots \oplus<a^{k_{i_{l}}}\left(\frac{1+d}{2}\right) e_{i_{l}}>$, onde $0 \leq k_{i_{j}}<t, 1 \leq j \leq l$ e $0<i_{1}<i_{2}<$ $\ldots<i_{l}$, então $w(C)=4\left|G_{i_{l}}\right|$.

\section{Prova:}

Primeiramente, observe que $w(C) \leq w\left(<a^{k_{i_{l}}}\left(\frac{1+d}{2}\right) e_{i_{l}}>\right)=4\left|G_{i_{l}}\right|$.

Como $G_{i_{l}} \subset G_{j}$, para $j<i_{l}$, temos $\widehat{G}_{i_{l}} \cdot e_{j}=e_{j}$. Logo $C \subset<a^{k}\left(\frac{1+d}{2}\right) \widehat{G}_{i_{l}}>$, onde $k=\min \left\{k_{i_{1}}, \ldots, k_{i_{l}}\right\}$. Seja $\Gamma=\left\{\tau_{1}, \ldots, \tau_{n}\right\}$ um transversal de $G_{i_{l}}$ em $G$ e $\alpha \in C$. Então podemos escrever

$$
\alpha=\left(x_{1} \tau_{1}+\ldots+x_{n} \tau_{n}\right)\left(a^{k}\left(\frac{1+d}{2}\right) \widehat{G}_{i_{l}}\right)
$$

onde $x_{i} \in R$. Suponhamos agora que exista apenas um coeficiente $x$, tal que $x a^{k} \neq 0$. Então, podemos escrever $\alpha$ como

$$
\alpha=x \tau\left(a^{k}\left(\frac{1+d}{2}\right) \widehat{G}_{i_{l}}\right)
$$

onde $\tau \in \Gamma$. Como $C=<a^{k_{i_{1}}}\left(\frac{1+d}{2}\right) e_{i_{1}}>\oplus \ldots \oplus<a^{k_{i_{l}}}\left(\frac{1+d}{2}\right) e_{i_{l}}>$, existem $\beta_{1}, \ldots, \beta_{l} \in R A$ tais que

$$
\alpha=\beta_{1} a^{k_{i_{1}}}\left(\frac{1+d}{2}\right) e_{i_{1}}+\ldots+\beta_{l} a^{k_{i_{l}}}\left(\frac{1+d}{2}\right) e_{i_{l}}=x_{1} \tau_{1}\left(a^{k}\left(\frac{1+d}{2}\right) \widehat{G}_{i_{l}}\right) .
$$

Como $G_{i_{1}-1} \supset G_{j}$, para $i_{1} \leq j \leq i_{l}$, temos $\widehat{G}_{i_{1}-1} \cdot e_{j}=0$. Logo, $x \tau\left(a^{k}\left(\frac{1+d}{2}\right) \widehat{G}_{i_{1}-1}\right)=0$. Como $x \tau\left(a^{k} \widehat{G}_{i_{1}-1}\right)$ e $x \tau\left(a^{k} d \widehat{G}_{i_{1}-1}\right)$ têm suportes disjuntos, temos que $x \tau\left(a^{k} \widehat{G}_{i_{1}-1}\right)=0$ e daí, $x a^{k}=0$, o que contradiz nossa hipótese original. Logo $w(\alpha) \geq 4\left|G_{i_{l}}\right|$ e, portanto, $w(C) \geq 4\left|G_{i_{l}}\right|$. 
Com isso, temos $w(C)=4\left|G_{i_{l}}\right|$.

Teorema 4.1.4 Considere $R$ e $G$ nas condições da Hipótese B. Se o código $C$ é da forma $C=<a^{k_{0}}\left(\frac{1+d}{2}\right) e_{0}>\oplus<a^{k_{1}}\left(\frac{1+d}{2}\right) e_{1}>\oplus \ldots \oplus<a^{k_{l}}\left(\frac{1+d}{2}\right) e_{l}>$, onde $0 \leq k_{j},<t, 0 \leq j \leq l$, então $w(C)=2\left|G_{l}\right|$.

Prova: Como $\left(a^{k_{i}}\left(\frac{1+d}{2}\right) e_{i}\right) \cdot\left(\frac{1+d}{2}\right) \widehat{G}_{l}=\left(a^{k}\left(\frac{1+d}{2}\right) e_{i}\right)$, para $0 \leq i \leq l$, temos $C \subset<a^{k_{i}}\left(\frac{1+d}{2}\right) \widehat{G}_{l}>, \log \mathrm{O}$

$$
w(C) \geq w\left(<a^{k_{i}}\left(\frac{1+d}{2}\right) \widehat{G}_{l}>\right)=2\left|G_{l}\right|
$$

Agora seja $k=\max \left\{k_{0}, \ldots, k_{l}\right\}$. Observe que

$$
\begin{aligned}
a^{k}\left(\frac{1+d}{2}\right) \widehat{G}_{l} & =a^{k}\left(\frac{1+d}{2}\right)\left(\left(\widehat{G}_{l}-\widehat{G}_{l-1}\right)+\left(\widehat{G}_{l-1}+\widehat{G}_{l-2}\right)+\ldots+\left(\widehat{G}_{1}-\widehat{G}_{0}\right)+\widehat{G}_{0}\right) \\
& =a^{k}\left(\frac{1+d}{2}\right) e_{l}+a^{k}\left(\frac{1+d}{2}\right) e_{l-1}+\ldots+a^{k}\left(\frac{1+d}{2}\right) e_{2}+a^{k}\left(\frac{1+d}{2}\right) e_{1}+a^{k}\left(\frac{1+d}{2}\right) e_{0} .
\end{aligned}
$$

Portanto,

$$
\begin{aligned}
<\left(a^{k}\left(\frac{1+d}{2}\right) \widehat{G}_{l}\right)> & \subset \quad<a^{k}\left(\frac{1+d}{2}\right) e_{0}>\oplus<a^{k}\left(\frac{1+d}{2}\right) e_{1}>\oplus \ldots \oplus<a^{k}\left(\frac{1+d}{2}\right) e_{l}> \\
& \subset<a^{k_{0}}\left(\frac{1+d}{2}\right) e_{0}>\oplus<a^{k_{1}}\left(\frac{1+d}{2}\right) e_{1}>\oplus \ldots \oplus<a^{k_{l}}\left(\frac{1+d}{2}\right) e_{l}>=C .
\end{aligned}
$$

$\operatorname{Logo} 2\left|G_{l}\right|=w\left(<\left(a^{k}\left(\frac{1+d}{2}\right) \widehat{G}_{l}\right)>\right) \geq w(C)$. Portanto, $w(C)=2\left|G_{l}\right|$.

Teorema 4.1.5 Considere $R$ e $G$ nas condições da Hipótese B. Se o código $C$ é da forma

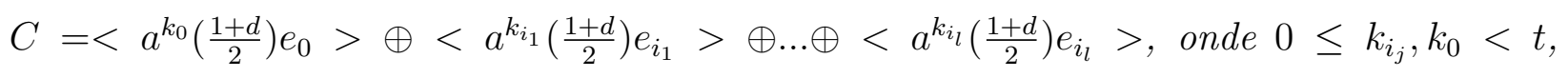
$1 \leq j \leq l$ e $\left\{i_{1}, \ldots, i_{l}\right\} \varsubsetneqq\left\{1, \ldots, i_{l}\right\}$, então $w(C)=4\left|G_{i_{l}}\right|$.

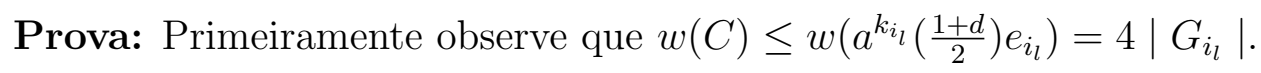

Como $G_{i_{l}} \subset G_{j}$, para $j<i_{l}$, temos $\widehat{G}_{i_{l}} \cdot e_{j}=e_{j}$. Logo $C \subset<a^{k}\left(\frac{1+d}{2}\right) \widehat{G}_{i_{l}}>$, onde $k=\max \left\{k_{i_{1}}, \ldots, k_{i_{l}}\right\}$. Seja $\Gamma=\left\{\tau_{1}, \ldots, \tau_{n}\right\}$ um transversal de $G_{i_{l}}$ em $G$ e $\alpha \in C$. Então 
podemos escrever $\alpha=\left(x_{1} \tau_{1}+\ldots+x_{n} \tau_{n}\right)\left(a^{k}\left(\frac{1+d}{2}\right) \widehat{G}_{i_{l}}\right)$, onde $x_{i} \in R$. Suponhamos agora que exista apenas um coeficiente $x$ de $\alpha$ tal que $x a^{k} \neq 0$. Então podemos escrever $\alpha$ como $\alpha=x \tau\left(a^{k}\left(\frac{1+d}{2}\right) \widehat{G}_{i_{l}}\right)$, onde $\tau \in \Gamma$. Como

$$
C=<a^{k_{0}}\left(\frac{1+d}{2}\right) e_{0}>\oplus<a^{k_{i_{1}}}\left(\frac{1+d}{2}\right) e_{i_{1}}>\oplus \ldots \oplus<a^{k_{i_{l}}}\left(\frac{1+d}{2}\right) e_{i_{l}}>,
$$

existem $\beta_{0}, \beta_{1}, \ldots, \beta_{l}$, tais que

$$
\alpha=x \tau\left(a^{k}\left(\frac{1+d}{2}\right) \widehat{G}_{i_{l}}\right)=\beta_{0} a^{k_{0}}\left(\frac{1+d}{2}\right) e_{0}+\beta_{1} a^{k_{i_{1}}}\left(\frac{1+d}{2}\right) e_{i_{1}}+\ldots+\beta_{l} a^{k_{i_{l}}}\left(\frac{1+d}{2}\right) e_{i_{l}} .
$$

Como $\left\{i_{1}, \ldots, i_{l}\right\} \varsubsetneqq\left\{1, \ldots, i_{l}\right\}$, existe pelo menos um $i_{r} \in\left\{1, \ldots, i_{l}\right\}$ tal que o idempotente $\left(\frac{1+d}{2}\right) e_{i_{r}}$ não está na soma inicial. Considere $i_{r}$ o menor número que não está em $\left\{i_{1}, \ldots, i_{l}\right\}$ e está em $\left\{1, \ldots, i_{l}\right\}$. Multiplicando ambos os lados da igualdade por $\widehat{G}_{i_{r}}$, temos

$$
\alpha=x \tau\left(a^{k}\left(\frac{1+d}{2}\right) \widehat{G}_{i_{r}}\right)=\beta_{0} a^{k_{0}}\left(\frac{1+d}{2}\right) e_{0}+\beta_{1} a^{k_{i_{1}}}\left(\frac{1+d}{2}\right) e_{i_{1}}+\ldots+\beta_{l} a^{k_{i_{r}-1}}\left(\frac{1+d}{2}\right) e_{i_{r}-1} .
$$

Como o peso do ideal $<a^{k_{0}}\left(\frac{1+d}{2}\right) e_{0}>\oplus<a^{k_{1}}\left(\frac{1+d}{2}\right) e_{i_{1}}>\oplus<a^{k_{2}}\left(\frac{1+d}{2}\right) e_{i_{2}}>\oplus \ldots \oplus$

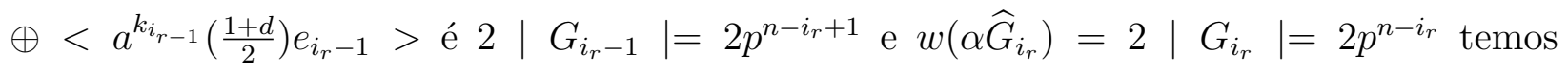
uma contradição. Com isso, temos $w(C) \geq 4\left|G_{i_{l}}\right|$ e assim, $w(C)=4\left|G_{i_{l}}\right|$.

Agora iremos calcular o peso mínimo dos códigos que sejam somas de ideais do tipo $<\left(a^{k_{i}}\left(\frac{1+d}{2}\right) e_{i}\right)>\mathrm{e}<\left(a^{k_{j}}\left(\frac{1-d}{2}\right) e_{j}\right)>$. Como o cálculo para estes códigos envolvem várias técnicas diferentes para os diferentes tipos de somas, calcularemos inicialmente o peso mínimo dos códigos que sejam somas de dois ideais apenas e depois generalizaremos estes cálculos para uma soma finita destes ideais.

Teorema 4.1.6 Considere $R$ e $G$ nas condições da Hipótese B. Se o código $C$ é da forma $C=<a^{k_{i}}\left(\frac{1+d}{2}\right) e_{i}>\oplus<a^{k_{j}}\left(\frac{1-d}{2}\right) e_{j}>$, onde $0<i<j \leq n, 0 \leq k_{i}, k_{j}<t$, então $w(C)=4\left|G_{j}\right|$.

Prova: Como $<a^{k_{j}}\left(\frac{1-d}{2}\right) e_{j}>\subset C$, temos $w(C) \leq w\left(<a^{k_{j}}\left(\frac{1-d}{2}\right) e_{j}>\right)=4\left|G_{j}\right|$. Além 
$\operatorname{disso}, \widehat{G}_{j} \cdot e_{i}=e_{i}$ e $\widehat{G}_{j} \cdot e_{j}=e_{j} . \operatorname{Logo}$

$$
C \subset<a^{k}\left(\frac{1+d}{2}\right) \widehat{G}_{j}>\oplus<a^{k}\left(\frac{1-d}{2}\right) \widehat{G}_{j}>=<a^{k} \widehat{G}_{j}>
$$

onde $k=\min \left\{k_{i}, k_{j}\right\}$. Seja $\Gamma=\left\{\tau_{1}, \ldots, \tau_{n}\right\}$ um transversal de $G_{j}$ em $G$ e $\alpha \neq 0 \in C$. Podemos escrever $\alpha=\left(x_{1} \tau_{1}+\ldots+x_{n} \tau_{n}+x_{1}^{\prime} d \tau_{1}+\ldots+x_{n}^{\prime} d \tau_{n}\right) a^{k} \widehat{G}_{j}$, onde $x_{l}$ e $x_{l}^{\prime} \in R$. Suponha que exista apenas um coeficiente $y$ de $\alpha$ tal que $y a^{k} \neq 0$. Então $\alpha$ é da forma $\alpha=\left(y a^{k} d^{\zeta_{\tau}} \tau\right) \widehat{G}_{j}$, onde $\zeta_{\tau}=0$ ou 1 e $\tau \in \Gamma$. Logo existem $\beta_{i}, \beta_{j} \in R A$ tais que

$$
\alpha=\left(y a^{k} d^{\zeta_{\tau}} \tau\right) \widehat{G}_{j}=\beta_{i} a^{k_{i}}\left(\frac{1+d}{2}\right) e_{i}+\beta_{j} a^{k_{j}}\left(\frac{1-d}{2}\right) e_{j} .
$$

Multiplicando ambos os lados da igualdade por $\widehat{G}_{i-1}$, temos $\left(y a^{k} d^{\zeta_{\tau}} \tau\right) \widehat{G}_{i-1}=0$. Logo, $y a^{k}=0$, o que é uma contradição.

Suponhamos agora que existam apenas dois coeficientes $y_{1}, y_{2}$ de $\alpha$ tais que $y_{1} a^{k} \neq 0$ e $y_{2} a^{k} \neq 0$. Assim, podemos escrever

$$
\alpha=\left(y_{1} d^{\zeta_{\tau}} \tau+y_{2} d^{\zeta_{\tau^{\prime}}} \tau^{\prime}\right) a^{k} \widehat{G}_{j}=\beta_{i} a^{k_{i}}\left(\frac{1+d}{2}\right) e_{i}+\beta_{j} a^{k_{j}}\left(\frac{1-d}{2}\right) e_{j}
$$

onde $\zeta_{\tau}, \zeta_{\tau^{\prime}}=0$ ou 1 e $\tau$ e $\tau^{\prime} \in \Gamma$. Suponhamos agora $\zeta_{\tau} \neq \zeta_{\tau^{\prime}}$. Multiplicando ambos os lados por $\widehat{G}_{i-1}$, temos $\left(y_{1} d^{\zeta_{\tau}} \tau+y_{2} d_{\tau^{\prime}} \tau^{\prime}\right) a^{k} \widehat{G}_{i-1}=0$. Como $\zeta_{\tau} \neq \zeta_{\tau^{\prime}}$, então as palavras $\left(y_{1} d^{\zeta_{\tau}} \tau\right) a^{k} \widehat{G}_{i-1}$ e $\left(y_{2} d^{\zeta_{\tau^{\prime}}} \tau^{\prime}\right) a^{k} \widehat{G}_{i-1}$, têm suportes disjuntos. Portanto,

$$
\left(y_{1} d^{\zeta \tau} \tau\right) a^{k} \widehat{G}_{i-1}=\left(y_{2} d^{\zeta_{\tau^{\prime}}} \tau^{\prime}\right) a^{k} \widehat{G}_{i-1}=0
$$

e assim, $y_{1}=y_{2}=0$, o que contradiz nossa hipótese. Suponhamos agora sem perda de generalidade que $\zeta_{\tau}=\zeta_{\tau^{\prime}}=0$. Assim,

$$
\alpha=\left(y_{1} \tau+y_{2} \tau^{\prime}\right) a^{k} \widehat{G}_{j}=\beta_{i} a^{k_{i}}\left(\frac{1+d}{2}\right) e_{i}+\beta_{j} a^{k_{j}}\left(\frac{1-d}{2}\right) e_{j}
$$


Multiplicando ambos os lados da igualdade 4.1 por $\left(\frac{1-d}{2}\right) \widehat{G}_{j-1}$, temos

$$
\left(y_{1} \tau+y_{2} \tau^{\prime}\right) a^{k}\left(\frac{1-d}{2}\right) \widehat{G}_{j-1}=0
$$

Desenvolvendo os termos da igualdade acima, temos

$$
\left(y_{1} \tau+y_{2} \tau^{\prime}\right) a^{k} \widehat{G}_{j-1}-\left(y_{1} d \tau_{1}+y_{2} d \tau_{2}\right) a^{k} \widehat{G}_{j-1}=0
$$

Como as palavras $\left(y_{1} \tau+y_{2} \tau^{\prime}\right) a^{k} \widehat{G}_{j-1}$ e $\left(y_{1} d \tau+y_{2} d \tau^{\prime}\right) a^{k} \widehat{G}_{j-1}$ têm suportes disjuntos, temos $\left(y_{1} \tau+y_{2} \tau^{\prime}\right) a^{k} \widehat{G}_{j-1}=0$. Agora, multiplicando a equação 4.1 por $\widehat{G}_{j-1}$, temos

$$
0=\left(y_{1} \tau+y_{2} \tau^{\prime}\right) a^{k} \widehat{G}_{j-1}=\beta_{i} a^{k_{i}}\left(\frac{1+d}{2}\right) e_{i}
$$

Portanto, $\alpha \in<a^{k_{j}}\left(\frac{1-d}{2}\right) e_{j}>$. Mas o peso de $\alpha$ é

$$
w(\alpha)=2\left|G_{j}\right| \quad e \quad w\left(<a^{k_{j}}\left(\frac{1-d}{2}\right) e_{j}>\right)=4\left|G_{j}\right|
$$

o que é um absurdo, pois contradiz a condição da minimalidade do peso de $<a^{k_{j}}\left(\frac{1-d}{2}\right) e_{j}>$.

Suponhamos agora que existam apenas três coeficientes $y_{1}, y_{2}, y_{3}$ de $\alpha$, tais que $y_{1} a^{k} \neq 0, y_{2} a^{k} \neq 0, y_{3} a^{k} \neq 0$. Então, podemos escrever

$$
\alpha=\left(y_{1} d^{\zeta_{\tau}} \tau+y_{2} d^{\zeta^{\prime}} \tau^{\prime}+y_{3} d^{\zeta_{\tau^{\prime \prime}}} \tau^{\prime \prime}\right) a^{k} \widehat{G}_{j}=\beta_{i} a^{k_{i}}\left(\frac{1+d}{2}\right) e_{i}+\beta_{j} a^{k_{j}}\left(\frac{1-d}{2}\right) e_{j},
$$

onde $\zeta_{\tau}, \zeta_{\tau^{\prime}}, \zeta_{\tau^{\prime \prime}}=0$ ou 1 e $\tau, \tau^{\prime}$ e $\tau^{\prime \prime} \in \Gamma$. Suponhamos que $\zeta_{\tau}=\zeta_{\tau^{\prime}} \neq \zeta_{\tau^{\prime \prime}}$. Multiplicando a equação acima por $\widehat{G}_{i-1}$, temos

$$
\left(y_{1} d^{\zeta_{\tau}} \tau+y_{2} d^{\zeta_{\tau^{\prime}}} \tau^{\prime}+y_{3} d_{\tau^{\prime \prime}}^{\zeta^{\prime \prime}}\right) a^{k} \widehat{G}_{i-1}=0
$$

Como $\left(y_{1} d^{\zeta_{\tau}} \tau+y_{2} d_{\tau^{\prime}}^{\zeta^{\prime}}\right) a^{k} G_{j-1}$ e $\quad\left(y_{3} d_{\tau^{\prime \prime}} \tau^{\prime \prime}\right) a^{k} \widehat{G}_{i-1}$ têm suportes disjuntos, temos 
$y_{3} a^{k}=0$, o que contradiz nossa hipótese inicial. Suponhamos então, sem perda de generalidade, que $\zeta_{\tau}=\zeta_{\tau^{\prime}}=\zeta_{\tau^{\prime \prime}}=0$. Assim,

$$
\alpha=\left(y_{1} \tau+y_{2} \tau^{\prime}+y_{3} \tau^{\prime \prime}\right) a^{k} \widehat{G}_{j}=\beta_{i} a^{k_{i}}\left(\frac{1+d}{2}\right) e_{i}+\beta_{j} a^{k_{j}}\left(\frac{1-d}{2}\right) e_{j}
$$

Multiplicando ambos os lados da equação acima por $\left(\frac{1-d}{2}\right) \widehat{G}_{j-1}$, temos

$$
\left(y_{1} \tau+y_{2} \tau^{\prime}+y_{3} \tau^{\prime \prime}\right) a^{k}\left(\frac{1-d}{2}\right) \widehat{G}_{j-1}=0
$$

Desenvolvendo os termos da igualdade acima, temos

$$
\left(y_{1} \tau+y_{2} \tau^{\prime}+y_{3} \tau^{\prime \prime}\right) a^{k} \widehat{G}_{j-1}-\left(y_{1} \tau+y_{2} \tau^{\prime}+y_{3} \tau^{\prime \prime}\right) a^{k} d \widehat{G}_{j-1}=0
$$

Como as palavras $\left(y_{1} \tau+y_{2} \tau^{\prime}+y_{3} \tau^{\prime \prime}\right) a^{k} \widehat{G}_{j-1}$ e $\left(y_{1} \tau+y_{2} \tau^{\prime}+y_{3} \tau^{\prime \prime}\right) a^{k} d \widehat{G}_{j-1}$ têm suportes disjuntos, temos $\left(y_{1} \tau+y_{2} \tau^{\prime}+y_{3} \tau^{\prime \prime}\right) a^{k} \widehat{G}_{j-1}=0$.

Multiplicando a equação 4.2 por $\widehat{G}_{j-1}$, temos

$$
0=\left(y_{1} \tau+y_{2} \tau^{\prime}+y_{3} \tau^{\prime \prime}\right) a^{k} \widehat{G}_{j-1}=\beta_{i} a^{k_{i}}\left(\frac{1+d}{2}\right) e_{i}=0
$$

Portanto, $\alpha \in<a^{k_{j}}\left(\frac{1-d}{2}\right) e_{j}>$. Mas o peso de $\alpha$ é

$$
w(\alpha)=3\left|G_{j}\right| \quad e \quad w\left(<a^{k_{j}}\left(\frac{1-d}{2}\right) e_{j}>\right)=4\left|G_{j}\right|
$$

o que contradiz a minimalidade do peso de $\left\langle a^{k_{j}}\left(\frac{1-d}{2}\right) e_{j}\right\rangle$.

Portanto, $w(C) \geq 4\left|G_{j}\right|$ e, assim, temos $w(C)=4\left|G_{j}\right|$.

Teorema 4.1.7 Considere $R$ e $G$ nas condições da Hipótese B. Se o código $C$ é da forma $C=<a^{k_{0}}\left(\frac{1+d}{2}\right) e_{0}>\oplus<a^{k_{j}}\left(\frac{1-d}{2}\right) e_{j}>$, onde $1<j \leq n, 0 \leq k_{0}, k_{j}<t$, então $w(C)=4\left|G_{j}\right|$. 
Prova: Primeiramente, temos $w(C) \leq w\left(<a^{k_{j}}\left(\frac{1-d}{2}\right) e_{j}>\right)=4\left|G_{j}\right|$.

Observe que $C \subset<a^{k} \widehat{G}_{j}>$, onde $k=\min \left\{k_{0}, k_{j}\right\}$. Seja $\Gamma=\left\{\tau_{1}, \ldots, \tau_{n}\right\}$ um transversal de $G_{j}$ em $G$ e $0 \neq \alpha \in C$. Assim, $\alpha=\left(x_{1} \tau_{1}+\ldots+x_{n}^{\prime} \tau_{n}+x_{1}^{\prime} d \tau_{1}+\ldots+x_{n}^{\prime} d \tau_{n}\right) a^{k} \widehat{G}_{j}$.

Suponhamos que exista apenas um coeficiente $y$ de $\alpha$ tal que $y a^{k} \neq 0$. Assim, podemos escrever $\alpha$ como

$$
\alpha=\left(y d^{\zeta_{\tau}} \tau\right) a^{k} \widehat{G}_{j}
$$

onde $\zeta_{\tau}=0$ ou 1. Logo, existem $\alpha_{1}, \alpha_{2} \in R A$ tais que

$$
\left(y d^{\zeta_{\tau}} \tau\right) a^{k} \widehat{G}_{j}=\alpha_{1} a^{k_{0}}\left(\frac{1+d}{2}\right) e_{0}+\alpha_{2} a^{k_{j}}\left(\frac{1-d}{2}\right) e_{j}
$$

Multiplicando a igualdade acima por $\widehat{G}_{j-1}$, temos

$$
\left(y d^{\zeta_{\tau}} \tau\right) a^{k} \widehat{G}_{j-1}=\alpha_{1} a^{k_{0}}\left(\frac{1+d}{2}\right) e_{0}
$$

Daí, $\left(y d^{\zeta_{\tau}} \tau\right) a^{k} \widehat{G}_{j-1}=0$, pois independente do valor de $\zeta_{\tau}$ na igualdade 4.3 , teríamos $\alpha_{1} a^{k_{0}} \widehat{G}=0$ ou $\alpha_{1} a^{k_{0}} d \widehat{G}=0$ e portanto $y a^{k}=0$.

Suponhamos agora que existam apenas dois coeficientes $y_{1}, y_{2}$ de $\alpha$ tais que $y_{1} a^{k} \neq 0$, $y_{2} a^{k} \neq 0$. Logo existem $\alpha_{1}, \alpha_{2} \in R A$ tais que

$$
\alpha=\left(y_{1} d^{\zeta_{\tau}} \tau+y_{2} d^{\zeta_{\tau^{\prime}}} \tau^{\prime}\right) a^{k} \widehat{G}_{j}=\alpha_{1} a^{k_{0}}\left(\frac{1+d}{2}\right) e_{0}+\alpha_{2} a^{k_{j}}\left(\frac{1-d}{2}\right) e_{j},
$$

onde $\zeta_{\tau}, \zeta_{\tau^{\prime}}=0$ ou 1 e $\tau$ e $\tau^{\prime} \in \Gamma$. Temos $\alpha_{1}=\sum_{g \in G} x_{g} d^{\zeta_{g}} g$, onde $\zeta_{g}=0$ ou 1 . Logo $\left.\alpha_{1} \cdot\left(a^{k_{0}} \frac{1+d}{2}\right) e_{0}\right)=\left(\sum x_{g}\right) a^{k_{0}}\left(\frac{1+d}{2}\right) e_{0}=\left(\sum x_{g}\right) a^{k_{0}}\left(\frac{1+d}{2}\right) \widehat{G}$. Assim,

$$
\alpha=\left(y_{1} d^{\zeta_{\tau}} \tau+y_{2} d_{\tau^{\prime}}^{\zeta^{\prime}}\right) a^{k} \widehat{G}_{j-1}=\left(\sum x_{g}\right) a^{k_{0}}\left(\frac{1+d}{2}\right) \widehat{G}
$$

Suponhamos, sem perda de generalidade, $\zeta_{\tau} \neq \zeta_{\tau^{\prime}}$ e $\zeta_{\tau}=0$. Como 
$\left|\tau G_{j-1}\right|=\left|\tau^{\prime} G_{j-1}\right|=\left|G_{j-1}\right|<|G|$, pois $j>1$, temos $\left(\sum x_{g} a^{k_{0}}\right)=0$, pois

$$
y_{1} \tau a^{k} G_{j-1}=\frac{1}{2}\left(\sum x_{g} a^{k_{0}}\right) \widehat{G} \text { e } y_{2} d \tau^{\prime} a^{k} G_{j-1}=\frac{1}{2}\left(\sum x_{g} a^{k_{0}}\right) d \widehat{G} .
$$

Assim, $y_{1} a^{k}=y_{2} a^{k}=0$, o que contradiz nossa hipótese.

Suponhamos agora $\zeta_{\tau}=\zeta_{\tau^{\prime}}=0$. Assim,

$$
\alpha=\left(y_{1} \tau+y_{2} \tau^{\prime}\right) a^{k} \widehat{G}_{j}=\alpha_{1} a^{k_{0}}\left(\frac{1+d}{2}\right) e_{0}+\alpha_{2} a^{k_{j}}\left(\frac{1-d}{2}\right) e_{j}
$$

Multiplicando a igualdade acima por $\widehat{G}_{j-1}$, temos

$$
\left(y_{1} \tau+y_{2} \tau^{\prime}\right) a^{k} \widehat{G}_{j-1}=\alpha_{1} a^{k_{0}}\left(\frac{1+d}{2}\right) e_{0}
$$

Novamente, podemos escrever $\alpha_{1} \cdot a^{k_{0}}\left(\frac{1+d}{2}\right) e_{0}=\left(\sum x_{g}\right) a^{k_{0}}\left(\frac{1+d}{2}\right) e_{0}=\left(\sum x_{g}\right) a^{k_{0}}\left(\frac{1+d}{2}\right) \widehat{G}$ e, novamente, teríamos $\frac{1}{2}\left(\sum x_{g}\right) a^{k_{0}} d \widehat{G}=0 \mathrm{e} \operatorname{assim},\left(\sum x_{g}\right) a^{k_{0}}=0$. Portanto, teríamos $\alpha=\alpha_{2} a^{k_{j}}\left(\frac{1-d}{2}\right) e_{j}$, ou seja, $\alpha \in<a^{k_{j}}\left(\frac{1-d}{2}\right) e_{j}>$, porém, $w(\alpha)=2\left|G_{j}\right| \mathrm{e}$ $w\left(<a^{k_{j}}\left(\frac{1-d}{2}\right) e_{j}>\right)=4\left|G_{j}\right|$, o que é um absurdo.

Suponhamos agora que existam apenas três coeficientes $y_{1}, y_{2}$ e $y_{3}$ de $\alpha$ tais que $y_{1} a^{k} \neq 0$, $y_{2} a^{k} \neq 0$ e $y_{3} a^{k} \neq 0$. Logo existem $\alpha_{1}, \alpha_{2} \in R A$ tais que

$$
\alpha=\left(y_{1} d^{\zeta_{\tau}} \tau_{1}+y_{2} d^{\zeta_{\tau^{\prime}}} \tau^{\prime}+y_{3} d^{\zeta_{\tau^{\prime \prime}}} \tau^{\prime \prime}\right) a^{k} \widehat{G}_{j}=\alpha_{1} a^{k_{0}}\left(\frac{1+d}{2}\right) e_{0}+\alpha_{2} a^{k_{j}}\left(\frac{1-d}{2}\right) e_{j},
$$

onde $\zeta_{\tau}, \zeta_{\tau^{\prime}}, \zeta_{\tau^{\prime \prime}}=0$ ou 1 e $\tau, \tau^{\prime}$ e $\tau^{\prime \prime} \in \Gamma$. Temos $\alpha_{1}=\sum_{g \in G} x_{g} d^{\zeta_{g}} g$, onde $\zeta_{g}=0$ ou 1. Logo $\left.\alpha_{1} \cdot\left(a^{k_{0}} \frac{1+d}{2}\right) e_{0}\right)=\left(\sum x_{g}\right) a^{k_{0}}\left(\frac{1+d}{2}\right) e_{0}=\left(\sum x_{g}\right) a^{k_{0}}\left(\frac{1+d}{2}\right) \widehat{G}$. Assim,

$$
\left(y_{1} d^{\zeta_{\tau}} \tau_{1}+y_{2} d_{\tau^{\prime}}^{\zeta^{\prime}}+y_{3} d_{\tau^{\prime \prime}}^{\zeta^{\prime \prime}}\right) a^{k} \widehat{G}_{j-1}=\left(\sum x_{g}\right) a^{k_{0}}\left(\frac{1+d}{2}\right) \widehat{G}
$$

Se $\zeta_{\tau}=\zeta_{\tau^{\prime}} \neq \zeta_{\tau^{\prime \prime}}$, teríamos $y_{3} a^{k}=0$, pois se $\zeta_{\tau^{\prime \prime}}=0($ ou 1$)$, então $y_{3} \zeta_{\tau^{\prime \prime}} a^{k} \widehat{G}_{j-1}=\frac{1}{2}\left(\sum x_{g}\right) \widehat{G}$, onde $\left|\tau_{3} G_{j-1}\right|=\left|G_{j-1}\right|<|G|$. 
Vamos considerar agora, sem perda de generalidade, $\zeta_{\tau}=\zeta_{\tau^{\prime}}=\zeta_{\tau^{\prime \prime}}=0$. Assim,

$$
\left(y_{1} \tau+y_{2} \tau^{\prime}+y_{3} \tau^{\prime \prime}\right) a^{k} \widehat{G}_{j-1}=\left(\sum x_{g}\right) a^{k_{0}}\left(\frac{1+d}{2}\right) \widehat{G}
$$

Daí, $\left(\sum x_{g}\right) a^{k_{0}}\left(\frac{1}{2}\right) d \widehat{G}=0$ e assim, $\left(\sum x_{g}\right) a^{k_{0}}=0$. Logo $\alpha \in<a^{k_{j}}\left(\frac{1-d}{2}\right) e_{j}>$, mas $w(\alpha)=3\left|G_{j}\right|$ e $w\left(<a^{k_{j}}\left(\frac{1-d}{2}\right) e_{j}>\right)=4\left|G_{j}\right|$, o que é um absurdo.

Assim, $w(C) \geq 4\left|G_{j}\right|$ e portanto, $w(C)=4\left|G_{j}\right|$.

Teorema 4.1.8 Considere $R$ e $G$ nas condições da Hipótese B, onde $p>3$. Se o código $C$ é da forma $C=<a^{k_{0}}\left(\frac{1+d}{2}\right) e_{0}>\oplus<a^{k_{1}}\left(\frac{1-d}{2}\right) e_{1}>$, onde $1<j \leq n, 0 \leq k_{0}, k_{1}<t$, então $w(C)=4\left|G_{1}\right|$.

Prova: Temos $w(C) \leq w\left(<a^{k_{1}}\left(\frac{1-d}{2}\right) e_{1}>\right)=4\left|G_{1}\right|$. Provemos agora que $w(C) \geq 4\left|G_{1}\right|$.

Observe que $C \subset<a^{k} \widehat{G}_{1}>$, onde $k=\min \left\{k_{0}, k_{1}\right\}$. Sejam $\Gamma=\left\{\tau_{1}, \ldots, \tau_{p}\right\}$ um transversal de $G_{1}$ em $G$ e $\alpha \neq 0 \in C$.

Suponhamos que exista apenas um coeficiente $y$ de $\alpha$ tal que $y a^{k} \neq 0$. Então existem $\alpha_{0}$ e $\alpha_{1} \in R A$, tal que $\alpha=\left(y d^{\zeta_{\tau}} \tau\right) a^{k} \widehat{G}_{1}=\alpha_{0} a^{k_{0}}\left(\frac{1+d}{2}\right) e_{0}+\alpha_{1} a^{k_{1}}\left(\frac{1-d}{2}\right) e_{1}$, onde $\zeta_{\tau}=1$ ou 0 e $\tau \in \Gamma$.

Multiplicando a igualdade acima por $\left(\frac{1-d}{2}\right) \widehat{G}$, temos

$$
\left(y d^{\zeta_{\tau}} \tau\right) a^{k}\left(\frac{1-d}{2}\right) \widehat{G}=0
$$

Desenvolvendo, temos $\left(y d^{\zeta_{\tau}} \tau\right) a^{k} \widehat{G}-\left(y d^{\zeta_{\tau}} \tau\right) a^{k} d \widehat{G}=0$. Como as palavras $\left(y d^{\zeta_{\tau}} \tau\right) a^{k} \widehat{G}$ e $\left(y d^{\zeta_{\tau}} \tau\right) a^{k} d \widehat{G}$ têm suportes disjuntos, temos $\left(y d^{\zeta_{\tau}} \tau\right) a^{k} \widehat{G}=0$ e, portanto, $y a^{k}=0$, o que contradiz nossa hipótese inicial.

Suponhamos que existam apenas dois coeficiente $y_{1}, y_{2}$ de $\alpha$ tais que $y_{1} a^{k} \neq 0$ e $y_{2} a^{k} \neq 0$. 
Então existem $\alpha_{0}$ e $\alpha_{1} \in R A$, tais que

$$
\alpha=\left(y_{1} d^{\zeta_{\tau}} \tau+y_{2} d^{\zeta_{\tau^{\prime}}} \tau^{\prime}\right) a^{k} \widehat{G}_{1}=\alpha_{0} a^{k_{0}}\left(\frac{1+d}{2}\right) e_{0}+\alpha_{1} a^{k_{1}}\left(\frac{1-d}{2}\right) e_{1}
$$

onde $\zeta_{\tau}, \zeta_{\tau^{\prime}}=0$ ou 1 e $\tau$ e $\tau^{\prime} \in \Gamma$.

Se $\zeta_{\tau}=\zeta_{\tau^{\prime}}$, multiplicando a igualdade acima por $\widehat{G}$, temos

$$
\left(y_{1} \tau+y_{2} \tau^{\prime}\right) a^{k} \widehat{G}=\alpha_{0} a^{k_{0}}\left(\frac{1+d}{2}\right) e_{0}
$$

Como $\alpha_{0} \in R A$, então $\alpha_{0}=\sum_{g \in G} x_{g} d^{\zeta g} g$ e, assim, $\alpha_{0} a^{k_{0}}\left(\frac{1+d}{2}\right) e_{0}=\left(\sum x_{g}\right) a^{k_{0}}\left(\frac{1+d}{2}\right) \widehat{G}$. Portanto, $\left(\sum x_{g}\right) a^{k_{0}} t \widehat{G}=0$ e $\operatorname{assim}\left(\sum x_{g}\right) a^{k_{0}}=0 . \quad$ Logo $\alpha \in<a^{k_{1}}\left(\frac{1-d}{2}\right) e_{1}>$, mas $w(\alpha)=2\left|G_{1}\right|$ e $w\left(<a^{k_{1}}\left(\frac{1-d}{2}\right) e_{1}>\right)=4\left|G_{1}\right|$, o que é um absurdo.

Se $\zeta_{\tau}=0 \neq \zeta_{\tau^{\prime}}$, então

$$
\alpha=\left(y_{1} \tau+y_{2} d \tau^{\prime}\right) a^{k} \widehat{G}_{1}=\alpha_{0} a^{k_{0}}\left(\frac{1+d}{2}\right) e_{0}+\alpha_{1} a^{k_{1}}\left(\frac{1-d}{2}\right) e_{1}
$$

Multiplicando a igualdade acima por $\left(\frac{1+d}{2}\right)$, temos

$$
\left(y_{1} \tau+y_{2} d \tau^{\prime}\right)\left(\frac{1+d}{2}\right) a^{k} \widehat{G}_{1}=\left(\sum x_{g}\right) a^{k_{0}}\left(\frac{1+d}{2}\right) \widehat{G}
$$

Desenvolvendo e igualando termo a termo, temos

$$
\left(y_{1} \tau+y_{2} \tau^{\prime}\right) a^{k} \widehat{G}_{1}=\left(\sum x_{g}\right) a^{k_{0}} \widehat{G}
$$

Como estamos considerando $p \neq 3$, temos $|G| \neq\left|\tau_{1} G_{1}\right|+\left|\tau_{2} G_{1}\right|=2\left|G_{1}\right|$. Logo $\left(\sum x_{g}\right) a^{k_{0}}=0 \mathrm{e}$, assim, $\alpha \in a^{k_{1}}\left(\frac{1-d}{2}\right) e_{1}>$, mas $w(\alpha)=2\left|G_{1}\right| \mathrm{e}$ $w\left(<a^{k_{1}}\left(\frac{1-d}{2}\right) e_{1}>\right)=4\left|G_{1}\right|$, o que é um absurdo.

Suponhamos que existam apenas três coeficiente $y_{1}, y_{2}$ e $y_{3}$ de $\alpha$ tais que $y_{1} a^{k} \neq 0$, 
$y_{2} a^{k} \neq 0$ e $y_{3} a^{k} \neq 0$. Então existem $\alpha_{0}$ e $\alpha_{1} \in R A$ tais que

$$
\alpha=\left(y_{1} d^{\zeta_{\tau}} \tau+y_{2} d_{\tau^{\prime}}^{\zeta^{\prime}}+y_{3} d^{\zeta_{\tau^{\prime \prime}}} \tau^{\prime \prime}\right) a^{k} \widehat{G}_{1}=\alpha_{0} a^{k_{0}}\left(\frac{1+d}{2}\right) e_{0}+\alpha_{1} a^{k_{1}}\left(\frac{1-d}{2}\right) e_{1}
$$

onde $\zeta_{\tau}, \zeta_{\tau^{\prime}}$ e $\zeta_{\tau^{\prime \prime}}=0$ ou 1 e $\tau, \tau^{\prime}$ e $\tau^{\prime \prime} \in \Gamma$.

Se $\zeta_{\tau}=\zeta_{\tau^{\prime}}=\zeta_{\tau^{\prime \prime}}=0$, multiplicando a igualdade acima por $\widehat{G}$, temos

$$
\left(y_{1} \tau+y_{2} \tau^{\prime}+y_{3} \tau^{\prime \prime}\right) a^{k} \widehat{G}=\left(\sum x_{g}\right) a^{k_{0}}\left(\frac{1+d}{2}\right) \widehat{G}
$$

Portanto, $\left(\sum x_{g}\right) a^{k_{0}} d \widehat{G}=0$ e, assim, $\left(\sum x_{g}\right) a^{k_{0}}=0$. Logo, $\alpha \in<a^{k_{1}}\left(\frac{1-d}{2}\right) e_{1}>$, mas $w(\alpha)=3\left|G_{1}\right|$ e $w\left(<a^{k_{1}}\left(\frac{1-d}{2}\right) e_{1}>\right)=4\left|G_{1}\right|$ e daí temos $\alpha=0$.

Agora vamos supor $\zeta_{\tau}=\zeta_{\tau^{\prime}}=0$ e que $\zeta_{\tau^{\prime \prime}}=1$. Assim, $\alpha$ é dado por

$$
\alpha=\left(y_{1} \tau+y_{2} \tau^{\prime}+y_{3} d \tau^{\prime \prime}\right) a^{k} \widehat{G}_{1}=\alpha_{0} a^{k_{0}}\left(\frac{1+d}{2}\right) e_{0}+\alpha_{1} a^{k_{1}}\left(\frac{1-d}{2}\right) e_{1}
$$

Multiplicando a igualdade por $\left(\frac{1+d}{2}\right)$, temos

$$
\left(y_{1} \tau+y_{2} \tau^{\prime}+y_{3} d \tau^{\prime \prime}\right) a^{k}\left(\frac{1+d}{2}\right) \widehat{G}_{1}=\alpha_{0} a^{k_{0}}\left(\frac{1+d}{2}\right) e_{0}=\left(\sum x_{g}\right) a^{k_{0}}\left(\frac{1+d}{2}\right) \widehat{G}
$$

Da igualdade 4.4 temos $\left(y_{1} \tau+y_{2} \tau^{\prime}+y_{3} \tau^{\prime \prime}\right) a^{k} \widehat{G}_{1}=\left(\sum x_{g}\right) a^{k_{0}} \widehat{G}$. Como estamos considerando $p>3$, temos $|G| \neq\left|\tau G_{1}\right|+\left|\tau^{\prime} G_{1}\right|+\left|\tau^{\prime \prime} G_{1}\right|=3\left|G_{1}\right| . \operatorname{Logo}\left(\sum x_{g}\right) a^{k_{0}}=0$ e assim, $\alpha \in<a^{k_{1}}\left(\frac{1-d}{2}\right) e_{1}>$, mas $w(\alpha)=3\left|G_{1}\right|$ e $w\left(<a^{k_{1}}\left(\frac{1-d}{2}\right) e_{1}>\right)=4\left|G_{1}\right|$, o que é um absurdo.

Portanto, $w(C) \geq 4\left|G_{1}\right|$ e assim temos $w(C)=4\left|G_{1}\right|$.

Teorema 4.1.9 Considere $R$ e $G$ nas condições da Hipótese B. Se o código $C$ é da forma $C=<a^{k_{i}}\left(\frac{1+d}{2}\right) e_{i}>\oplus<a^{k_{j}}\left(\frac{1-d}{2}\right) e_{i}>$, onde $0<i<j \leq n, 0 \leq k_{i}, k_{j}<t$, então $w(C)=2\left|G_{j}\right|$. 
Prova: Seja $k=\min \left\{k_{i}, k_{j}\right\}$. Então

$C \subset<a^{k}\left(\frac{1+d}{2}\right) e_{i}>\oplus<a^{k}\left(\frac{1-d}{2}\right) e_{i}>\subset<a^{k}\left(\frac{1+d}{2}\right) \widehat{G}_{i}>+<a^{k}\left(\frac{1-d}{2}\right) \widehat{G}_{i}>\subset<a^{k} \widehat{G}_{i}>$.

Sejam $\Gamma=\left\{\tau_{1}, \ldots, \tau_{n}\right\}$ um transversal de $G_{i}$ em $G$ e $\alpha \in C$. Podemos escrever $\alpha$ como $\alpha=\left(x_{1} \tau_{1}+\ldots+x_{n} \tau_{n}+x_{1}^{\prime} d \tau_{1}+\ldots+x_{n}^{\prime} d \tau_{n}\right) a^{k} \widehat{G}_{i}$, onde $x_{j}$ e $x_{j}^{\prime} \in R$.

Suponhamos que exista apenas um coeficiente $y$ de $\alpha$ tal que $y a^{k} \neq 0$. Então existem $\beta_{1}$ e $\beta_{2} \in R A$ tais que

$$
\alpha=\left(y d^{\zeta_{\tau}} \tau\right) a^{k} \widehat{G}_{i}=\beta_{1} a^{k_{i}}\left(\frac{1+d}{2}\right) e_{i}+\beta_{2} a^{k_{j}}\left(\frac{1-d}{2}\right) e_{i}
$$

onde $\zeta_{\tau}=0$ ou 1 e $\tau \in \Gamma$.

Multiplicando a igualdade acima por $\widehat{G}_{i-1}$, temos $\left(y d^{\zeta_{\tau}} \tau\right) a^{k} \widehat{G}_{i-1}=0$. Assim, $y a^{k}=0$, o que contradiz nossa hipótese inicial. Portanto $w(C) \geq 2\left|G_{i}\right|$.

Agora, tomemos $g_{0} \in G_{i-1} / G_{i}$ e considere o elemento $\alpha=\left(1-g_{0}\right) a^{k} \widehat{G}_{i}$, onde $k=\max \left\{k_{i}, k_{j}\right\}$. Temos que $w(\alpha)=2\left|G_{i}\right|$. Podemos reescrever $\alpha$ como:

$$
\alpha=\left(1-g_{0}\right) a^{k} \widehat{G}_{i}=\left(1-g_{0}\right) a^{k}\left(\widehat{G}_{i-1}-\widehat{G}_{i}+\widehat{G}_{i-1}\right)=\left(1-g_{0}\right) a^{k}\left(e_{i}+\widehat{G}_{i-1}\right) .
$$

Como $\left(1-g_{0}\right) \widehat{G}_{i-1}=0$, pois $g_{0} \in G_{i-1}$, então

$$
\alpha=\left(1-g_{0}\right) a^{k} e_{i}=\left(1-g_{0}\right) a^{k}\left(\frac{1+d}{2}\right) e_{i}+\left(1-g_{0}\right) a^{k}\left(\frac{1-d}{2}\right) e_{i} \in C
$$

Logo $w(C) \leq 2\left|G_{i}\right|$ e, portanto, $w(C)=2\left|G_{i}\right|$.

Agora generalizaremos estes casos e calcularemos outros possíveis casos onde possam ocorrer somas dos idempotentes $\left(\frac{1+d}{2}\right) e_{0}$ e $\left(\frac{1-d}{2}\right) e_{0},\left(\frac{1+d}{2}\right) e_{i}$ e $\left(\frac{1-d}{2}\right) e_{i}$.

Teorema 4.1.10 Considere $R$ e $G$ nas condições da Hipótese B. Se o código $C$ é da forma $C=<a^{k_{i_{1}}}\left(\frac{1 \pm d}{2}\right) e_{i_{1}}>\oplus \ldots \oplus<a^{k_{i_{l}}}\left(\frac{1 \pm d}{2}\right) e_{i_{l}}>$, onde $0 \leq k_{i_{j}}<t, 1 \leq j \leq l, 0<i_{1} \leq i_{2} \leq \ldots \leq$ 
$i_{l-1}<i_{l}$, e existe pelo menos um idempotente $\left(\frac{1+d}{2}\right) e_{i}$ e $\left(\frac{1-d}{2}\right) e_{j}$ como geradores dos ideais somandos de $C$, então $w(C)=4\left|G_{i_{l}}\right|$.

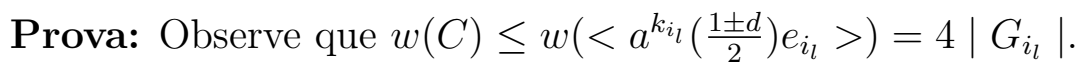

Temos $C \subset<a^{k} \widehat{G}_{i_{l}}>$, onde $k=\min \left\{k_{i_{1}}, \ldots, k_{i_{l}}\right\}$. Sejam $\Gamma=\left\{\tau_{1}, \ldots, \tau_{n}\right\}$ um transversal de $G_{i_{l}}$ em $G$ e $\alpha \in C$. Podemos escrever $\alpha=\left(x_{1} \tau_{1}+\ldots+x_{n} \tau_{n}+x_{1}^{\prime} d \tau_{1}+\ldots+x_{n}^{\prime} d \tau_{n}\right) a^{k} \widehat{G}_{i_{l}}$, onde $x_{j}$ e $x_{j}^{\prime} \in R$. Como $\alpha \in C$, existem $\beta_{1}, \ldots, \beta_{l} \in R A$ tais que

$$
\alpha=\beta_{1} a^{k_{i_{1}}}\left(\frac{1 \pm d}{2}\right) e_{i_{1}}+\ldots+\beta_{l} a^{k_{i_{l}}}\left(\frac{1 \pm d}{2}\right) e_{i_{l}}
$$

Suponhamos que exista apenas um coeficiente $y$ de $\alpha$ tal que $y a^{k} \neq 0$. Então $\alpha=\left(y d^{\zeta_{\tau}} \tau\right) a^{k} \widehat{G}_{i_{l}}=\beta_{1} a^{k_{i_{1}}}\left(\frac{1 \pm d}{2}\right) e_{i_{1}}+\ldots+\beta_{l} a^{k_{i_{l}}}\left(\frac{1 \pm d}{2}\right) e_{i_{l}}$, onde $\tau \in \Gamma$.

Multiplicando a igualdade acima por $\widehat{G}_{i_{1}-1}$, temos $\left(y d^{\zeta_{\tau}} \tau\right) a^{k} \widehat{G}_{i_{1}-1}=0$. Assim, $y a^{k}=0$, o que contradiz nossa hipótese.

Suponhamos agora que existam apenas dois coeficientes $y_{1}, y_{2}$ de $\alpha$ tais que $y_{1} a^{k} \neq 0$ e $y_{2} a^{k} \neq 0$. Assim, podemos escrever $\alpha$ como

$$
\alpha=\left(y_{1} d^{\zeta_{\tau}} \tau+y_{2} d^{\zeta_{\tau^{\prime}}} \tau^{\prime}\right) a^{k} \widehat{G}_{i_{l}}=\beta_{1} a^{k_{i_{1}}}\left(\frac{1 \pm d}{2}\right) e_{i_{1}}+\ldots+\beta_{l} a^{k_{i_{l}}}\left(\frac{1 \pm d}{2}\right) e_{i_{l}}
$$

onde $\zeta_{\tau}, \zeta_{\tau^{\prime}}=0$ ou 1 e $\tau, \tau^{\prime} \in \Gamma$. Suponhamos que o idempotente gerador do último ideal somando de $C$ seja $\left(\frac{1+d}{2}\right) e_{i_{l}}$, caso contrário, refaça o mesmo que for feito abaixo para $\left(\frac{1-d}{2}\right) e_{i_{l}}$.

Multiplicando a igualdade (4.5) por $\left(\frac{1-d}{2}\right)$, temos

$$
\left(y_{1} d^{\zeta_{\tau}} \tau+y_{2} d^{\zeta_{\tau^{\prime}}} \tau^{\prime}\right) a^{k}\left(\frac{1-d}{2}\right) \widehat{G}_{i_{l}}=\beta_{1} a^{k_{j_{1}}}\left(\frac{1-d}{2}\right) e_{j_{1}}+\ldots+\beta_{l} a^{k_{j_{s}}}\left(\frac{1-d}{2}\right) e_{j_{s}},
$$

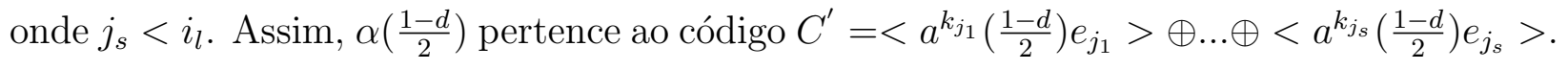
Mas $w\left(\alpha\left(\frac{1-d}{2}\right)\right)=4\left|G_{i_{l}}\right|$ e $w\left(C^{\prime}\right)=4\left|G_{j_{s}}\right|$. Como $\left|G_{i_{l}}\right|<\left|G_{j_{s}}\right|$, temos uma contradição.

Suponhamos agora que existam apenas três coeficientes $y_{1}, y_{2}$ e $y_{3}$ de $\alpha$ tais que $y_{1} a^{k} \neq 0$, 
$y_{2} a^{k} \neq 0$ e $y_{3} a^{k} \neq 0$. Assim, podemos escrever $\alpha$ como

$$
\alpha=\left(y_{1} d^{\zeta_{\tau}} \tau+y_{2} d^{\zeta_{\tau^{\prime}}} \tau^{\prime}+y_{3} d^{\zeta_{\tau^{\prime \prime}}} \tau^{\prime \prime}\right) a^{k} \widehat{G}_{i_{l}}=\beta_{1} a^{k_{i_{1}}}\left(\frac{1 \pm d}{2}\right) e_{i_{1}}+\ldots+\beta_{l} a^{k_{i_{l}}}\left(\frac{1 \pm d}{2}\right) e_{i_{l}}
$$

onde $\zeta_{\tau}, \zeta_{\tau^{\prime}}, \zeta_{\tau^{\prime \prime}}=1$ ou 0 e $\tau, \tau^{\prime}, \tau^{\prime \prime} \in \Gamma$.

Suponhamos que o idempotente gerador do ultimo ideal somando de $C$ seja $\left(\frac{1+d}{2}\right) e_{i_{l}}$, caso contrário, refaça o mesmo que for feito abaixo para $\left(\frac{1-d}{2}\right) e_{i_{l}}$.

Multiplicando a igualdade (4.6) por $\left(\frac{1-d}{2}\right)$, temos

$$
\left(y_{1} d^{\zeta_{\tau}} \tau+y_{2} d^{\zeta_{\tau^{\prime}}} \tau^{\prime}+y_{3} d_{\tau^{\prime \prime}}^{\zeta^{\prime \prime}}\right) a^{k}\left(\frac{1-d}{2}\right) \widehat{G}_{i_{l}}=\beta_{1} a^{k_{j_{1}}}\left(\frac{1-d}{2}\right) e_{j_{1}}+\ldots+\beta_{l} a^{k_{j_{s}}}\left(\frac{1-d}{2}\right) e_{j_{s}}
$$

onde $j_{s}<i_{l}$. Assim, $\alpha\left(\frac{1-d}{2}\right)$ pertence ao código $C^{\prime}=<a^{k_{j_{1}}}\left(\frac{1-d}{2}\right) e_{j_{1}}>\oplus \ldots \oplus<a^{k_{j_{s}}}\left(\frac{1-d}{2}\right) e_{j_{s}}>$. $\operatorname{Mas} w\left(\alpha\left(\frac{1-d}{2}\right)\right)=6\left|G_{i_{l}}\right|$ e $w\left(C^{\prime}\right)=4\left|G_{j_{s}}\right|$. Como $4\left|G_{j_{s}}\right|=4 p^{n-i_{l}}\left(p^{i_{l}-j_{s}}\right)$ e $j_{s}<i_{l}$, temos $\left(p^{i_{l}-j_{s}}\right)>3, \operatorname{logo} 6\left|G_{i_{l}}\right|<4\left|G_{j_{s}}\right|$, o que é uma contradição.

Assim, $w(C) \geq 4\left|G_{i_{l}}\right|$ e, portando, $w(C)=4\left|G_{i_{l}}\right|$

Teorema 4.1.11 Considere $R$ e $G$ nas condições da Hipótese B, com $p>3$. Se o código $C$ é da forma $C=<a^{k_{00}}\left(\frac{1+d}{2}\right) e_{0}>\oplus<a^{k_{01}}\left(\frac{1-d}{2}\right) e_{0}>\oplus<a^{k_{10}}\left(\frac{1+d}{2}\right) e_{1}>\oplus<a^{k_{11}}\left(\frac{1-d}{2}\right) e_{1}>$

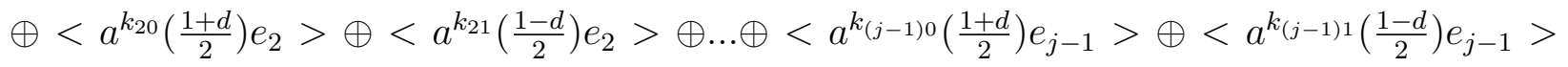
$\oplus<a^{k_{j}}\left(\frac{1 \pm d}{2}\right) e_{j}>$, onde $0<j \leq n, 0 \leq k_{i 0}, k_{i 1} \leq t$, para $0<i \leq j, k_{j}<t$ e $k_{00}$ ou $k_{01}<t$, e existem pelo menos dois ideais da forma $<a^{k_{r}}\left(\frac{1+d}{2}\right) e_{r}>e<a^{k_{s}}\left(\frac{1-d}{2}\right) e_{s}>$ não nulos, então $w(C)=4\left|G_{j}\right|$.

Prova: Temos $w(C) \leq 4 \quad\left|\quad G_{j} \quad\right| \quad$ e $\quad C \quad \subset \quad a^{k} \widehat{G}_{j}$, onde $k=\min \left\{k_{0}, k_{1}, k_{20}, k_{21}, \ldots, k_{(j-1) 0}, k_{(j-1) 1}, k_{j}\right\} . \quad$ Sejam $\Gamma=\left\{\tau_{1}, \ldots, \tau_{n}\right\}$ um transversal de 
$G_{j}$ em $G$ e $\alpha \in C$. Então podemos escrever

$$
\begin{aligned}
\alpha= & \left(x_{1} \tau_{1}+\ldots+x_{n} \tau_{n}+x_{1}^{\prime} d \tau_{1}+\ldots+x_{n}^{\prime} d \tau_{n}\right) a^{k} \widehat{G}_{j} \\
= & \beta_{00} a^{k_{00}}\left(\frac{1+d}{2}\right) e_{0}+\beta_{01} a^{k_{01}}\left(\frac{1-d}{2}\right) e_{0}+\beta_{10} a^{k_{10}}\left(\frac{1+d}{2}\right) e_{1}+\beta_{11} a^{k_{11}}\left(\frac{1-d}{2}\right) e_{1}+ \\
& +\beta_{20} a^{k_{20}}\left(\frac{1+d}{2}\right) e_{2}+\beta_{21} a^{k_{21}}\left(\frac{1-d}{2}\right) e_{2}+\ldots+\beta_{j} a^{k_{j}}\left(\frac{1 \pm d}{2}\right) e_{j},
\end{aligned}
$$

onde $\beta_{00}, \beta_{01}, \beta_{10}, \beta_{11}, \ldots, \beta_{j} \in R A$.

Vamos supor que o último ideal é da forma $\left\langle a^{k_{j}}\left(\frac{1-d}{2}\right) e_{j}\right\rangle$, sendo o cálculo análogo para $<a^{k_{j}}\left(\frac{1+d}{2}\right) e_{j}>$

Suponhamos que exista apenas um coeficiente $y$ de $\alpha$ tal que $y a^{k} \neq 0$. Então $\alpha=\left(y d^{\zeta_{\tau}} \tau\right) a^{k} \widehat{G}_{j}=\beta_{00} a^{k_{00}}\left(\frac{1+d}{2}\right) e_{0}+\beta_{01} a^{k_{01}}\left(\frac{1-d}{2}\right) e_{0}+\beta_{10} a^{k_{10}}\left(\frac{1+d}{2}\right) e_{1}+\beta_{11} a^{k_{11}}\left(\frac{1-d}{2}\right) e_{1}+$ $\beta_{20} a^{k_{20}}\left(\frac{1+d}{2}\right) e_{2}+\beta_{21} a^{k_{21}}\left(\frac{1-d}{2}\right) e_{2}+\ldots+\beta_{j} a^{k_{j}}\left(\frac{1-d}{2}\right) e_{j}$, onde $\tau \in \Gamma$.

Multiplicando a igualdade acima por $\left(\frac{1+d}{2}\right)$, temos $\left(y d^{\zeta_{\tau}} \tau\right) a^{k}\left(\frac{1+d}{2}\right) \widehat{G}_{j}=\beta_{00} a^{k_{00}}\left(\frac{1+d}{2}\right) e_{0}+$ $\beta_{10} a^{k_{10}}\left(\frac{1+d}{2}\right) e_{1}+\ldots+\beta_{r 0} a^{k_{r 0}}\left(\frac{1+d}{2}\right) e_{r}$, onde $r<j$.

Logo a palavra $\left(y d^{\zeta_{\tau}} \tau\right) a^{k}\left(\frac{1+d}{2}\right) \widehat{G}_{j}$ pertence ao código

$$
C^{\prime}=<a^{k_{00}}\left(\frac{1+d}{2}\right) e_{0}>\oplus<a^{k_{10}}\left(\frac{1+d}{2}\right) e_{1}>\oplus \ldots \oplus<a^{k_{r 0}}\left(\frac{1+d}{2}\right) e_{r}>
$$

Temos $w\left(\left(y d^{\zeta_{\tau}} \tau\right) a^{k}\left(\frac{1+d}{2}\right) \widehat{G}_{j}\right)=2\left|G_{j}\right|$ e $w\left(C^{\prime}\right) \in\left\{2\left|G_{r}\right|, 4\left|G_{r}\right|\right.$ ou $\left.|A|\right\}$. Como $p>3$, temos $2\left|G_{j}\right|<2\left|G_{r}\right|<4\left|G_{r}\right|<|A|$. Assim, a palavra $\left(y d^{\zeta_{\tau}} \tau\right) a^{k}\left(\frac{1+d}{2}\right) \widehat{G}_{j}=0$, $\log 0 y a^{k}=0$, o que contradiz nossa hipótese inicial.

Suponhamos agora que existam apenas dois coeficientes $y_{1}, y_{2}$ de $\alpha$ tais que $y_{1} a^{k} \neq 0$ e $y_{2} a^{k} \neq 0$. Então $\alpha=\left(y_{1} d^{\zeta_{\tau}} \tau+y_{2} d^{\zeta_{\tau^{\prime}}} \tau^{\prime}\right) a^{k} \widehat{G}_{j}=\beta_{00} a^{k_{00}}\left(\frac{1+d}{2}\right) e_{0}+\beta_{01} a^{k_{01}}\left(\frac{1-d}{2}\right) e_{0}+$ $\beta_{10} a^{k_{10}}\left(\frac{1+d}{2}\right) e_{1}+\beta_{11} a^{k_{11}}\left(\frac{1-d}{2}\right) e_{1}+\beta_{20} a^{k_{20}}\left(\frac{1+d}{2}\right) e_{2}+\beta_{21} a^{k_{21}}\left(\frac{1-d}{2}\right) e_{2}+\ldots+\beta_{j} a^{k_{j}}\left(\frac{1-d}{2}\right) e_{j}$, onde $\tau$ e $\tau^{\prime} \in \Gamma$ e $\zeta_{\tau}$ e $\zeta_{\tau^{\prime}}=0$ ou 1 .

Multiplicando a igualdade acima por $\left(\frac{1+d}{2}\right)$, temos

$$
\left(y_{1} d^{\zeta_{\tau}} \tau+y_{2} d^{\zeta_{\tau^{\prime}}} \tau^{\prime}\right) a^{k}\left(\frac{1+d}{2}\right) \widehat{G}_{j}=\beta_{00} a^{k_{00}}\left(\frac{1+d}{2}\right) e_{0}+\beta_{10} a^{k_{10}}\left(\frac{1+d}{2}\right) e_{1}+\ldots+\beta_{j_{l} 0} a^{k_{j_{l} 0}}\left(\frac{1+d}{2}\right) e_{j_{l}}
$$


onde $j_{l}<j . \quad$ Logo a palavra $\left(y_{1} d^{\zeta_{\tau}} \tau+y_{2} d_{\tau^{\prime}}^{\zeta^{\prime}}\right) a^{k}\left(\frac{1+d}{2}\right) \widehat{G}_{j}$ pertence ao código $C^{\prime}=<a^{k_{00}}\left(\frac{1+d}{2}\right) e_{0}>\oplus<a^{k_{10}}\left(\frac{1+d}{2}\right) e_{1}>\oplus \ldots \oplus<a^{k_{j_{l} 0}}\left(\frac{1+d}{2}\right) e_{j_{l}}>$. Assim,

$$
w\left(\left(y_{1} d^{\zeta_{\tau}} \tau+y_{2} d^{\zeta_{\tau^{\prime}}} \tau^{\prime}\right) a^{k}\left(\frac{1+d}{2}\right) \widehat{G}_{j}\right)=4\left|G_{j}\right|
$$

e

$$
w\left(C^{\prime}\right) \in\left\{2\left|G_{j_{l}}\right|, 4\left|G_{j_{l}}\right| \text { ou }|A|\right\}
$$

Como $p>3$, temos $4\left|G_{j}\right|<2\left|G_{j_{l}}\right|<4\left|G_{j_{l}}\right|<|A|$. Assim,

$$
\left(y_{1} d^{\zeta_{\tau}} \tau+y_{2} d_{\tau^{\prime}}^{\zeta^{\prime}}\right) a^{k}\left(\frac{1+d}{2}\right) \widehat{G}_{j}=0
$$

Desenvolvendo a igualdade acima, temos $\frac{1}{2}\left(\left(y_{1} \tau+y_{2} \tau^{\prime}\right) a^{k} \widehat{G}_{j}+\left(y_{1} \tau+y_{2} \tau^{\prime}\right) a^{k} d \widehat{G}_{j}\right)=0$. Como $\left(y_{1} \tau+y_{2} \tau^{\prime}\right) a^{k} \widehat{G}_{j}$ e $\left(y_{1} \tau+y_{2} \tau^{\prime}\right) a^{k} d \widehat{G}_{j}$ têm suportes disjuntos, temos

$$
\left(y_{1} \tau+y_{2} \tau^{\prime}\right) a^{k} \widehat{G}_{j}=0
$$

assim $y_{1} a^{k}=y_{2} a^{k}=0$, o que contradiz nossa hipótese inicial.

Suponhamos agora que existam apenas três coeficientes $y_{1}, y_{2}$ e $y_{3}$ de $\alpha$ tais que $y_{1} a^{k} \neq 0$, $y_{2} a^{k} \neq 0$ e $y_{3} a^{k} \neq 0$. Então $\alpha=\left(y_{1} d^{\zeta_{\tau}} \tau+y_{2} d^{\zeta_{\tau^{\prime}}} \tau^{\prime}+y_{3} d^{\zeta_{\tau^{\prime \prime}}} \tau^{\prime \prime}\right) a^{k} \widehat{G}_{j}=\beta_{00} a^{k_{00}}\left(\frac{1+d}{2}\right) e_{0}+$ $\beta_{01} a^{k_{01}}\left(\frac{1-d}{2}\right) e_{0}+\beta_{10} a^{k_{10}}\left(\frac{1+d}{2}\right) e_{1}+\beta_{11} a^{k_{11}}\left(\frac{1-d}{2}\right) e_{1}+\beta_{20} a^{k_{20}}\left(\frac{1+d}{2}\right) e_{2}+\beta_{21} a^{k_{21}}\left(\frac{1-d}{2}\right) e_{2}+\ldots+$ $\beta_{j} a^{k_{j}}\left(\frac{1-d}{2}\right) e_{j}$, onde $\tau, \tau^{\prime}$ e $\tau^{\prime \prime} \in \Gamma$.

Multiplicando a igualdade acima por $\left(\frac{1+d}{2}\right)$, temos

$$
\begin{aligned}
\left(y_{1} d^{\zeta_{\tau}} \tau+y_{2} d^{\zeta_{\tau^{\prime}}} \tau^{\prime}+y_{3} d^{\zeta_{\tau^{\prime \prime}}} \tau^{\prime \prime}\right) a^{k}\left(\frac{1+d}{2}\right) \widehat{G}_{j}= & \beta_{00} a^{k_{00}}\left(\frac{1+d}{2}\right) e_{0}+\beta_{10} a^{k_{10}}\left(\frac{1+d}{2}\right) e_{1}+\ldots+ \\
& +\beta_{j_{l} 0} a^{k_{j_{l} 0}\left(\frac{1+d}{2}\right) e_{j_{l}}}
\end{aligned}
$$

onde $j_{l}<j$.

Logo a palavra $\left(y_{1} d^{\zeta \tau} \tau+y_{2} d^{\zeta_{\tau^{\prime}}} \tau^{\prime}+y_{3} d_{\tau^{\prime \prime}} \tau^{\prime \prime}\right) a^{k}\left(\frac{1+d}{2}\right) \widehat{G}_{j}$ pertence ao código 
$C^{\prime}=<a^{k_{00}}\left(\frac{1+d}{2}\right) e_{0}>\oplus<a^{k_{10}}\left(\frac{1+d}{2}\right) e_{1}>+\ldots+<a^{k_{j_{l} 0}}\left(\frac{1+d}{2}\right) e_{j_{l}}>$. Assim,

$$
w\left(\left(y_{1} d^{\zeta_{\tau}} \tau+y_{2} d_{\tau^{\prime}} \tau^{\prime}+y_{3} d^{\zeta_{\tau^{\prime \prime}}} \tau^{\prime \prime}\right) a^{k}\left(\frac{1+d}{2}\right) \widehat{G}_{j}\right)=6\left|G_{j}\right|
$$

e

$$
w\left(C^{\prime}\right) \in\left\{2\left|G_{j_{l}}\right|, 4\left|G_{j_{l}}\right| \text { ou }|A|\right\}
$$

Como $p>3$, temos $6\left|G_{j}\right|<2\left|G_{j_{l}}\right|<4\left|G_{j_{l}}\right|<|A|$. Assim,

$$
\left(y_{1} d^{\zeta_{\tau}} \tau+y_{2} d_{\tau^{\prime}}^{\zeta^{\prime}}+y_{3} d_{\tau^{\prime \prime}}^{\zeta^{\prime \prime}}\right) a^{k}\left(\frac{1+d}{2}\right) \widehat{G}_{j}=0
$$

Desenvolvendo a igualdade acima, temos $\frac{1}{2}\left(\left(y_{1} \tau+y_{2} \tau^{\prime}+y_{3} \tau^{\prime \prime}\right) a^{k} \widehat{G}_{j}+\left(y_{1} \tau+y_{2} \tau^{\prime}+\right.\right.$ $\left.\left.y_{3} \tau^{\prime \prime}\right) a^{k} d \widehat{G}_{j}\right)=0$. Como $\left(y_{1} \tau+y_{2} \tau^{\prime}+y_{3} \tau^{\prime \prime}\right) a^{k} \widehat{G}_{j}$ e $\left(y_{1} \tau+y_{2} \tau^{\prime}+y_{3} \tau^{\prime \prime}\right) a^{k} d \widehat{G}_{j}$ têm suportes disjuntos, temos

$$
\left(y_{1} \tau+y_{2} \tau^{\prime}+y_{3} \tau^{\prime \prime}\right) a^{k} \widehat{G}_{j}=0
$$

assim $y_{1} a^{k}=y_{2} a^{k}=y_{3} a^{k} 0$, o que contradiz nossa hipótese inicial.

Portanto $w(C) \geq 4\left|G_{j}\right|$ e, como tínhamos $w(C) \leq 4\left|G_{j}\right|$, obtemos $w(C)=4\left|G_{j}\right|$.

Nos teoremas anteriores, sempre consideramos o último ideal somando dos códigos como sendo ou $a^{k_{1}}\left(\frac{1+d}{2}\right) e_{j}$ ou $a^{k_{2}}\left(\frac{1-d}{2}\right) e_{j}$. Agora iremos considerar o caso em que existem ambos ideais, ou seja, os geradores do dois últimos ideais dos códigos em questão são $a^{k_{j 0}}\left(\frac{1+d}{2}\right) e_{j}$ e $a^{k_{j 1}}\left(\frac{1-d}{2}\right) e_{j}$, onde $k_{j 0}, k_{j 1}<t$.

Teorema 4.1.12 Considere $R$ e $G$ nas condições da Hipótese B. Se o código $C$ é da forma $C=<a^{k_{i_{1}}}\left(\frac{1 \pm d}{2}\right) e_{i_{1}}>\oplus<a^{k_{i_{2}}\left(\frac{1 \pm d}{2}\right)} e_{i_{2}}>\oplus \ldots \oplus<a^{k_{i_{1}}}\left(\frac{1+d}{2}\right) e_{i_{l}}>\oplus<a^{k_{i_{2}}}\left(\frac{1-d}{2}\right) e_{i_{l}}>$, onde $0 \leq k_{i_{j}}<t, 1 \leq j \leq l, 0<i_{1} \leq i_{2} \leq \ldots \leq i_{l-1}<i_{l}$, então $w(C)=2\left|G_{i_{l}}\right|$.

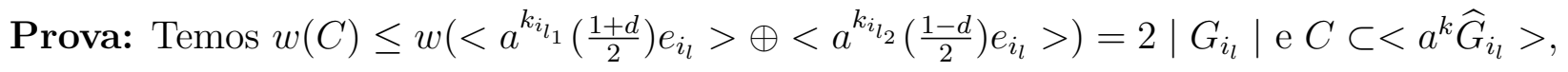
onde $k=\min \left\{k_{i_{1}}, k_{i_{2}}, \ldots, k_{i_{1}}, k_{i_{l_{2}}}\right\}$. Sejam $\Gamma=\left\{\tau_{1}, \ldots, \tau_{n}\right\}$ um transversal de $G_{i_{l}}$ em $G$ e 
$\alpha \in C$.

Podemos escrever $\alpha=\left(x_{1} \tau_{1}+\ldots+x_{n} \tau_{n}+x_{1}^{\prime} d \tau_{1}+\ldots+x_{n}^{\prime} d \tau_{n}\right) a^{k} \widehat{G}_{j_{l}}$. Como

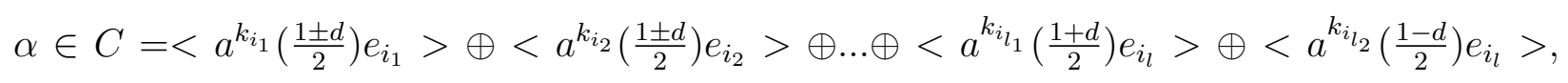
existem $\beta_{1}, \ldots, \beta_{l_{1}}, \beta_{l_{2}} \in R A$ tais que

$$
\alpha=\beta_{1} a^{k_{i_{1}}}\left(\frac{1 \pm d}{2}\right) e_{i_{1}}+\ldots+\beta_{l_{1}} a^{k_{i_{1}}}\left(\frac{1+d}{2}\right) e_{i_{l}}+\beta_{l_{2}} a^{k_{i_{2}}}\left(\frac{1-d}{2}\right) e_{i_{l}}
$$

Vamos supor que exista apenas um coeficiente $y$ de $\alpha$, tal que $y a^{k} \neq 0$. Então

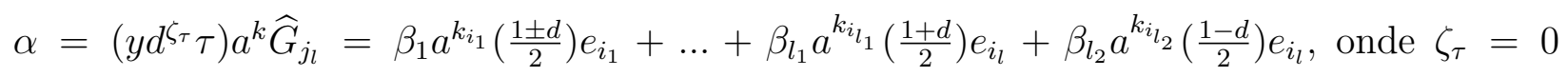
ou 1 e $\tau \in \Gamma$.

Multiplicando a igualdade acima por $\widehat{G}_{i_{1}-1}$, temos $\left(y d^{\zeta_{\tau}} \tau\right) a^{k} \widehat{G}_{i_{1}-1}=0$. Logo $y a^{k}=0$, o que contradiz nossa hipótese, assim $w(C) \geq 2\left|G_{i_{l}}\right|$. Portanto, $w(C)=2\left|G_{i_{l}}\right|$

Teorema 4.1.13 Considere $R$ e G nas condições da Hipótese B. Se o código $C$ é da forma $C=<a^{k_{00}}\left(\frac{1+d}{2}\right) e_{0}>\oplus<a^{k_{01}}\left(\frac{1-d}{2}\right) e_{0}>\oplus<a^{k_{10}}\left(\frac{1+d}{2}\right) e_{1}>\oplus<a^{k_{11}}\left(\frac{1-d}{2}\right) e_{1}>\oplus$

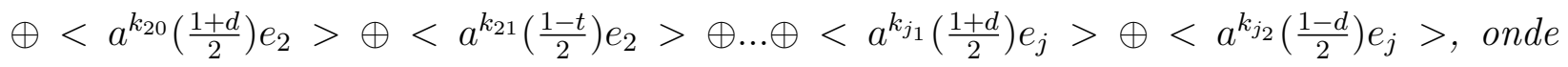
$0<j \leq n, k_{00}$ ou $k_{01}<t$, e $k_{j_{1}}$ e $k_{j_{2}}<t$ então $w(C)=2\left|G_{j}\right|$ ou $\left|G_{j}\right|$.

Prova: Dividiremos a demonstração em duas partes. Vamos supor inicialmente que exista $r$ tal que $k_{r 0}$ ou $k_{r 1}$ seja igual a $t$ e provaremos que $w(C)=2\left|G_{j}\right|$ e depois consideraremos o caso em que $k_{i 0}, k_{i 1}<t$, para $0 \leq i \leq j-1$ e provaremos que $w(C)=\left|G_{j}\right|$.

Observe que $w(C) \leq w\left(<a^{k_{j_{1}}}\left(\frac{1+d}{2}\right) e_{j}>\oplus<a^{k_{j_{2}}}\left(\frac{1-d}{2}\right) e_{j}>\right)=2\left|G_{j}\right|$. Falta provar que $w(C) \geq 2\left|G_{j}\right|$.

Temos $C \subset<a^{k} \widehat{G}_{j}>$, onde $k=\min \left\{k_{00}, k_{01}, k_{10}, k_{11}, \ldots, k_{j}\right\}$. Sejam $\Gamma=\left\{\tau_{1}, \ldots, \tau_{n}\right\}$ um transversal de $G_{j}$ em $G$ e $\alpha \in C$.

Podemos escrever $\alpha=\left(x_{1} \tau_{1}+\ldots+x_{n} \tau_{n}+x_{1}^{\prime} d \tau_{1}+\ldots+x_{n}^{\prime} d \tau_{n}\right) a^{k} \widehat{G}_{j}$.

Como $\alpha \in C=<a^{k_{00}}\left(\frac{1+d}{2}\right) e_{0}>\oplus<a^{k_{01}}\left(\frac{1-d}{2}\right) e_{0}>\oplus<a^{k_{10}}\left(\frac{1+d}{2}\right) e_{1}>\oplus<a^{k_{11}}\left(\frac{1-d}{2}\right) e_{1}>$

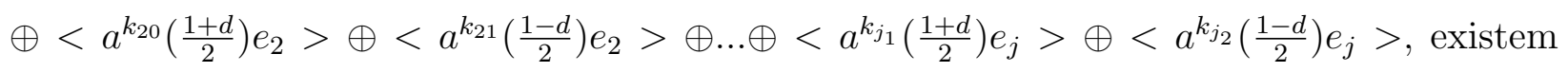


$\beta_{00}, \beta_{01}, \ldots, \beta_{j_{1}}, \beta_{j_{2}} \in R A$, tal que $\alpha=\beta_{00} a^{k_{00}}\left(\frac{1+d}{2}\right) e_{0}+\beta_{01} a^{k_{01}}\left(\frac{1-d}{2}\right) e_{0}+\ldots+\beta_{j_{1}}\left(\frac{1+d}{2}\right) a^{k_{j_{1}}} e_{j}+$ $\beta_{j_{2}}\left(\frac{1-d}{2}\right) a^{k_{j_{2}}} e_{j}$.

Vamos supor que exista apenas um coeficiente $y$ de $\alpha$ tal que $y a^{k} \neq 0$. Então $\alpha=\left(y d^{\zeta \tau} \tau\right) a^{k} \widehat{G}_{j}=\beta_{00} a^{k_{00}}\left(\frac{1+d}{2}\right) e_{0}+\beta_{01} a^{k_{01}}\left(\frac{1-d}{2}\right) e_{0}+\ldots+\beta_{j_{1}}\left(\frac{1+d}{2}\right) a^{k_{j_{1}}} e_{j}+\beta_{j_{2}}\left(\frac{1-d}{2}\right) a^{k_{j_{2}}} e_{j}$,

onde $\tau \in \Gamma$. Agora vamos supor, sem perda de generalidade, que $i$ é o menor índice tal que $k_{i 0}=t$ e, assim, $a^{k_{i 0}}=0$. Multiplicando a igualdade acima por $\frac{1+d}{2} \widehat{G}_{i}$, temos:

$$
\left(y d^{\zeta_{\tau}} \tau\right) a^{k}\left(\frac{1+d}{2}\right) \widehat{G}_{i}=\beta_{00} a^{k_{00}}\left(\frac{1+d}{2}\right) e_{0}+\beta_{10} a^{k_{10}}\left(\frac{1+d}{2}\right) e_{1}+\ldots+\beta_{i-1} a^{k_{i-10}}\left(\frac{1+d}{2}\right) e_{i-1 .} .
$$

Se $i=0$, então $\left(y d^{\zeta_{\tau}} \tau\right) a^{k}\left(\frac{1+d}{2}\right) \widehat{G}_{i}=0$ e assim $y a^{k}=0$, o que contradiz nossa hipótese.

Se $i>0$, a palavra $\left(y d^{\zeta_{\tau}} \tau\right) a^{k}\left(\frac{1+d}{2}\right) \widehat{G}_{i}$ pertence ao código $C^{\prime}=<a^{k_{00}}\left(\frac{1+d}{2}\right) e_{0}>\oplus$ $\oplus<a^{k_{10}}\left(\frac{1+d}{2}\right) e_{1}>\oplus \ldots \oplus<a^{k_{i-10}}\left(\frac{1+d}{2}\right) e_{i-1}>$. O menor peso possível para $C^{\prime}$ é $2\left|G_{i-1}\right|$, mas o peso de $\left(y d^{\zeta_{\tau}} \tau\right) a^{k}\left(\frac{1+d}{2}\right) \widehat{G}_{i}$ é $4\left|G_{i}\right|$ que é menor que $2\left|G_{i-1}\right|$, pois $p>2$. Assim, temos uma contradição. Portanto, $w(C) \geq 2\left|G_{j}\right|$.

Suponhamos agora $k_{i 0}, k_{i 1}<t$, para $0 \leq i \leq j-1$. Temos $C \subset<\widehat{G}_{j}>$. Portanto, $w(C) \geq w\left(<\widehat{G}_{j}>\right)=\left|G_{j}\right|$.

Agora, seja $k=\max \left\{k_{00}, k_{01}, \ldots, k_{j-10}, k_{j-11}, k_{j}\right\}$. Assim,

$$
\begin{aligned}
<a^{k} \widehat{G}_{j}>= & <a^{k}\left(\left(\widehat{G}_{j}-\widehat{G}_{j-1}\right)+\left(\widehat{G}_{j-1}-\widehat{G}_{j-2}\right)+\ldots+\left(\widehat{G}_{1}-\widehat{G}_{0}\right)+\widehat{G}_{0}\right)> \\
= & <a^{k}\left(e_{j}+e_{j-1}+\ldots+e_{1}+e_{0}\right)> \\
= & \left.<a^{k}\left(\frac{1+d}{2}\right) e_{0}+a^{k}\left(\frac{1-d}{2}\right) e_{0}+\ldots+a^{k}\left(\frac{1+d}{2}\right) e_{j}+a^{k}\left(\frac{1-d}{2}\right) e_{j}\right)> \\
\subset & <a^{k_{00}}\left(\frac{1+d}{2}\right) e_{0}>\oplus<a^{k_{01}}\left(\frac{1-d}{2}\right) e_{0}>\oplus<a^{k_{10}}\left(\frac{1+d}{2}\right) e_{1}>\oplus<a^{k_{11}}\left(\frac{1-d}{2}\right) e_{1}>\oplus \ldots \oplus \\
& \oplus<a^{k_{j_{1}}}\left(\frac{1+d}{2}\right) e_{j}>\oplus<a^{k_{j_{2}}}\left(\frac{1-d}{2}\right) e_{j}> \\
= & C .
\end{aligned}
$$

$\operatorname{Logo}\left|G_{j}\right|=w\left(<a^{k} \widehat{G}_{j}>\right) \geq w(C)$. Portanto, $w(C)=\left|G_{j}\right|$. 
Agora estamos interessados em calcular o número de palavras de um dado código. Iniciaremos então o cálculo para os ideais $\left\langle a^{k}\left(\frac{1+d}{2}\right) e_{i}\right\rangle$.

Teorema 4.1.14 Se o código $C$ é da forma $C=<a^{k}\left(\frac{1+d}{2}\right) e_{i}>$, então o número de palavras de $C$ é $\left|<a^{k}\left(\frac{1+d}{2}\right) e_{i}>\right|=|\bar{R}|^{(t-k)\left(p^{i}-p^{i-1}\right)}$, se $i>0$ e $\left|<a^{k}\left(\frac{1+d}{2}\right) e_{0}>\right|=|\bar{R}|^{t-k}$.

Prova: Observe que se $i>0$, então

$$
a^{k}\left(\frac{1+d}{2}\right) e_{i}=a^{k}\left(\frac{1+d}{2}\right)\left(\widehat{G}_{i}-\widehat{G}_{i-1}\right) .
$$

Logo

$$
a^{k}\left(\frac{1+d}{2}\right) \widehat{G}_{i}=a^{k}\left(\frac{1+d}{2}\right) e_{i}+a^{k}\left(\frac{1+d}{2}\right) \widehat{G}_{i-1}
$$

Como

$$
a^{k}\left(\frac{1+d}{2}\right) e_{i} \cdot a^{k}\left(\frac{1+d}{2}\right) \widehat{G}_{i-1}=0
$$

temos que

$$
R A a^{k}\left(\frac{1+d}{2}\right) \widehat{G}_{i}=R A a^{k}\left(\frac{1+d}{2}\right) e_{i} \oplus R A a^{k}\left(\frac{1+d}{2}\right) \widehat{G}_{i-1}
$$

Assim,

$$
\left|R A a^{k}\left(\frac{1+d}{2}\right) e_{i}\right|=\frac{\left|R A a^{k}\left(\frac{1+d}{2}\right) \widehat{G}_{i}\right|}{\left|R A a^{k}\left(\frac{1+d}{2}\right) \widehat{G}_{i-1}\right|} .
$$

Agora iremos calcular $\left|R A a^{k}\left(\frac{1+d}{2}\right) \widehat{G}_{i}\right|$.

Considere

$$
\begin{array}{cc}
\psi: R A & \rightarrow R A a^{k} . \\
\alpha & \mapsto \alpha a^{k}
\end{array}
$$

Claramente $\psi$ é um epimorfismo de grupos aditivos. O núcleo de $\psi$ é dado por

$$
\operatorname{ker}(\psi)=\left\{\alpha \in R A ; \alpha a^{k}=0\right\}
$$


Pelo Lema 2.1.6, temos que $\alpha a^{k}=0$ se, e somente se, $\alpha \in<a^{t-k}>A$. Portanto,

$$
R A a^{k} \simeq \frac{R A}{<a^{t-k}>A}
$$

Como $\frac{R A}{<a^{t-k}>A} \simeq\left(\frac{R}{<a^{t-k}>}\right) A$, temos

$$
\left|R A a^{k}\left(\frac{1+d}{2}\right) \widehat{G}_{i}\right|=\left|\left(\frac{R}{<a^{t-k}>}\right) A\left(\frac{1+d}{2}\right) \widehat{G}_{i}\right|
$$

Agora considere $R^{\prime}=\left(\frac{R}{<a^{t-k}>}\right)$ e observe que

$$
R^{\prime} A\left(\frac{1+d}{2}\right) \widehat{G}_{i}=\left\{\left(\sum_{g \in G} x_{g} g+\sum_{h \in G} y_{h} d h\right)\left(\frac{1+d}{2}\right) \widehat{G}_{i} ; x_{g}, y_{h} \in R^{\prime}, g \in G, d \in B\right\}
$$

Como $d \cdot \frac{1+d}{2}=\frac{1+d}{2}$, podemos escrever $R^{\prime} A\left(\frac{1+d}{2}\right) \widehat{G}_{i}$ como

$$
R^{\prime} A\left(\frac{1+d}{2}\right) \widehat{G}_{i}=\left\{\left(\sum_{g \in G} x_{g} g\right)\left(\frac{1+d}{2}\right) \widehat{G}_{i} ; x_{g} \in R^{\prime}, g \in G, d \in B\right\}
$$

Considere

$$
\begin{aligned}
\Phi: R^{\prime} A\left(\frac{1+d}{2}\right) \widehat{G}_{i} & \rightarrow R^{\prime} G \widehat{G}_{i} . \\
\left(\sum_{g \in G} x_{g} g\right)\left(\frac{1+d}{2}\right) \widehat{G}_{i} & \mapsto\left(\sum_{g \in G} x_{g} g\right) \widehat{G}_{i}
\end{aligned}
$$

A aplicação $\Phi$ esta bem definida. De fato, se consideramos $\alpha=\alpha_{1} \cdot\left(\frac{1+d}{2}\right)=\beta_{1} \cdot\left(\frac{1+d}{2}\right)=\beta$, onde $\alpha_{1}, \beta_{1} \in R^{\prime} A \widehat{G}_{i}$, temos $\alpha_{1}=\beta_{1}$ e, assim, $\Phi(\alpha)=\Phi(\beta)$.

Claramente $\Phi$ é um isomorfismo de anéis. Portanto,

$$
\left|R^{\prime} A\left(\frac{1+d}{2}\right) \widehat{G}_{i}\right|=\left|R^{\prime} G \widehat{G}_{i}\right|=\left|R^{\prime}\left(G / G_{i}\right)\right|=\left|R^{\prime}\right| \frac{|G|}{\left|G_{i}\right|}=\left|R^{\prime}\right|^{p^{i}} .
$$


Logo

$$
\left|R^{\prime} A\left(\frac{1+d}{2}\right) e_{i}\right|=\left|R^{\prime}\right|^{p^{i}-p^{i-1}}=\left(\frac{|R|}{\left|<a^{t-k}>\right|}\right)^{p^{i}-p^{i-1}}=\left(\frac{|\bar{R}|^{t}}{|\bar{R}|^{k}}\right)^{p^{i}-p^{i-1}}=|\bar{R}|^{(t-k)\left(p^{i}-p^{i-1}\right)} .
$$

Agora iremos calcular $\left|R A a^{k_{0}}\left(\frac{1+d}{2}\right) e_{0}\right|$. Por (4.7), temos

$$
\left|R A a^{k_{0}}\left(\frac{1+d}{2}\right) e_{0}\right|=\left|\left(\frac{R}{<a^{t-k_{0}}>}\right) A\left(\frac{1+d}{2}\right) e_{0}\right|
$$

Denotemos $\left(\frac{R}{<a^{t-k_{0}>}}\right)$ por $R^{\prime}$. Considere

$$
\begin{aligned}
\Phi: R^{\prime} A\left(\frac{1+d}{2}\right) \widehat{G} & \rightarrow R^{\prime} G \widehat{G} \\
\left(\sum_{g \in G} x_{g}\right)\left(\frac{1+d}{2}\right) \widehat{G} & \mapsto\left(\sum_{g \in G} x_{g}\right) \widehat{G}
\end{aligned}
$$

Claramente $\Phi$ é um isomorfismo entre os anéis $\quad R^{\prime} A\left(\frac{1+d}{2}\right) \widehat{G}$ e $\quad R^{\prime} G \widehat{G}$. Logo, $\left|R A a^{k_{0}}\left(\frac{1+d}{2}\right) e_{0}\right|=\left|\left(\frac{R}{<a^{t-k_{0}>}}\right) A\left(\frac{1+d}{2}\right) e_{0}\right|=\left|\left(R^{\prime} G\right) \widehat{G}\right|$. Como

$$
\left|R^{\prime} G \widehat{G}\right|=\left|R^{\prime}\right| \text { e }\left|R^{\prime}\right|=|\bar{R}|^{t-k_{0}}
$$

segue que $\left|<a^{k_{0}}\left(\frac{1+d}{2}\right) e_{0}>\right|=|\bar{R}|^{t-k_{0}}$.

Corolário 4.1.15 Se I é um ideal de $R G$ da forma

$I=<a^{k_{00}}\left(\frac{1+d}{2}\right) e_{0}>\oplus<a^{k_{01}}\left(\frac{1-d}{2}\right) e_{0}>\oplus \ldots \oplus<a^{k_{m 0}}\left(\frac{1+d}{2}\right) e_{m}>\oplus<a^{k_{m 1}}\left(\frac{1-d}{2}\right) e_{m}>$

então

$$
\left.|I|=|\bar{R}|^{[j=1} \sum^{m}\left(2 t-k_{j 0}-k_{j 1}\right)\left(p^{j}-p^{j-1}\right)+\left(2 t-k_{00}-k_{01}\right)\right] .
$$

Exemplo 4.1.16 Neste exemplo vamos considerar $p=3, n=2$ e $R=\mathbb{Z}_{25}$. Com isso, temos $G=<g_{0} ; g_{0}^{18}=1>e|\bar{R}|=5$. Como o(5) $=\phi(9)$ em $U\left(\mathbb{Z}_{18}\right)$, segue-se que 
$e_{i}=\left(\frac{1 \pm d}{2}\right) e_{i}$, com $e_{i}=\widehat{G}_{1}-\widehat{G}_{i-1}$, para $i=0,1,2$ são os idempotentes primitivos de $\mathbb{Z}_{25} G$, onde

$G_{i}=<g_{0}^{3^{i}}>$

Vamos analisar o peso de alguns códigos e o valor dos seus limitantes.

\begin{tabular}{|l|l|c|}
\hline Código & Peso & Limitante do Peso \\
$C_{1}=\left(\frac{1 \pm d}{2}\right) e_{1}$ & $4\left|G_{1}\right|=12$ & 17 \\
$C_{2}=\left(\frac{1 \pm d}{2}\right) e_{2}$ & $4\left|G_{2}\right|=4$ & 13 \\
$C_{3}=\left(\frac{1+d}{2}\right) e_{0} \oplus\left(\frac{1-d}{2}\right) e_{1}$ & $4\left|G_{1}\right|=12$ & 16 \\
$C_{4}=\left(\frac{1 \pm d}{2}\right) e_{0} \oplus\left(\frac{1 \pm d}{2}\right) e_{2}$ & $4\left|G_{2}\right|=4$ & 12 \\
$C_{5}=\left(\frac{1 \pm d}{2}\right) e_{1} \oplus\left(\frac{1 \pm d}{2}\right) e_{2}$ & $4\left|g_{2}\right|=4$ & 11 \\
\hline
\end{tabular}

\subsection{Códigos Livres de Comprimento $2 p^{n}$ Sobre Anéis} de Cadeia

Nesta seção caracterizaremos códigos livres de comprimento $2 p^{n}$ sobre anéis de cadeia. Isto será feito de modo análogo aos códigos livres de comprimento $p^{n}$ sobre anéis de cadeia. Iremos considerar $A$ um grupo cíclico de ordem $2 p^{n}, R$ anel de cadeia com $m d c(|R|,|A|)=1$ e os idempotentes primitivos ortogonais $\left(\frac{1 \pm d}{2}\right) e_{i}, \operatorname{com} 0 \leq i \leq n$.

Teorema 4.2.1 Seja $\gamma$ um transversal de $G_{i-1}$ em $G$ e $\tau$ um transversal de $G_{i}$ em $G_{i-1}$. Então $R A\left(\frac{1 \pm d}{2}\right) e_{i}$ é um código livre com base

$$
\mathcal{B}=\left\{c(1-b)\left(\frac{1 \pm d}{2}\right) \widehat{G}_{i} \mid c \in \gamma, b \in \tau \backslash\{1\}\right\}
$$

sobre $R$, onde o sinal positivo nos elementos da base é referente ao ideal $R A\left(\frac{1+d}{2}\right) e_{i}$ e o sinal negativo é referente ao ideal $R A\left(\frac{1-d}{2}\right) e_{i}$. 
Prova: Faremos a demonstração de que o código $R A\left(\frac{1+d}{2}\right) e_{i}$ é livre. A demonstração de que $R A\left(\frac{1-d}{2}\right) e_{i}$ é livre é análoga. Primeiramente vamos provar que os elementos de $\mathcal{B}$ pertencem ao código.

1) Para $b \in \tau \backslash\{1\}$, temos $(1-b)\left(\frac{1+d}{2}\right) \widehat{G}_{i-1}=\left(\frac{1+d}{2}\right) \widehat{G}_{i-1}-\left(\frac{1+d}{2}\right) b \widehat{G}_{i-1}=0$, pois $b \cdot \widehat{G}_{i-1}=\widehat{G}_{i-1}$.

2) $c(1-b)\left(\frac{1+d}{2}\right) \widehat{G}_{i}=c(1-b)\left(\frac{1+d}{2}\right)\left(\widehat{G}_{i}-\widehat{G}_{i-1}+\widehat{G}_{i-1}\right)=c(1-b)\left(\frac{1+d}{2}\right) e_{i}+c(1-b)\left(\frac{1+d}{2}\right) \widehat{G}_{i-1}=$ $c(1-b)\left(\frac{1+d}{2}\right) e_{i}$. Portanto, $\mathcal{B} \subset R A\left(\frac{1+d}{2}\right) e_{i}$.

Agora, mostraremos que os elementos de $\mathcal{B}$ são linearmente independentes.

Sejam $x_{c b} \in R$, onde $c \in \gamma$ e $b \in \tau \backslash\{1\}$ são tais que

$$
\sum_{c \in \gamma} x_{b \in \tau \backslash\{1\}} c(1-b)\left(\frac{1+d}{2}\right) \widehat{G}_{i}=0 .
$$

Desenvolvendo a igualdade acima temos

$$
\begin{aligned}
0 & =\sum_{c \in \gamma}\left(\sum_{b \in \tau} x_{c b} c(1-b)\left(\frac{1+d}{2}\right) \widehat{G}_{i}\right) \\
& =\sum_{c \in \gamma}\left(\sum_{b \in \tau} x_{c b} c\left(\frac{1+d}{2}\right) \widehat{G}_{i}\right)-\sum_{c \in \gamma} x_{c b \tau} c b\left(\frac{1+d}{2}\right) \widehat{G}_{i} \\
& =\frac{1}{2}\left(\sum_{c \in \gamma}\left(\sum_{b \in \tau} x_{c b} c \widehat{G}_{i}\right)-\sum_{c \in \gamma} x_{c b} c b \widehat{G}_{i}\right)+\frac{1}{2}\left(\sum_{c \in \gamma}\left(\sum_{b \in \tau} x_{c b} d c \widehat{G}_{i}\right)-\sum_{c \in \gamma} x_{c b} d c b \widehat{G}_{i}\right) .
\end{aligned}
$$

O que faremos agora será analisar cada elemento deste somatório. É claro que os elementos do somatório $\left(\sum_{c \in \gamma}\left(\sum_{b \in \tau} x_{c b} c \widehat{G}_{i}\right)-\sum_{c \in \gamma} x_{b \in \tau} c b \widehat{G}_{i}\right)$ têm suporte disjunto com os elementos do somatório $\left(\sum_{c \in \gamma}\left(\sum_{b \in \tau} x_{c b} d c \widehat{G}_{i}\right)-\sum_{c \in \gamma} x_{b \in \tau} d c b \widehat{G}_{i}\right)$. Assim,

$$
\left(\sum_{c \in \gamma}\left(\sum_{b \in \tau} x_{c b} c \widehat{G}_{i}\right)-\sum_{c \in \gamma} x_{b \in \tau} c b \widehat{G}_{i}\right)=\left(\sum_{c \in \gamma}\left(\sum_{b \in \tau} x_{c b} d c \widehat{G}_{i}\right)-\sum_{c \in \gamma} x_{b \in \tau} d c b \widehat{G}_{i}\right)=0 .
$$

Provaremos que os elementos do somatório $\left(\sum_{c \in \gamma}\left(\sum_{b \in \tau} x_{c b} c \widehat{G}_{i}\right)-\sum_{c \in \gamma} x_{b \in} c b \widehat{G}_{i}\right)$ têm suporte disjunto. Para isso, provaremos que, para cada $c, b$ fixos, o elemento $c b \widehat{G}_{i}$ tem suporte 
disjunto de qualquer outro elemento nesta combinação linear. Como $b \in \tau$, então $\widehat{G}_{i}$ e $b \widehat{G}_{i}$ têm suportes disjuntos. Logo $c \widehat{G}_{i}$ e $c b \widehat{G}_{i}$ têm suporte disjunto. É claro que se $c_{j} \neq c_{k}$, então $c_{j} \widehat{G}_{i}$ e $c_{k} \widehat{G}_{i}$ têm suportes disjuntos. Como $\tau$ é um transversal de $G_{i}$ em $G_{i-1}$, temos que para $b_{j} \neq b_{k} \in \tau, c_{j} b_{j} \widehat{G}_{i}$ e $c_{k} b_{k} \widehat{G}_{i}$ têm suportes disjuntos. Portanto, $x_{c b}=0, \forall c \in \gamma$ e $b \in \tau$.

Pelo Teorema 4.1.14, o número de elementos do código $R A\left(\frac{1+d}{2}\right) e_{i}$ é dado por

$$
|\bar{R}|^{t\left(p^{i}-p^{i-1}\right)}=|R|^{\left(p^{i}-p^{i-1}\right)} .
$$

O número de elementos do código gerado por $\mathcal{B}$ sobre $R$ é dado por $|R|^{(|\gamma| \cdot(|\tau|-1))}$.

$$
(|\gamma| \cdot(|\tau|-1))=\frac{|G|}{\left|G_{i-1}\right|} \cdot\left(\frac{\left|G_{i-1}\right|}{\left|G_{i}\right|}-1\right)=\left(p^{i}-p^{i-1}\right) .
$$

Como o código gerado por $\mathcal{B}$ está contido em $R A\left(\frac{1+d}{2}\right) e_{i}$ e possuem o mesmo número de elementos, eles são iguais. Assim $R A\left(\frac{1+d}{2}\right) e_{i}$ é um código livre com base $\mathcal{B}$.

É claro que se o código $C$ é da forma $C=R A a^{k}\left(\frac{1+d}{2}\right) e_{i}$, onde $0<k<t$, então $C$ não é livre, pois $\alpha \cdot a^{t-k} \cdot a^{k}\left(\frac{1+d}{2}\right) e_{i}=0$, para todo $\alpha \in R A$. 


\section{CAPÍTULO 5}

\section{Conclusões Finais}

Em [10], Dinh e López-Permouth caracterizaram todos os códigos cíclicos de comprimento $n$ sobre anéis de cadeia $R$, onde $|R|=q^{k}$ e $q \nmid n$ da seguinte forma:

Seja $x^{n}-1=f_{0} \ldots f_{m}$ a representação de $x^{n}-1$ como produto de polinômios básico irre-

dutíveis coprimos dois a dois. Então qualquer ideal em $\frac{R[x]}{\left\langle x^{n}-1\right\rangle}$ é da forma $\left\langle a^{j} \widehat{f}_{i}+\left\langle x^{n}-1\right\rangle\right\rangle$, onde $0 \leq j \leq t$ e $0 \leq i \leq m$.

A seguir, vamos enunciar um teorema que foi muito utilizado no artigo [10].

Teorema 5.0.2 (Teorema 3.4) Seja C um código cíclicos de comprimento n sobre um anel de cadeia finito $R$ ( $R$ têm ideal maximal $\langle a\rangle$, com $t$ o índice de nilpotência de a). Então existe uma única família de polinômios $F_{0}, F_{1}, \ldots, F_{t} \in R[x]$, mônicos e coprimos dois a dois tais que $F_{0} F_{1} \ldots F_{t}=x^{n}-1$ e $C=\left\langle\widehat{F}_{1}, a \widehat{F}_{2}, \ldots, a^{t-1} \widehat{F}_{t}\right\rangle$.

Para demonstrar tal teorema, Dinh e López-Permouth assumiram que poderiam escrever o código $C$ da seguinte forma: 


$$
\begin{aligned}
C= & <\widehat{f}_{k_{1}+1}>\oplus \ldots \oplus<\widehat{f}_{k_{1}+k_{2}}>\oplus<a \widehat{f}_{k_{1}+k_{2}+1}>\oplus \ldots \oplus<a \widehat{f}_{k_{1}+k_{2}+k_{3}}>\oplus \ldots \oplus \\
& \oplus<a^{t-1} \widehat{f}_{k_{1}+\ldots+k_{t}+1}>\oplus \ldots \oplus<a^{t-1} \widehat{f}_{m}>
\end{aligned}
$$

onde $k_{1}, \ldots, k_{t} \geq 0$ e $k_{1}+\ldots+k_{t}+1 \leq m$.

Depois, foram definidos os polinômios $F_{i}^{\prime} s$ da seguinte forma:

Sejam $k_{0}=0$ e $k_{t+1}$ um inteiro não negativo, tais que $k_{1}+\ldots+k_{t+1}=m$. Para $i=0, \ldots, t$ defina

$$
F_{i}=f_{k_{0}+\ldots+k_{i}+1} \ldots f_{k_{0}+\ldots+k_{i+1}} .
$$

Observe porém que um código cíclico qualquer não necessariamente é da forma que foi descrito em 5.1. Por exemplo, $C=<\widehat{f}_{k_{1}+1}>$ (mesma notação que foi utilizada em 5.1) é um código cíclico e desta forma não existiriam $F_{0}, \ldots, F_{t}$ funções como foi enunciado no teorema. Outro caso em que o código não poderia ser descrito como no Teorema 5.0.2 é quando $t>m$.

Quando provamos todos os resultados de Dinh e López-Permouth [10] referentes à códigos cíclicos, duais e auto-duais, corrigimos este erro dito acima e, além disso, através da abordagem via anéis de grupo, foi possível demonstrar todos os resultados de uma forma mais simples e direta. Conseguimos também resultados novos (Capítulo 3 e 4) que são de grande relevância para a Teoria dos Códigos Corretores de Erros, que são os cálculos dos pesos mínimos de todos os códigos cíclicos sobre anéis de cadeia de comprimento $p^{n}$ e $2 p^{n}$ e também a caracterização dos códigos cíclicos livres de comprimento $p^{n}$ e $2 p^{n}$, sendo que quando o comprimento do código é $p^{n}$ estamos sob as condições da hipótese A e quando o comprimento do código é $2 p^{n}$ estamos sob as condições da hipótese B. Exibimos também bases para ideais que são somando direto de códigos livres.

Neste trabalho, abordamos dois assuntos dentre os três assuntos principais da Teoria dos Códigos Corretores de Erros que são:

- Caracterização de códigos; 
- Calculo dos pesos minimais;

- Descrição de algoritmos de decodificação.

Como perspectivas futuras, pretendemos desenvolver um algoritmo de decodificação para códigos cíclicos sobre anéis de cadeia, caracterizar e calcular os pesos mínimos de códigos cíclicos sobre anéis seriais e obter resultados sobre códigos cíclicos duais, auto-ortogonais e auto-duais sobre anéis seriais. 


\section{Referências Bibliográficas}

[1] M. F. Atiyah, I. G. Macdonald, Introduction to Commutative Algebra, Westview Press, Massachusetts, 1969. 7

[2] N. S. Babu, K. H. Zimmermann, Decoding of linear codes over Galois rings, IEEE Transactions on Informations Theory, Vol 47 (2001) 1599-1603. 4

[3] I. F. Blake, R. C. Mullin, The Mathematical Theory of Coding, Academic Press, Inc, New York, 1975.

[4] D. M. Burton, Elementary Number Theory, Series in Pure and Applied Mathematics, 4th ed, New York, 1998. 19

[5] A. R. Calderbank, A. R. Hammons, Jr., P. V. Kumar, N. J. A. Sloane, P. Solé, A linear construction for certain Kerdock and Preparata codes, Bull. Amer. Math., Vol 29 (1993) 218-222. 3

[6] A. R. Calderbank, N. J. A. Sloane, Modular and p-adic codes, Designs, Codes and Cryptography, Vol 6 (1995) 21-35. 3, 4, 13

[7] A. C. A. Campello Jr., G. C. Jorge, S. I. R. Costa, Decoding q-ary lattices in the Lee metric, IEEE Information Theory Workshop, (2011) 220-224. 4

[8] J. H. Conway, N. J. A. Sloane, Self-dual codes over the integers modulo 4, J. Combin. Theory Ser. A, Vol 62 (1993) 30-45. 3

[9] H. Q. Dinh, Complete distances of all negacyclic codes of length $2^{s}$ over $\mathbb{Z}_{2^{a}}$, IEEE transaction on Information Theory, Vol 53 (2007) 147-161. 3 
[10] H. Q. Dinh, S. R. López-Permouth, Cyclic and negacyclic codes over finite chain rings, IEEE Transactions on Information Theory, Vol. 50 (2004) 1728-1744. 4, 23, 32, 85, 86

[11] S. T. Dougherty, J. Kim, H. Liu, Construction of self-dual codes over finite commutative chain rings, Int. Journal on Information and Coding Theory, (2010). 3

[12] Y. A. Drodz, V. V. Kirichenko, Finite Dimensional Algebras, Springer-Verlag, Berlin, 1991.

[13] F. Dutra, R. Ferraz, C. Polcino Milies, Semisimple group codes and dihedral codes, Algebra and Discrete Mathematics, Vol 3 (2009) 28-48. 46

[14] A. Hefez, M. L. T. Villela, Códigos Corretores de Erros, IMPA, Série de Computação e Matemática, Rio de Janeiro, 2008. 15

[15] R.A. Ferraz, C. Polcino Milies; Idempotents in group algebras and minimal abelian codes, ScienceDirect, Finite Fields and their Applications, Vol 13 (2007) 382-393. 37, 38, 58

[16] A. R. Hammons Jr., P.V. Kumar, A.R.Calderbank, N.J.A. Sloane, P. Solé, The $Z_{4}$-linearity of Kerdock, Preparata, Goethals, and related codes, IEEE Transactions on Information Theory, Vol 40 (1994) 301319. 3

[17] W. C. Huffman, V. Pless, Fundamentals of Error-Correcting Codes, Cambridge University Press, Cambridge, 2003. 3,13

[18] N. Jacobson, Basic Algebra, W.H.Freeman and Company, San Francisco, 1980. 7, 9, 45, 47

[19] P. Kanwar, S. R. López-Permouth, Cyclic codes over the interger modulo $p^{m}$, Finite Fields Appl. Vol 3 (1997) 334-352. 3, 4

[20] Z. H. Liu, Notes on linear codes over finite chain rings, Acta Mathematicae Applicatae Sinica, Vol 27 (2011) 141-148. 3

[21] B. R. MacDonald, Finite Rings With Identity, Pure and Applied Mathematics, A Series of Monographs and Textbooks, New York, 1974. 7, 10, 12

[22] F. J. MacWilliams, N. J. A. Sloane The Theory of Error-Correcting Codes, North-Holland Publishing Company, New York, 1977. 18

[23] F. Melo, Sobre Códigos Cíclicos e Abelianos, Tese de doutorado a ser apresentada ao Instituto de Matemática e Estatística, USP, São Paulo, 2012. 40 
[24] F. J. MacWilliams, N. J. A. Sloane, The Theory of Error-Correcting Codes, The Netherlands:NorthHolland, Amsterdam, 1998. 16

[25] E. Martínez-Moro, I. F. Rúa, On repeated-root multivariable codes over a finite chain ring, Des. Codes Cryptogr., Vol 45 (2007) 219-227. 3

[26] H. Matsumura, Commutative Ring Theory, Cambridge University Press, New York, 1986. 7

[27] I. Niven, H. S. Zuckerman and H. L. Montgomery, An Introduction to the Theory of numbers, John Wiley and Sons.Inc, New York, 1991. 19

[28] G. Norton, A. Sălăgean-Mandache, On the structure of linear cyclic codes over finite chain rings, Appl. Algebra Eng. Commun. Comput., Vol 10 (2000) 489-506. 3, 4

[29] G. T .Peterson, Lifting idempotents in near rings; Arch. Math., Vol 51 (1988) 208-212.

[30] V. Pless, Z. Qian, Cyclic codes and quadratic residue codes over $Z_{4}$, IEEE Transactions on Information Theory, Vol. 42 (1996) 1594-1600. 3, 13

[31] C. Polcino Milies, S. K. Sehgal, An introduction to Group Rings, Kluwer Academic Publishers, Dordrecht, Netherlands, 2002. 7

[32] G. Puninski, Serial Rings; Kluwer Academic Publishers, Dordrecht, Boston, London, 2001.

[33] D. Redmond, Number Theory: An Introduction, ser. Monographs and Textbooks in Pure and Applied Mathematics. New York, 1996. 19

[34] S. Roman, Coding and Information Theory, Kluwer Academic Publishers, Graduate Texts in Mathematics, Springer, New York, 1992. 16, 18

[35] J. P. O. Santos, Introdução à Teoria dos Números, Associoação Instituto Nacional de Matemática Pura e Aplicada, Rio de Janeiro, 2003. 19

[36] C. Vanden Eynden, Elementary Number Theory, McGraw-Hill, 2nd ed. New York, 2001. 19

[37] Z. Wan, cyclic codes over Galois rings, Alg. Colloq., Vol. 6 (1999) 291-304. 3 